\title{
Vaccine-induced protective immunity against \\ Coxiella burnetii
}

\author{
A Dissertation \\ presented to \\ the Faculty of the Graduate School \\ at the University of Missouri-Columbia
}

In Partial Fulfillment

of the Requirements for the Degree

Doctor of Philosophy

by

LINDSEY ELIZABETH LEDBETTER

Dr. Guoquan Zhang, Dissertation Advisor

December 2019 
The undersigned, appointed by the dean of the Graduate School, have examined the dissertation entitled

\section{Vaccine-induced protective immunity against Coxiella burnetii}

presented by Lindsey E. Ledbetter,

a candidate for the degree of Doctor of Philosophy,

and hereby certify that, in their opinion, it is worthy of acceptance.

Professor Guoquan Zhang

Professor Charles Brown

Professor Jerod Skyberg

Professor Jeffrey Adamovicz

Professor Mark Daniels 


\section{DEDICATION}

I dedicate this work to my son, Jameson Noble, who motivates me to be the best that I can be. To my husband, the love of my life, who makes life fun and has been a constant source of smiles and laughs during this difficult journey. And to my parents, who have been my cheerleaders through life, inspiring me to persevere. 


\section{ACKNOWLEDGEMENTS}

This work would not have been possible without support from my incredible dissertation committee. l'd first like to thank Drs. Charles Brown and Jerod Skyberg for the time they spent mentoring me, discussing my data, proofreading my manuscripts, and ultimately teaching me how to think like a scientist. Additionally, I'd like to thank Dr. Jeffrey Adamovicz for imparting his knowledge of vaccine and therapeutic design to me. l'd also like to thank Dr. Mark Daniels for lending his expertise in T cell biology and for inspiring me to think outside the box.

Thank you to my dissertation advisor, Dr. Guoquan Zhang, for giving me a lab home and for providing the space for me to explore the mechanisms of vaccine immunity detailed here. Also, thank you to Dr. Rama Cherla for helping me with the Coxiella-specific ELISAs and for sacrificing your time to assist me in the ABSL3. I'd also like to thank Dr. Laura Buttrum for training me when I joined the lab.

My peers know how difficult graduate school can be, and l'd like to thank them for being a constant source of support over the last 5 years. l'd especially like to thank my friends in Connaway Hall who were always there to talk when I needed it. Special thanks to Dr. Katie Chambers and the Comparative Medicine residents for helping me in the ABSL-3 and to Alexis Dadelahi for proofreading my dissertation.

I'd like to thank the University of Missouri Laboratory for Infectious Disease Research (MU-LIDR) for providing the facilities to do this work. l'd especially like to thank Drs. Paul Anderson and Jeffrey Whyte for their time and assistance in the 
Aerobiology and Immunology cores at the MU-LIDR. A special thank you to Dr. Travis McCarthy who trained me on biocontainment procedures, making me a better scientist overall. The bulk of this work involved animals and would, therefore, not have been possible without help from the animal care staff. l'd especially like to thank Raye Lynn Allen and Jennifer Blair for facilitating my work in the BSL-2 and ABSL-3 labs. Thank you to Daniel Jackson and the Cell and Immunobiology core $(\mathrm{ClC})$ for training me and assisting with my flow cytometry experiments. Also, thank you to Dr. William J. Mitchell for scoring my histology slides.

l'd like to thank my collaborators who provided guidance and reagents to support this work including Dr. Ryan Metzler with Miltenyi Biotec, who helped with my initial immunophenotype analysis on the MACSQuant Analyzer 10 and provided all of the necessary reagents. Additional thanks to Dr. Elizabeth Jacobsen at Mayo Clinic for lending her expertise in eosinophil biology and to Dr. Robyn Klein at Washington University for providing the IFN- $\gamma$ knockout mice.

Finally, l'd like to thank Kim Sysoukrath, my former supervisor at Tyson Foods, for working alongside me and patiently training me on proper aseptic technique. This basic skill set me up for success as a scientist before I ever knew this was a career l'd be pursuing. I'd also like to thank Dr. Billy Hargis, my former Poultry Diseases instructor, for getting me excited about research as a career and for inspiring me to go to graduate school. And thank you to Dr. Daniel Voth, my undergraduate honors thesis mentor, who fostered my love for Coxiella and gave me the tools to succeed in graduate school and beyond. 


\section{TABLE OF CONTENTS}

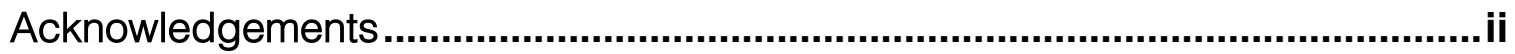

List of Figures .............................................................................................ii

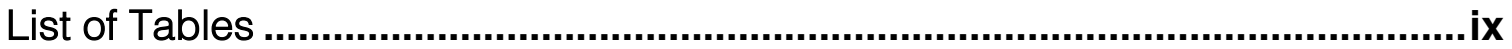

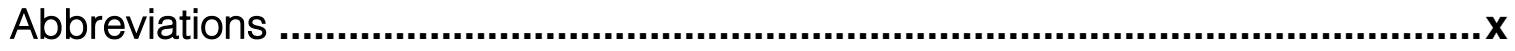

Abstract

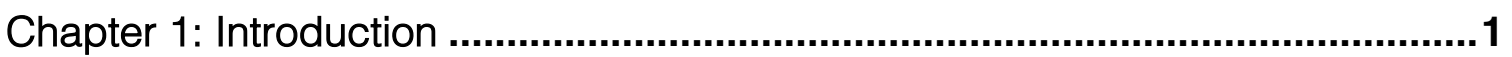

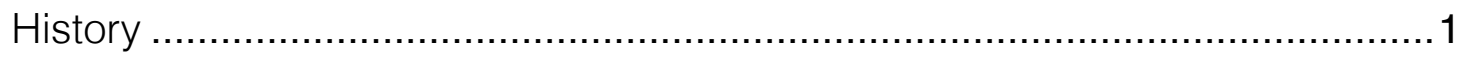

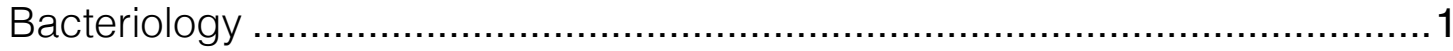

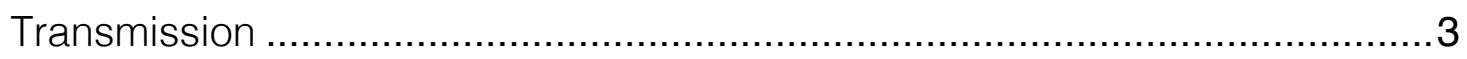

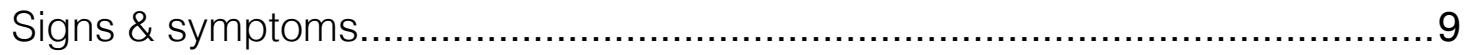

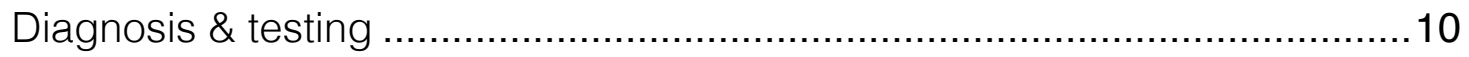

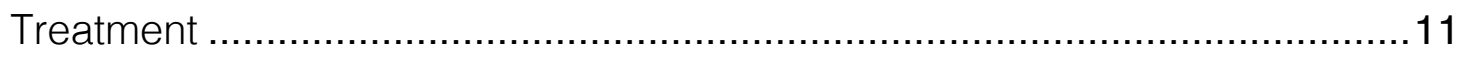

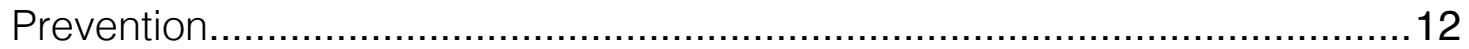

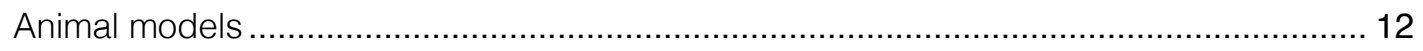

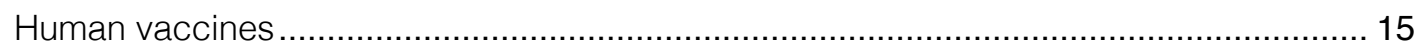

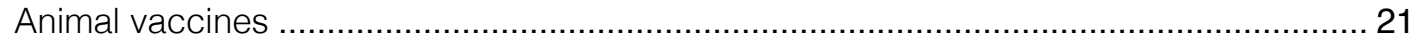

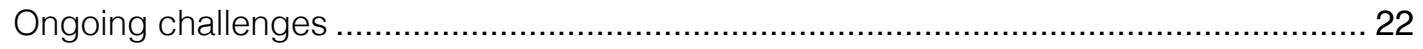

Immunological mechanisms of vaccination ............................................................... 23

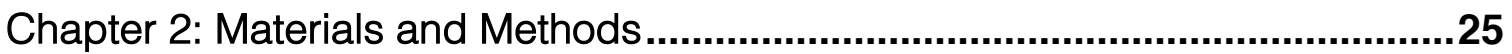

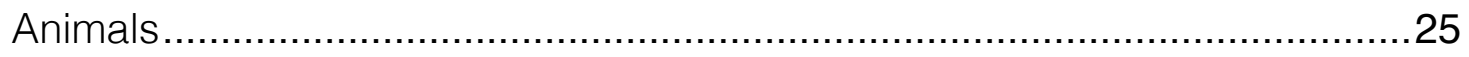

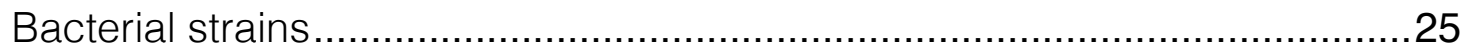


Vaccination.

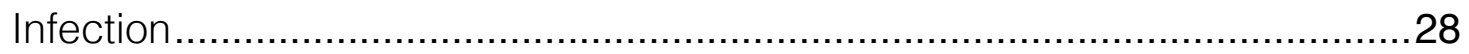

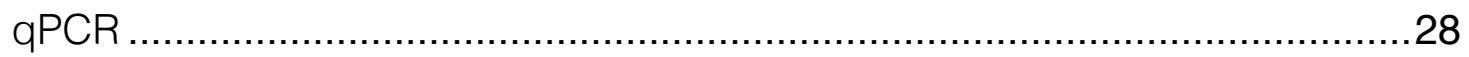

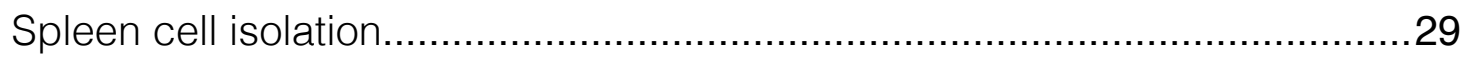

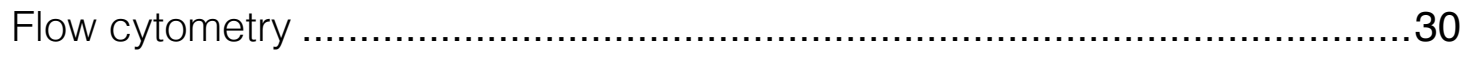

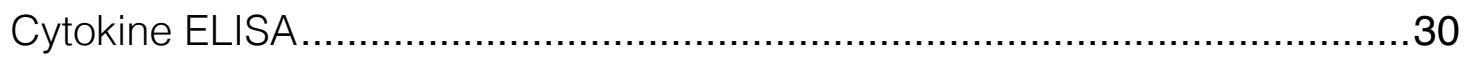

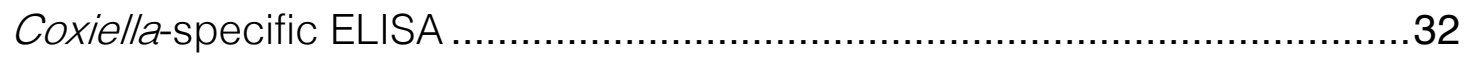

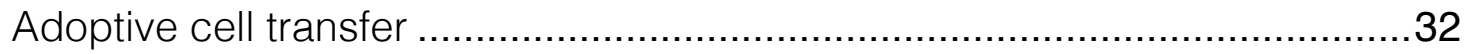

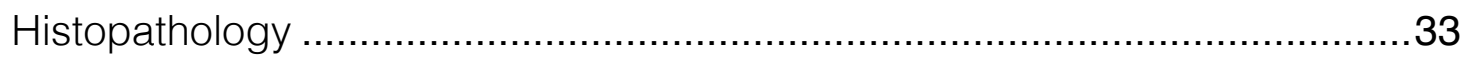

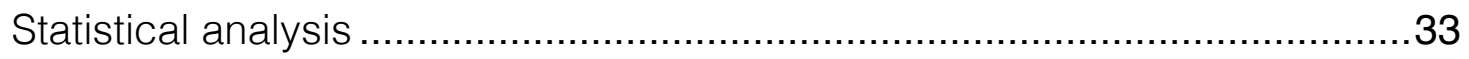

\section{Chapter 3: Eosinophils affect antibody isotype switching and may partially} contribute to early vaccine-induced immunity against Coxiella burnetii ............34

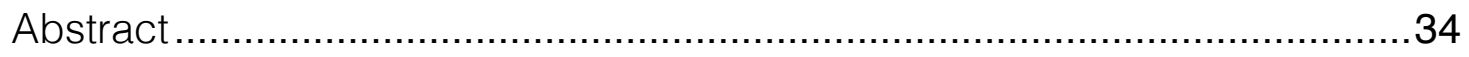

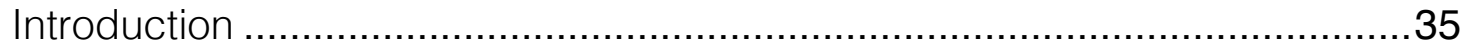

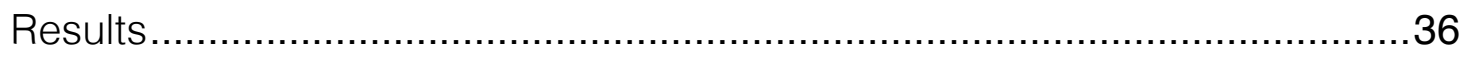

Host cell-free $C$. burnetï retains immunogenicity and protectivity .................................... 36

PIV protects BALB/c mice as early as 1 week post-vaccination ..................................... 37

$\mathrm{SSC}^{\text {high }}$ granulocytes express CD11b and the interleukin-5 receptor ............................. 41

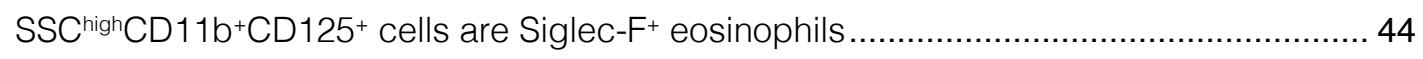

Eosinophil accumulation in PIV-vaccinated mice is $\mathrm{CD}^{+}{ }^{+} \mathrm{T}$ cell-dependent ...................... 44

Eosinophils may partially contribute to early PIV protection .............................................. 46

Eosinophils contribute to antibody isotype switching in late PIV responses....................... 48

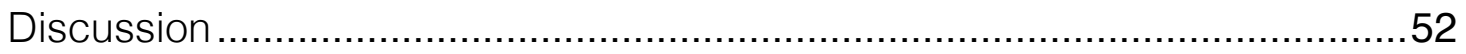


Chapter 4: MHC-II-restricted, CD4+ ${ }^{+}$cell-dependent and -independent mechanisms are required for vaccine-induced protective immunity against

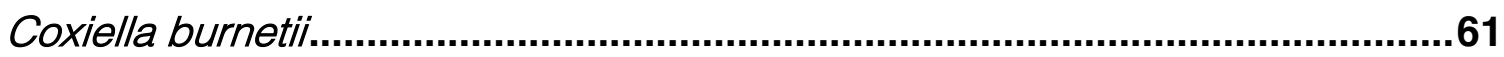

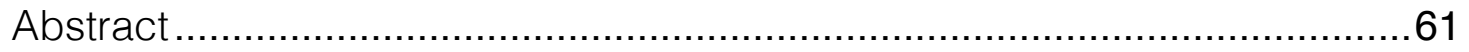

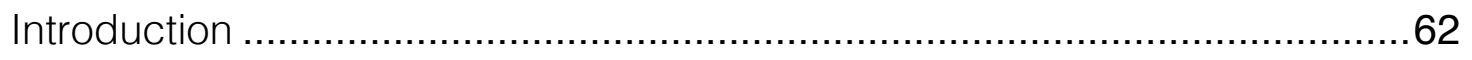

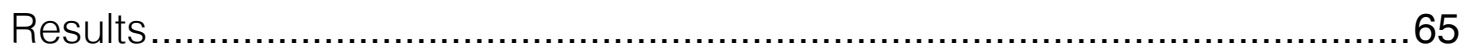

MHC-II is important for PIV-mediated protection against C. burnetii .................................. 65

MHC-II-dependent vaccine protection partially depends on CD4+ $\mathrm{T}$ cells......................... 67

CD4 ${ }^{+}$T cells from PIV-vaccinated mice are sufficient to generate protection ………......... 70

Tbet plays a role in PIV protection that partially depends on Th1 CD4+ T cells................. 76

IFN- $\gamma$ modulates inflammation but may not be involved in bacterial clearance .................. 78

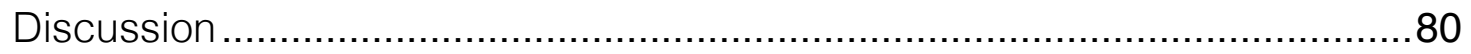

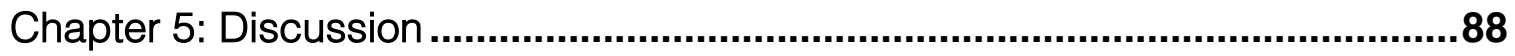

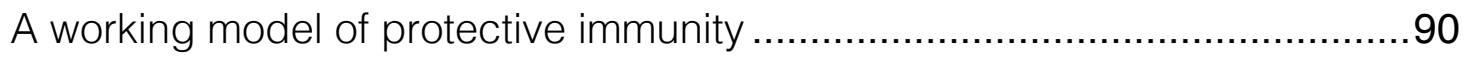

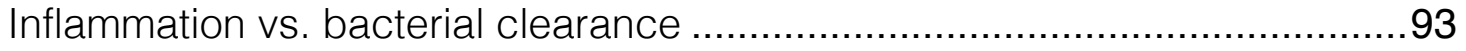

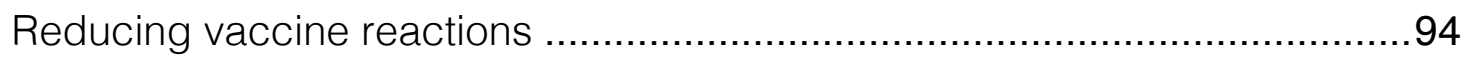

Boosting innate immunity through adjuvant selection ..................................96

Targeting T cells with epitope selection..................................................98

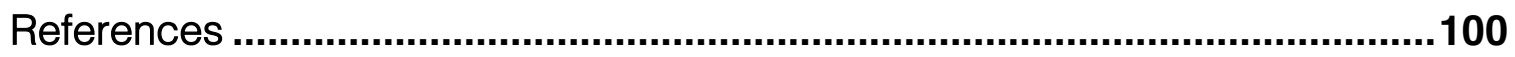

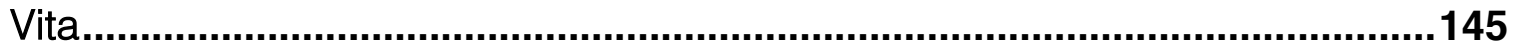




\section{Footnotes:}

A. Chapter 3. This work was published in Infection and Immunity in 2019 with Lindsey Ledbetter as the first author, followed in authorship order by Rama Cherla, Catherine Chambers, Yan Zhang, and Guoquan Zhang. LL conceived the project, designed and executed experiments, analyzed data, and wrote the paper. RC assisted with Coxiella-specific ELISAs. CC assisted with necropsy in ABSL-3 experiments. $Y Z$ supported data analysis. GZ conceived the project and edited the manuscript.

B. Chapter 4. This work was published in Infection and Immunity in 2019 with Lindsey Ledbetter as the first author, followed in authorship order by Rama Cherla, Catherine Chambers, Yan Zhang, William J. Mitchell, and Guoquan Zhang. LL conceived the project, designed and executed experiments, analyzed data, and wrote the paper. RC assisted with Coxiella-specific ELISAs. CC assisted with necropsy in ABSL-3 experiments. YZ supported data analysis. WJM scored histopathology. GZ conceived the project and designed experiments. 


\section{LIST OF FIGURES}

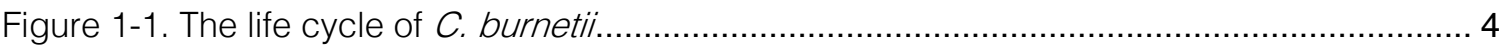

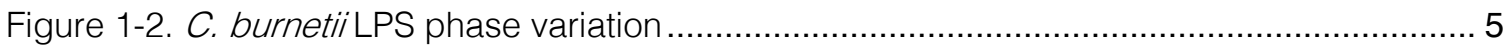

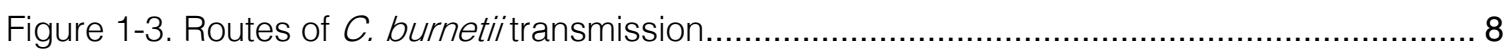

Figure 3-1. Host cell-free $C$. burnetii retains immunogenicity and protectivity........................... 38

Figure 3-2. PIV protects BALB/c mice as early as 1 week post-vaccination.............................. 39

Figure 3-3. PIV significantly increases high side scatter granulocytes in the spleen .................. 42

Figure 3-4. SSC ${ }^{\text {high }}$ granulocytes express CD11b and the interleukin-5 receptor .......................43

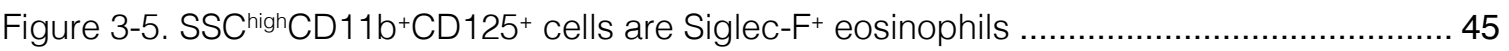

Figure 3-6. Eosinophil accumulation in PIV-vaccinated mice is CD4+ $4^{+}$cell dependent............. 47

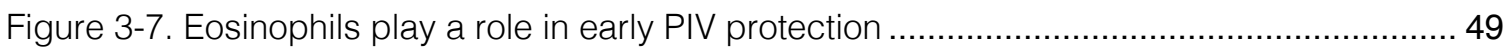

Figure 3-8. Eosinophils contribute to antibody isotype switching in late PIV responses ............. 51

Figure 3-9. A working model for eosinophils in PIV-mediated protection .................................. 60

Figure 4-1. MHC-II is important for PIV-mediated protection against $C$. burnetii........................ 68

Figure 4-2. MHC-I and MHC-II are involved in antibody isotype switching ............................... 69

Figure 4-3. MHC-II-dependent vaccine protection is partially dependent on CD4+ T cells......... 71

Figure 4-4. CD4+ T cells are important for antibody isotype switching ..................................... 72

Figure 4-5. CD4+ T cells from PIV-vaccinated mice are sufficient to generate protection ........... 74

Figure 4-6. CD4+ T cells from naïve mice are detrimental to protection..................................... 75

Figure 4-7. Tbet plays a role in vaccine protection that partially depends on Th1 CD4+ $T$ cells . 77

Figure 4-8. IFN- $\gamma$ modulates inflammation but may not be involved in bacterial clearance......... 79

Figure 5-1. A systems vaccinology approach for rational vaccine design ............................... 89

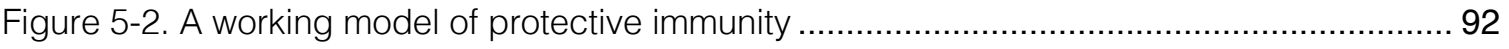




\section{LIST OF TABLES}

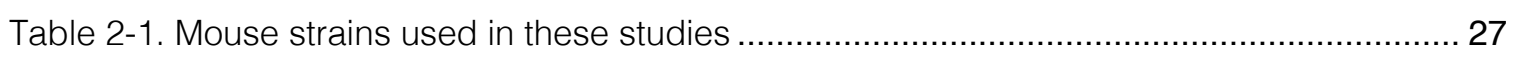

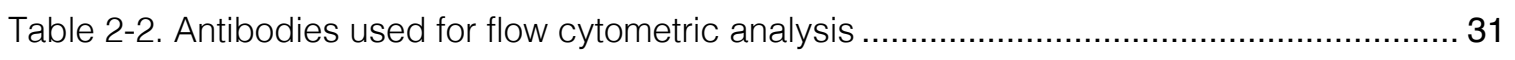




\section{ABBREVIATIONS}

A ABSL-3 animal biosafety level 3

ACCM-2 acidified citrate cysteine medium-2

ACCM-D defined acidified citrate cysteine medium

APC antigen-presenting cell

B B2m beta-2-microglobulin

BSL-3 biosafety level 3

C CCV Coxiella-containing vacuole

CMI cell-mediated immunity

CMR chloroform:methanol residue

D DC dendritic cell

dpi days post-infection

dpv days post-vaccination

DTH delayed-type hypersensitivity

E EDN eosinophil-derived neurotoxin

ELISA enzyme-linked immunosorbent assay

EMA European Medicines Agency

F FACS fluorescence-activated cell sorting

FcRn neonatal Fc receptor

FSC forward scatter

H HLA human leukocyte antigen 


\begin{tabular}{|c|c|c|}
\hline \multirow[t]{5}{*}{ I } & i.d. & intradermal \\
\hline & i.n. & intranasal \\
\hline & i.p. & intraperitoneal \\
\hline & i.t. & intratracheal \\
\hline & IFN- $\gamma$ & interferon gamma \\
\hline \multirow[t]{2}{*}{$\mathbf{K}$} & $\mathrm{Kd}$ & kilodalton \\
\hline & $\mathrm{KO}$ & knockout \\
\hline \multirow[t]{2}{*}{ L } & LCV & large cell variant \\
\hline & LPS & lipopolysaccharide \\
\hline \multirow[t]{4}{*}{ M } & MBP & major basic protein \\
\hline & MHC-I & major histocompatibility complex class I \\
\hline & MHC-II & major histocompatibility complex class II \\
\hline & MPL & monophosphoryl lipid A \\
\hline \multirow[t]{3}{*}{$\mathbf{N}$} & NK cell & natural killer cell \\
\hline & NMI & C. burnetii Nine Mile phase I \\
\hline & NMII & C. burnetii Nine Mile phase II \\
\hline \multirow[t]{5}{*}{$\mathbf{P}$} & PAMP & pathogen-associated molecular pattern \\
\hline & PBMC & peripheral blood mononuclear cell \\
\hline & PBS & phosphate-buffered saline \\
\hline & PCR & polymerase chain reaction \\
\hline & PIV & formalin-inactivated $C$. burnetii Nine Mile phase I vaccine \\
\hline
\end{tabular}


PIIV formalin-inactivated C. burnetii Nine Mile phase II vaccine

PRR pattern recognition receptor

PV parasitophorous vacuole

Q QFS post-Q fever fatigue syndrome

qPCR real-time quantitative PCR

S s.c. subcutaneous

SCID severe combined immunodeficient

SCV small cell variant

SPF specific pathogen-free

SSC side scatter

T TCA trichloroacetic acid

TLR Toll-like receptor

TNF- $\alpha \quad$ tumor necrosis factor alpha

W WT wild-type 


\section{ABSTRACT}

Coxiella burnetii is an obligate intracellular Gram-negative bacterial pathogen and the causative agent of human $Q$ fever. This disease presents acutely as a flu-like illness, although it can escalate to a chronic and often fatal disease when left untreated. Considering no FDA-approved vaccine exists, the creation of a safe and effective vaccine remains an important public health goal. A formalin-inactivated C. burnetii Nine Mile strain phase I whole-cell vaccine generates protective immunity in a mouse model of experimental $Q$ fever, although the mechanisms of protection remain unclear. Chapter 3 details my work establishing an early vaccine protection model and elucidating the cellular immune response which elicits early protection. The early time point at which PIV protects has implications for its use as a therapeutic vaccine. Furthermore, the innate-driven mechanisms by which it protects can be exploited for an improved $Q$ fever vaccine. The importance of $T$ cells in vaccine immunity against $C$. burnetii is well supported, however, multiple questions remain. It is unclear how $\mathrm{CD}^{+}$or $\mathrm{CD} 8^{+} \mathrm{T}$ cells contribute to vaccine protection, and the role of specific CD4+ $\mathrm{T}$ cell subsets is unknown. IFN- $\gamma$ is critical for primary defense against $C$. burnetii, though its importance in vaccine immunity is undetermined. Chapter 4 describes my work aimed at filling these knowledge gaps. Vaccine development efforts have largely focused on the generation of antibodies as a correlate of protection. It has become clear that targeting T cells is more critical to vaccine protection and a better understanding of the mechanisms of cell-mediated immunity will inform future $Q$ fever vaccine development. 


\section{CHAPTER 1: INTRODUCTION}

\section{History}

Coxiella burnetii is an obligate intracellular Gram-negative bacterial pathogen and the causative agent of the zoonosis human $Q$ fever. It was discovered in 1935, when E. H. Derrick was tasked with identifying a mysterious febrile illness afflicting abattoir workers in Brisbane, Australia. He was able to experimentally infect guinea pigs with samples derived from infected patients; however, he could not isolate or identify the causative agent. The elusive nature of this organism led him to name the disease $Q$ fever, or "query" fever (1, 2). Derrick sent infected animal tissues to Burnet and Freeman, who experimentally infected mice and subsequently identified "rickettsial bodies" in their spleens $(3,4)$. Meanwhile, Cox and Davis of Rocky Mountain Laboratories in Hamilton, Montana had discovered a small, filterable agent in ticks that caused disease when injected into guinea pigs (5). A laboratory-acquired infection with the unidentified organism (6) soon led Burnet

and Cox to conclude they were studying the same organism. Thus, the name Coxiella burnetii was chosen to represent the pioneering work of both groups.

\section{Bacteriology}

C. burnetii has a biphasic developmental cycle comprised of large (LCVs) and small cell variants (SCVs) $(7,8)$. LCVs are metabolically active and replicate with a doubling time of $20-45$ hours $(9,10)$. Conversely, SCVs are metabolically inert. Due to their spore-like nature, SCVs are highly resistant to environmental stressors 
including extreme temperatures, desiccation, and changes in osmotic pressure. This hardiness allows $C$. burnetii to persist in the soil and remain infectious for at least 1 year (11). SCVs are the transmissible form and are taken up by alveolar macrophages via phagocytosis (Figure 1-1A). While $C$. burnetii has a tropism for alveolar macrophages $(12,13)$, the bacterium can infect a variety of cell types (1417). Following phagocytosis, the Coxiella-containing vacuole (CCV) subsequently matures along the endocytic pathway, eventually fusing with lysosomes and autophagosomes. While many other intracellular bacteria have evolved mechanisms to block lysosomal fusion (e.g. Mycobacterium tuberculosis, Brucella melitensis) (18), C. burnetii thrives within the highly acidic environment of the phagolysosome. SCVs transition to LCVs in this low $\mathrm{pH}$ environment, establishing a replicative niche known as the parasitophorous vacuole (PV). C. burnetii possesses a type IVB secretion system which spans the bacterial and vacuolar membranes to inject effector molecules into the host cell cytosol (Figure 1-1B). These effector proteins allow the bacterium to hijack host machinery to promote PV biogenesis and bacterial survival (19-24). When triggered by environmental pressure, LCVs can revert to SCVs - thus completing $C$. burnetii's life cycle. C. burnetii also undergoes antigenic phase variation. Phase I organisms possess full-length lipopolysaccharide (LPS) with a unique O-antigen containing two unusual sugars, virenose and dihydrohydoxystreptose (Figure 1-2) $(8,25,26)$. Upon serial passage in eggs, tissue culture, or synthetic media, virulent phase I organisms lose the O-antigen and outer core regions of their LPS and become 
avirulent phase II organisms (27-29). This loss is due to a large chromosomal deletion encompassing multiple LPS biosynthesis genes (30). Phase I organisms can replicate in immunocompetent animals and cause disease, while phase II organisms are rapidly cleared and do not cause disease (31).

\section{Transmission}

C. burnetii colonizes a wide host range, including wild and domestic mammals, marine mammals, reptiles, birds, and arthropods (32). This globally distributed pathogen is primarily spread to humans via inhalation of contaminated aerosols generated from milk, urine, feces, or birth products (Figure 1-3). Livestock are the chief reservoir for human infection, harboring more than $10^{9}$ infectious doses per gram of placental tissue $(33,34)$. As such, this pathogen serves as an occupational hazard for individuals working closely with livestock, including farmers, veterinarians, abattoir workers, and researchers (35-37). A large outbreak resulting from the airborne spread of $C$. burnetii from infected sheep and goat farms occurred in the Netherlands from 2007-2010 $(38,39)$. During this outbreak, more than 4,000 human $Q$ fever cases were reported (40) and it is estimated that at least 40,000 people were infected (41). Parturient dogs and cats are also important sources of infection, causing a familial outbreak in Nova Scotia, Canada (42). Interestingly, the three-toed sloth has emerged as a critical reservoir of infection in the hyperendemic area of Cayenne, French Guiana (43). Livestock and domestic animals in this region were found to be largely seronegative. 


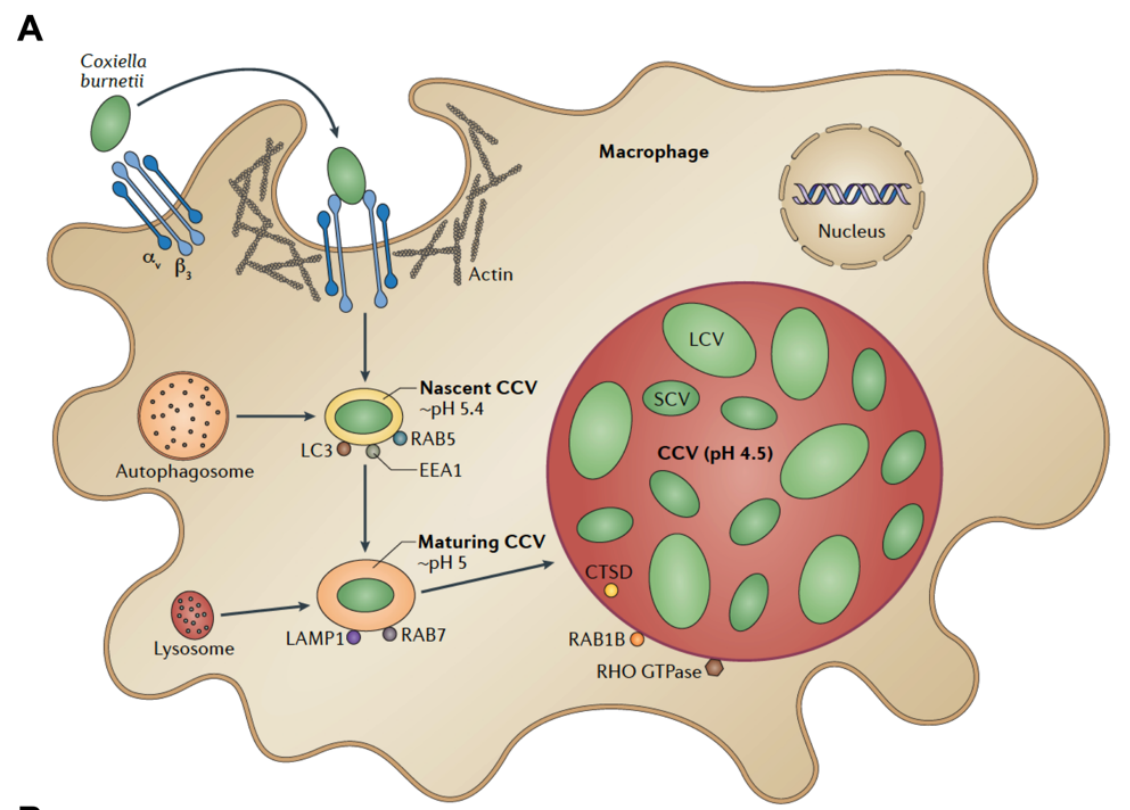

B

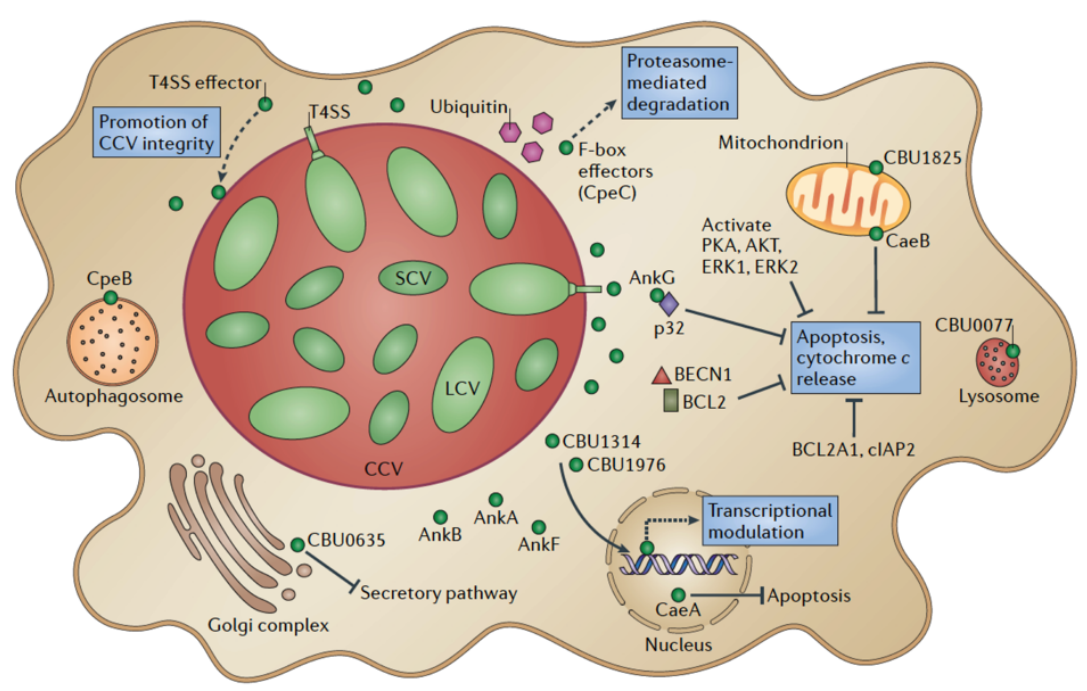

Figure 1-1. The life cycle of $C$. burnetii

(A) C. burnetii binding $a_{v} \beta_{3}$ integrin triggers phagocytosis. Following phagocytosis, the CCV matures along the endocytic pathway, fusing with autophagosomes and lysosomes to establish the low $\mathrm{pH}$ environment of the PV. It is thought that the drop in $\mathrm{pH}$ triggers the transition from SCV to LCV. (B) C. burnetii possesses a type IVB secretion system which injects effector molecules into the host cell. While the majority of these effectors are uncharacterized, they are predicted to modulate host cell signaling pathways to promote $C$. burnetiireplication and PV biogenesis. T4SS, type IV section system.

Adapted from van Schaik EJ et al. 2013. Nature Rev Microbiol 11. 


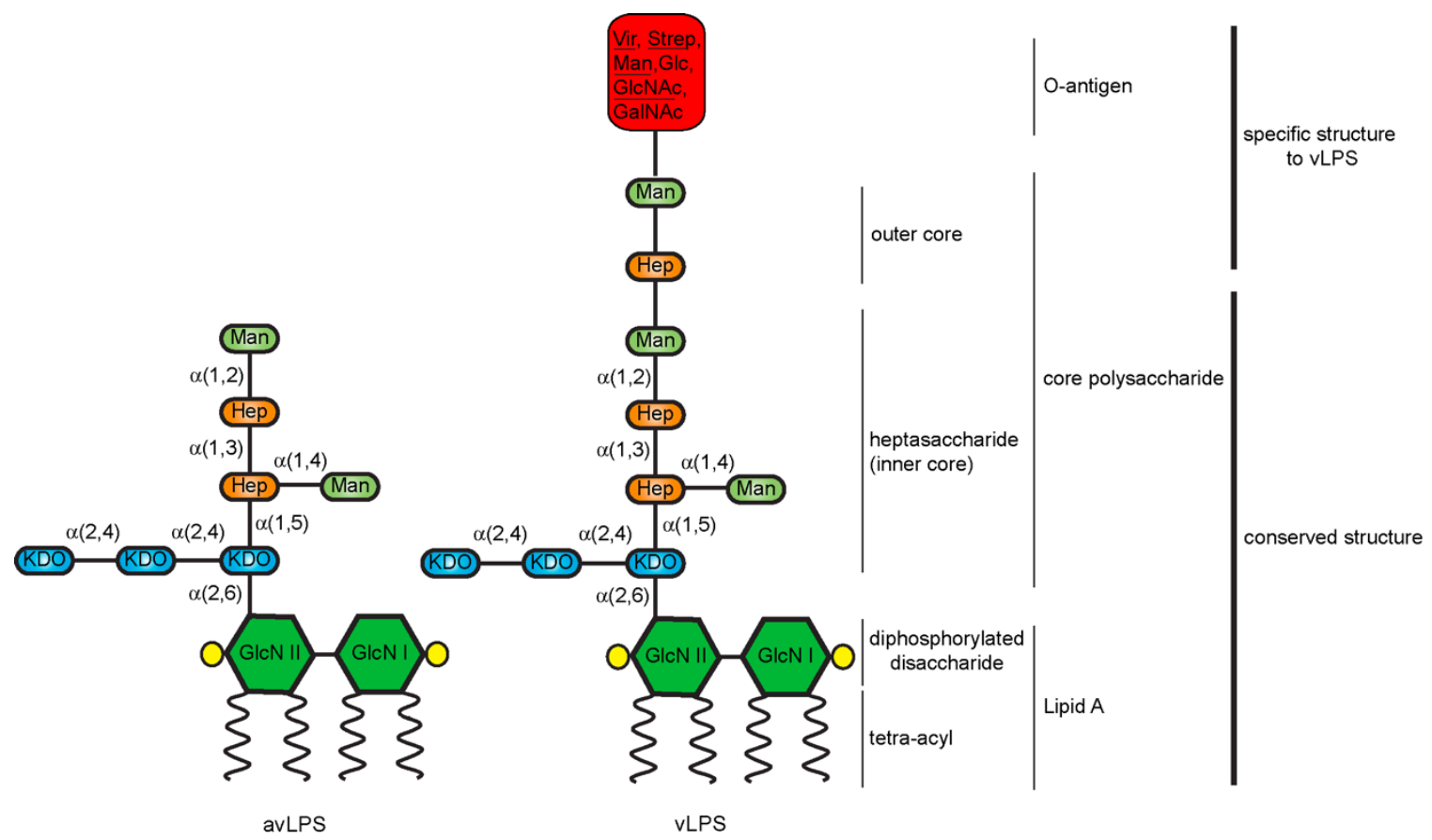

Figure 1-2. C. burnetii LPS phase variation

In nature, $C$. burnetii possesses full-length "smooth" phase I LPS (vLPS) consisting of lipid A, core polysaccharide, and O-antigen. Serial passage of phase I organisms results in the deletion of several LPS biosynthesis genes which produces bacteria with truncated "rough" phase II LPS (avLPS) lacking O-antigen and outer core regions.

Adapted from Barry AO et al. 2012. Cell Host Microbe 12. 
C. burnetii does not cause overt disease in infected animals; however, they may develop reproductive disorders such as infertility and spontaneous abortion. Ingestion of contaminated food or water can also transmit $Q$ fever to humans. In 1974 it was reported that $82 \%$ of the dairy cattle tested in California were seropositive for $C$. burnetii, with $51 \%$ shedding $C$. burnetii in their milk (44). A more recent study (45) found at least $94 \%$ of bulk milk tank samples from dairy herds across the United States to be positive for $C$. burnetii. Still, pasteurization effectively kills $C$. burnetii (46) and has virtually removed the risk of infection from dairy consumption. Similar to its relative Legionella pneumophila (47), C. burnetii is adapted for survival within bodies of water and can remain viable within amoeba for at least 6 weeks $(48,49)$. Furthermore, amoeba containing $C$. burnetii can successfully infect mammalian cell lines, which suggests this is a potentially important reservoir for $C$. burnetii in the environment.

Tick bites are another potential route of exposure, although their role in human transmission is controversial (50). More than 40 species of hard-shell ticks and at least 14 species of soft-shell ticks are known to be positive for $C$. burnetii $(27,41)$. A study in Portugal examined seroconversion rates in humans following various routes of $C$. burnetii infection and found that intradermal (i.d.) inoculation (i.e. the route most closely mimicking a tick bite) achieved $100 \%$ seroconversion $(41,51)$. While this suggests that tick transmission to humans is possible, more than likely they and other arthropod vectors (e.g. fleas, lice, mites, flies) promote the enzootic cycle of infection. 
Although extremely rare, cases of $Q$ fever have been reported through sexual transmission (52) and the transovarial passage from an infected mother to her fetus (53). Moreover, pregnant women can shed large quantities of $C$. burnetii during delivery, which has reportedly caused infections in obstetrical care staff. Blood transfusions are another, albeit uncommon, source of infection. C. burnetii has been demonstrated to survive in stored human blood samples for at least 6 weeks (54) and a screen of blood donors in the Netherlands found that $4.4 \%$ of donors were positive for $C$. burnetii antibodies (55). A subsequent study estimated the risk of infection from a contaminated blood transfusion to be 260 out of every 100,000 donors (56).

In addition to the natural routes of exposure, aerosolization of $C$. burnetii for use as a biological weapon has been previously investigated $(57,58)$. The highly infectious nature of $C$. burnetii $(31,32,59)$, coupled with its prolonged environmental stability (32) and ease of dissemination $(60,61)$, make it a potential bioterror threat. In one epidemiological study (62), it was demonstrated that aerosolized $C$. burnetii could be carried by the wind up to $30 \mathrm{~km}$ from the point of release. With this in mind, it is estimated that the release of $50 \mathrm{~kg}$ of $C$. burnetii 2 $\mathrm{km}$ upwind of a population of 500,000 people would result in approximately 150 deaths and 125,000 incapacitated persons (57). The widespread incapacitating effects of aerosolized $C$. burnetii warrant its designation as a select agent under the Federal Select Agent Program (7 CFR Part 331, 9 CFR Part 121, and 42 CFR Part 73). 


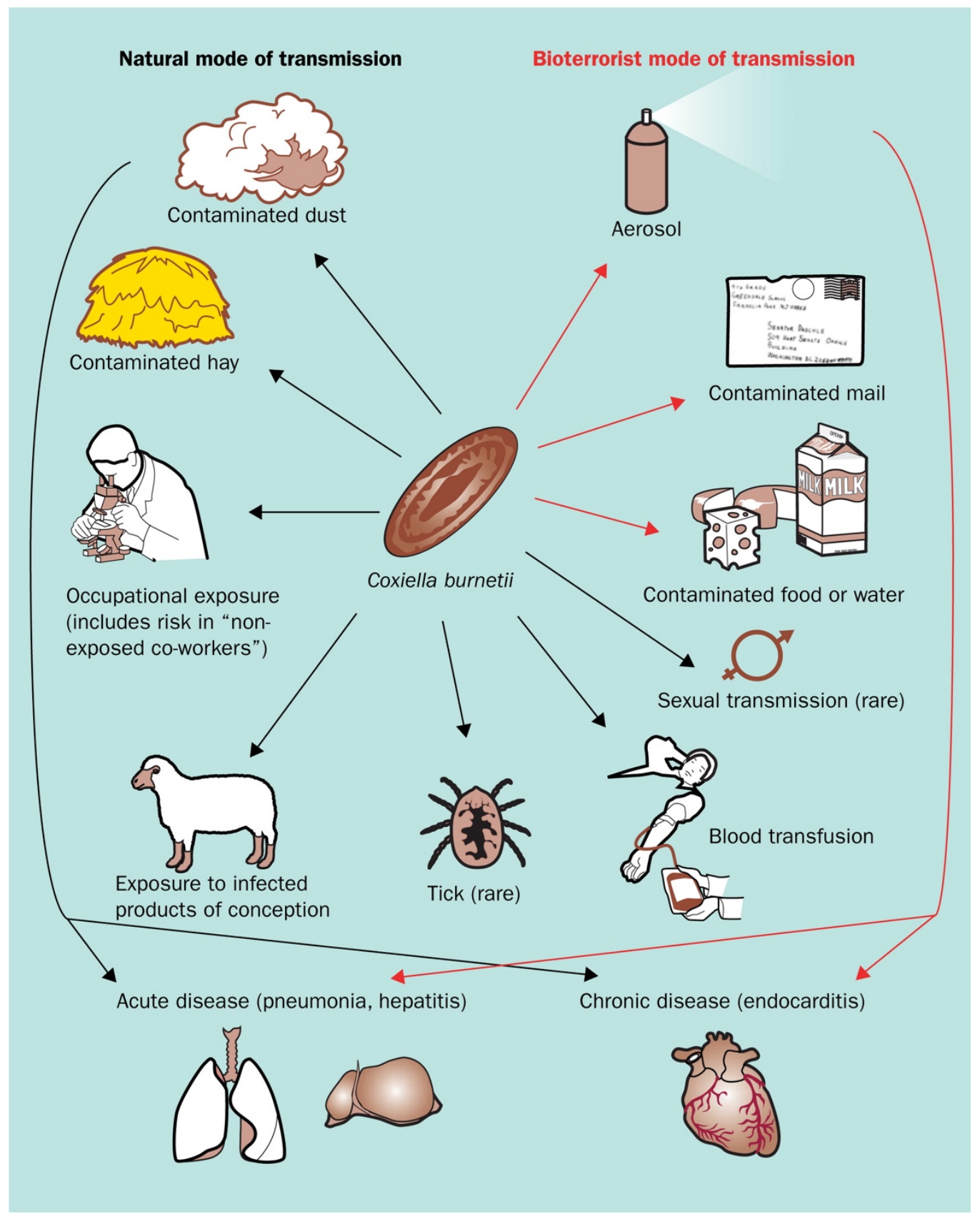

Figure 1-3. Routes of $C$. burnetiitransmission

C. burnetii is transmitted via aerosols from contaminated dust or hay, through aerosols generated from the bodily fluids of infected animals, and by aerosols generated in the laboratory. Intentional release during a bioterrorist attack can also transmit $C$. burnetii. Less common modes of transmission include ingestion of contaminated food or water, tick bite, sexual transmission, and blood transfusion.

Adapted from Madariaga MG et al. 2003. Lancet Infect Dis 3. 


\section{Signs \& symptoms}

Acute $Q$ fever develops as a flu-like illness with fever, chills, fatigue, headaches, and body aches approximately 2-3 weeks following exposure to $C$. burnetii. Gastrointestinal (e.g. nausea, vomiting, diarrhea, stomach pain) and respiratory (e.g. non-productive cough, pneumonia) signs are also common. However, 50\% of those infected will remain asymptomatic (63). Less than $5 \%$ of acutely infected patients will develop severe, persistent infections months to years after initial exposure. This form of the disease, historically called chronic $Q$ fever, involves diverse infection foci (e.g. endocarditis, hepatitis, lymphadenitis, osteomyelitis). The most common presentation of chronic infection is endocarditis $(27,48,64,65)$, representing $68 \%$ of the persistent focalized infections reported to the French National Referral Center for Q Fever in Marseille, France between 2007-2015 (66). Of those who develop chronic disease, fatality is observed in $25-60 \%$ of patients when left untreated (63).

Certain "at-risk" groups are predisposed to persistent infections including, immunocompromised persons, pregnant women, and those with pre-existing heart valve damage (67). Similar to infected animals, expectant mothers who become infected may develop reproductive disorders involving miscarriage, pre-term delivery, or low birth weight $(53,68)$. Another complication of acute disease, postQ fever fatigue syndrome (QFS), is characterized by severe fatigue years after the initial infection. QFS patients also apparently suffer from decreased quality of life compared to healthy controls (69-74). Limonard and colleagues assessed the 
health status of patients 4 years after the Dutch outbreak and found that $46 \%$ of patients experienced "undue fatigue," while $50 \%$ of patients suffered a "severely impaired general quality of life" (74). This was a follow-up assessment of a casecontrol study performed 1 year following the outbreak (70), and no significant changes in sub-domain scores were found. This indicates QFS persistence, although whether QFS is associated with persistent infection remains controversial. In the aforementioned study, patients with "possible, probable or proven chronic $Q$ fever" were excluded, which indicates that persistent infection is not a requirement for QFS. However, non-viable Coxiella antigens have been found in patients up to 12 years after acute infection, which may cause chronic immune stimulation and contribute to QFS $(75,76)$.

\section{Diagnosis \& testing}

The non-descript symptoms associated with $Q$ fever make diagnosis challenging. Patients are frequently misdiagnosed and fail to receive appropriate laboratory testing. Serology to detect $\lg M$ and $\lg G$ antibodies against $C$. burnetii phase variants is the standard diagnostic (77). Antibody titers less than 1:16 are considered negative. Elevated IgM or higher phase II titers is suggestive of acute $Q$ fever. Conversely, elevated IgG or higher phase I titers are indicative of persistent infection. If a persistent infection is suspected, additional testing may be performed, including chest x-rays or an echocardiogram. Polymerase chain reaction (PCR) to detect $C$. burnetii DNA can also aid in diagnosis $(78-80)$, however, it is not as informative in terms of differentiating active from past infection. 
A defined acidified citrate cysteine medium (ACCM-D) has been developed which mimics the intracellular replicative niche of $C$. burnetii and allows axenic growth of the bacteria. This new technology may be useful for diagnostics as it will allow the enumeration of live $C$. burnetii.

\section{Treatment}

Acute $Q$ fever is often self-limiting and may not require antibiotic treatment. However, considering the threat of chronic manifestations, early antibiotic intervention is recommended. Doxycycline for $2-3$ weeks is the first line of treatment for children and adults with acute disease (63). Consequently, many doxycycline resistant strains have emerged (81-83). Alternative antibiotic therapies for acute $Q$ fever include fluoroquinolones, rifampicin, and telithromycin (84); however, resistant strains have also been reported for these compounds $(85,86)$. Telithromycin is active against at least 13 C. burnetii isolates, including 3 doxycycline resistant strains (82), however, at least 1 strain in hyperendemic Cayenne, French Guiana is telithromycin-resistant. To date, strains isolated from this region remain doxycycline sensitive (87).

In the case of persistent infection, long-term ( $\geq 18$ months) administration of doxycycline and hydroxychloroquine is the preferred treatment $(48,88,89)$. The intracellular lifestyle of $C$. burnetii has complicated treatment efforts, as the acidic $\mathrm{pH}$ of the phagolysosome inhibits antibiotic activity (90). Raoult et al. demonstrated that doxycycline sensitivity could be restored when used in conjunction with hydroxychloroquine, an alkalinizing agent which reduces the $\mathrm{pH}$ of the $\mathrm{PV}$. 
However, as mentioned previously, even with the recommended antibiotic regimen, one in three $Q$ fever patients continue to experience diminished health two years post-diagnosis (72).

\section{Prevention}

There is currently no FDA-approved vaccine available against $C$. burnetii infection due to a high incidence of adverse reactions in vaccinated populations. It remains unclear what vaccine components elicit these delayed-type hypersensitivity (DTH) reactions. This, coupled with a lack of knowledge regarding the immunological mechanisms of vaccine protection, have prevented the rational design of a safe and effective new-generation vaccine against $Q$ fever. Multiple animal models of experimental $Q$ fever have been developed as surrogates to study vaccine immunity. This section will highlight those animal models as well as describe the $Q$ fever vaccine research pipeline to date. The mechanisms of vaccine immunity elucidated thus far will also be discussed.

\section{Animal models}

\section{Guinea pigs}

Guinea pigs represent perhaps the best model for $Q$ fever research $(1,3,91-99)$. They are sensitive to low doses of $C$. burnetii (1), with disease progressing similarly to human $Q$ fever. Following subcutaneous (s.c.) or intraperitoneal (i.p.) inoculation, a short-lived fever develops with some animals exhibiting further signs of illness, including lethargy, anorexia, weight loss, and ruffled fur (1). I.p. challenge with purified LPS generates a similar response (96). Furthermore, 
guinea pigs develop pneumonia following aerosol challenge, with peak severity coinciding with the peak of the fever response (98). Some animals remain afebrile throughout infection, not unlike humans. Regardless of the severity of clinical signs, postmortem examination of infected guinea pigs reveals marked spleen and liver enlargement $(1,96)$. Splenomegaly, along with fever development and specific antibody production, has been used to assess vaccine efficacy.

The Samuel and Heinzen labs have utilized the guinea pig aerosol (100) and i.p. (101) challenge models, respectively, and demonstrated that the clinical course of disease is strain-dependent. C. burnetii strains differ in LPS profile $(28,102)$, plasmid type (103) and chromosomal DNA restriction endonuclease fragment patterns (104). These differences are associated with a predilection towards acute versus chronic disease and are the basis for the genomic groups defined by Hendrix and colleagues (104).

\section{Non-human primates}

Non-human primates also develop disease similar to humans (3), although they are not commonly used due to the high costs associated with their housing and care. After i.d. and/or s.c. inoculation, rhesus monkeys develop a fever and other outward signs of illness including lethargy and loss of appetite (3). Non-human primates also develop pneumonia when challenged with aerosolized C. burnetii and show splenomegaly and hepatomegaly upon necropsy $(105,106)$. Rhesus monkeys may be more susceptible to $C$. burnetii than cynomolgus monkeys, as they have higher bacteremia and a longer fever duration (106). However, 
cynomolgus monkeys seem to develop more severe pneumonia. As such, the cynomolgus monkey aerosol challenge model has been used to study vaccine efficacy $(107,108)$. A reduction in the frequency or duration of clinical signs (e.g. pneumonia, fever) and an increase in specific antibody titers are considered correlates of protection in this model.

\section{Mice}

Unlike guinea pigs and non-human primates, immunocompetent mice generally show no overt signs of disease. However, disease susceptibility is straindependent $(109,110)$. A/J mice are the most susceptible strain and one of the only lethal murine models of Q fever. Scott et al. (109) reported 100\% mortality following i.p. inoculation of $\mathrm{A} / \mathrm{J}$ mice with $10^{7} \mathrm{C}$. burnetii Nine Mile phase I (NMI). Conversely, resistant C57BL/6 mice had 100\% survival at this challenge dose. Other susceptible strains include $\mathrm{BALB} / \mathrm{c}$ and $\mathrm{C} 3 \mathrm{H} / \mathrm{He}$ mice. Regardless of the degree of outward disease, mice develop enlarged livers and spleens in response to $C$. burnetii infection (3). Intranasal (i.n.), intratracheal (i.t.), and aerosol models of $Q$ fever have also been developed in mice and cause pneumonia (111-113).

Over the last few decades, mice have been used to study the host response to vaccination $(99,114-121)$. Williams and colleagues have used C57BL/10ScN endotoxin non-responder mice to study vaccine efficacy $(114,115,122)$, while others have used $\mathrm{A} / \mathrm{J}(99,116)$ and BALB/C $(117,123-126)$ strains. Although C57BL/6 mice are resistant to $C$. burnetii infection, they are continually used in vaccine immunity studies due to the availability of immunodeficient strains on the 
C57BL/6 background $(119,127,128)$. The degree of splenomegaly has been shown to correlate with the splenic bacterial burden (129), making it a good readout of vaccine protection. As with guinea pig and non-human primate models, specific antibody production is also evaluated to assess protection.

\section{Human vaccines}

\section{Whole-cell vaccines}

Formalin-inactivated whole-cell vaccines have been under research for decades. In the early 1940s, Bengtson made vaccine preparations of killed C. burnetii from homogenates of infected mouse and guinea pig spleens as well as infected yolk sacs and mouse livers (91). She found almost complete protection against a subsequent challenge from these formulations. Guinea pigs who received varying doses $(2.5-20 \%)$ of vaccine had a lower incidence of fever compared to unvaccinated controls, which spiked fevers soon after challenge.

Smadel et al. made similar preparations using Henzerling and Dyer strains of $C$. burnetii (92). Vaccinated guinea pigs had a shorter duration of fever following challenge, with Henzerling vaccinates developing a stronger protective response as measured by fever prevalence and duration. This study was also the first to evaluate human reactions to vaccination. Over $90 \%$ of human vaccinates developed specific complement-fixing antibodies, with little to no adverse reactions. This is indicative of a protective response; however, no human challenge experiments were performed here.

A later study in California (130) followed the development of antibody responses in laboratory personnel vaccinated with one or multiple injections of formalin- 
inactivated Henzerling strain. Only 12 of 23 vaccinates developed sufficient antibody titers following the first injection, leading investigators to conclude that at least two injections are necessary to generate an appropriate antibody response in the majority of vaccinates. However, it was also observed that severe local reactions occurred during booster injections which presents a complication for this strategy.

Similar studies in the United Kingdom demonstrated that laboratory personnel receiving $C$. burnetii vaccine were protected from reinfection for 5 years (131). Only one vaccinated person contracted $Q$ fever, and it was determined that exposure to C. burnetii occurred before sufficient time had passed for vaccine-induced protection. All other cases of $Q$ fever in the department occurred in unvaccinated persons. Again, it was observed that following booster injections, abscesses sometimes formed at the site of inoculation. These occasionally progressed in severity as to require surgical intervention.

Following continuous reports of vaccine reactions, Lackman and colleagues developed an intradermal sensitivity test (132). They demonstrated that pre-testing for immunity can prevent adverse reactions, which were likely caused by previous sensitization. This hypothesis was later proven by Luoto et al. (133) who demonstrated that reactions were prevented by only vaccinating serologically negative persons. The development of sensitivity testing was a great stride in the field that is still implemented today. However, pre-testing for immunity is timeconsuming and costly. Considering $C$. burnetii has the potential for use as a 
biological weapon, it is of utmost importance to develop a non-reactogenic vaccine that can be used for mass vaccination.

Around this time, it was also discovered that $C$. burnetii undergoes phase variation, as previously described. Ormsbee et al. established that phase influences vaccine potency. Specifically, phase I vaccines possess 100-300 times greater protective potency than phase II vaccines - regardless of the strain used (95). This was reflected in the levels of complement-fixing and agglutinating antibodies. While this discovery contributed to the field, formalin-inactivated phase I vaccines are still capable of inducing tissue reactions. A study from Rocky Mountain Laboratories found that over one-third of vaccinated personnel had some vaccine reaction (134). Of those that developed a tissue reaction, approximately $30 \%$ resulted in abscess formation with 4 vaccinates requiring surgical intervention. This study highlights the importance of sensitivity screening, although a non-reactogenic vaccine is still desired.

A formalin-inactivated whole-cell vaccine produced from the Henzerling strain in phase I (Q-VAX ${ }^{\circledR}$ ) has been shown to elicit long-lasting protection in animal models and human vaccinees $(35,117,135,136)$. This vaccine was licensed for use in Australia following an extensive 5-year survey in abattoir workers $(35,136)$. Workers were pre-screened before naïve volunteers were vaccinated and subsequently monitored for the development of $Q$ fever. The initial study found near-complete protection for up to two years following vaccination (35). A retrospective survey of vaccinates found this protection could be maintained for at 
least 5 years. Only two vaccinated people developed $Q$ fever compared to over 50 of the unvaccinated personnel (136). Despite its high protective efficacy, Q-VAX ${ }^{\circledR}$ is not approved for use in the United States due to adverse reactions, especially in previously sensitized individuals $(35,92,130,131,134,135)$.

\section{Chloroform:methanol residue vaccines}

A promising alternative to formalin-inactivated vaccines was developed through chloroform:methanol extraction. Williams and Cantrell demonstrated that vaccination with chloroform:methanol residue (CMR) reduced or completely negated pathological changes in $\mathrm{C} 57 \mathrm{BL} / 10 \mathrm{ScN}$ endotoxin non-responder mice (114). In contrast, mice that received whole-cell suspensions displayed gross pathological changes, including hepatomegaly and splenomegaly. When compared to the whole-cell vaccine, CMR displayed increased protection ( $80 \%$ vs. $50 \%$ ) in a lethal challenge of $\mathrm{C} 57 \mathrm{BL} / 10 \mathrm{ScN}$ endotoxin non-responder mice (115). CMR was also demonstrated to be protective in $\mathrm{A} / \mathrm{J}$ mice and guinea pigs following aerosol challenge with negligible adverse effects (99). It appeared that this was a promising non-reactogenic vaccine candidate to replace Q-VAX ${ }^{\circledR}$. Upon testing in cynomolgus monkeys, Waag et al. demonstrated that CMR provided equivalent protection when compared to $\mathrm{Q}-\mathrm{VAX}{ }^{\circledR}$ (108). This, coupled with decreased tissue reactions, further suggested CMR as a good alternative to whole-cell vaccines like Q-VAX ${ }^{\circledR}$. However, upon further testing, it was shown that CMR could induce pathological changes similar to $\mathrm{Q}-\mathrm{VAX}{ }^{\circledR}$ when administered at higher doses (137). This finding has prevented the approval of the CMR vaccine for use against $C$. 
burnetii. A recent study (126) demonstrated that CMR administered i.t. could enhance mucosal immunity in the lungs of BALB/c mice compared to the s.c. vaccination route in an aerosol infection model. However, reactogenicity issues have still not been addressed.

\section{Other vaccine strategies}

Several other vaccine strategies have been tested or are currently in development. A live attenuated vaccine was developed from the $M-44$ strain of $C$. burnetii to vaccinate Soviet volunteers (137-139). However, it was demonstrated that organisms persisted for a long time in inoculated animals, which is indicative of the potential for reactivation (140-142).

Various cellular fractions have also been explored. Anacker and colleagues vaccinated guinea pigs with purified cell wall, protoplasm, and trichloroacetic acid (TCA) extracts (94). They found purified cell wall to have similar immune properties as whole-cell suspensions. Conversely, vaccination with protoplasm did not elicit a protective response. This suggests that the protective epitopes of $C$. burnetii are found in the cell wall. TCA extracts did elicit an immune response, although it was markedly less than whole-cell and cell wall vaccines. Protein and carbohydrate fractions have also been purified for vaccination (97). Both fractions were found to contribute to the protection of vaccinated animals as measured by cell migration assays.

Recombinant protein vaccines have been investigated using reportedly immunodominant C. burnetii proteins. In the early 1990s, a 62 kilodalton (Kd) 
protein annotated as a "common antigen" of $C$. burnetii as well as two surface antigens (P1 and P2) were purified and tested as potential vaccine candidates in A/J mice (116). The $62 \mathrm{Kd}$ and P2 proteins were unable to elicit an appropriate immune response, however, P1 did appear to elicit strong anti-phase I antibody production. Furthermore, vaccination with $\mathrm{P} 1$ resulted in a greater reduction in spleen weight and splenic bacterial burden than vaccination with purified phase I LPS (116). This data highlighted recombinant $\mathrm{P} 1$ as a promising vaccine candidate. P1 was visited again a decade later along with Com1, Cb-Mip, and P28 - all of which reacted strongly with immune serum (143-145). None of these proteins were able to elicit a protective immune response on their own, as indicated by splenomegaly equivalent to unvaccinated controls $(137,146)$. A purified $67 \mathrm{Kd}$ outer membrane protein was tested for immunogenicity and protectivity in mice and guinea pigs by Zhang et. al in 1994 (147). This protein induced specific antibody production, in vitro lymphocyte proliferation, and protected $100 \%$ of vaccinated mice and guinea pigs following challenge with $10^{3} \quad \mathrm{ID}_{50}$ virulent $C$. burnetii. These data indicated that the $67 \mathrm{Kd}$ outer membrane protein was a promising candidate for a protein subunit vaccine, however additional data has not been published at this time.

An LPS vaccine has also been developed and tested in $\mathrm{A} / \mathrm{J}$ mice. Upon lethal aerosol challenge, phase I LPS was able to significantly reduce the number of deaths as well as the bacterial burden in the spleen (116). Recently, phage display technology was used to develop a peptide mimic vaccine against $Q$ fever which 
expresses the protective epitopes of phase I LPS (118). Initial work suggests that an identified peptide (m1E41920) elicits strong anti-C. burnetii antibody responses. Immune serum from vaccinated mice is also protective when passively transferred to naïve animals (118). Preclinical safety and efficacy studies in additional animal models will be required before seeking regulatory approval per the FDA Animal Rule $(148,149)$.

Since 2017, a team of scientists with expertise spanning epitope generation and optimization, assay development, RNA replicon generation, and vaccine safety and efficacy testing formed a consortium called Q-vaxcelerate to engineer a nonreactogenic $T$ cell-targeted $Q$ fever vaccine (150). To date, the group has published work describing epitope leads selected with an immunoinformatic approach for predicted recognition by major histocompatibility complex class I (MHC-I) and class II (MHC-II) molecules $(151,152)$. While these epitopes were immunostimulatory ex vivo, it remains to be determined if a vaccine encompassing these epitopes will be protective considering the failure of previous subunit vaccine attempts.

\section{Animal vaccines}

Due to the high incidence of spontaneous abortions in infected ruminants, there is a significant economic impact associated with $C$. burnetii infection. Therefore, the prevention and/or reduction of livestock infections through vaccination is critical. Not only would vaccination reduce economic losses, but it would also potentially eliminate the chief source of human transmission. A formalin-inactivated Nine Mile 
phase I whole-cell vaccine is commercially available for ruminant animals in Europe $\left(\right.$ Coxevac $\left.^{\circledR}\right)$. This vaccine was initially authorized by the European Medicines Agency (EMA) under "exceptional circumstances" during the Dutch outbreak $(153,154)$ and has since achieved full regulatory approval. Vaccination of naïve dairy goats and sheep, coupled with the culling of infected herds, resulted in an approximately 5-fold decrease in the number of human infections from 20092010 (40). Coxevac ${ }^{\circledR}$ is currently indicated for cattle and goats, however, off-label use for sheep is common. Several studies in dairy cows $(155)$ and goats $(156,157)$ indicate that Coxevac ${ }^{\circledR}$ protects naïve animals. Additionally, it has been shown that vaccination can reduce bacterial shedding in milk, uterine fluids, and feces from infected animals (156-162). Furthermore, Coxevac ${ }^{\circledR}$ reduces the number of abortions in goats $(156,157)$. However, multiple reports have demonstrated that C. burnetii continues to persist in environmental samples $(158,159)$, which suggests the potential reinfection of vaccinated populations when immunity wanes. The EMA states that the duration of vaccine immunity is 280 days from completion of the vaccination course in cattle and 1 year in goats (154).

\section{Ongoing challenges}

Presently, there are no $Q$ fever vaccines approved for use in the United States. Formalin-inactivated and CMR vaccines are protective but remain associated with adverse reactions in sensitized individuals. Live attenuated vaccines, cellular fractions, and protein subunit vaccines have proved unsuccessful in eliciting a protective response. There is promise in the development of a peptide mimic 
vaccine, although further work is needed. Until recently, vaccines have been developed empirically, without knowledge of the immunological mechanisms by which they protect (163). However, recent pandemics like HIV have led scientists to begin looking at the mechanisms behind vaccine protection to develop more targeted therapeutics.

\section{Immunological mechanisms of vaccination}

It has previously been demonstrated that both humoral and cell-mediated immunity (CMI) contribute to host defense against $C$. burnetii $(98,117,119,164-175)$. The production of complement-fixing and agglutinating antibodies has been measured as a correlate of immunity in man and experimental animals since the bacterium was first discovered $(98,164-167,169)$. B cells are required for formalininactivated whole-cell C. burnetii Nine Mile phase I vaccine (PIV)-mediated protection, as B cell-deficient $\mu \mathrm{MT}$ mice have significantly worse disease than wildtype (WT) mice (119). These B cells produce protective IgM and IgG, which are sufficient for generating a protective response following passive transfer to immunocompetent mice. Furthermore, passive transfer of IgM from CD4-deficient (CD4 KO) mice is protective, which suggests $\mathrm{CD}^{+} \mathrm{T}$ cell-independent mechanisms contribute to vaccine immunity. In contrast, passive transfer of IgG from CD4 $\mathrm{KO}$ mice is not protective. This indicates that $\mathrm{CD} 4^{+} \mathrm{T}$ cells are required for a protective IgG response. T cell-deficient athymic mice exhibit impaired immunity compared to WT mice upon passive immunization (168), which further suggests that $\mathrm{T}$ cells are required for antibody-mediated immunity. Indeed, Zhang 
et. al (117) found IgG2a to be the predominant $\lg G$ isotype generated in response to vaccination with PIV and B cell production of this isotype is largely driven by $T$ cell secretion of interferon-gamma (IFN- $\gamma$ ) (176). Additionally, only adoptive transfer of immune T cells, not immune B cells, from PIV-vaccinated BALB/c mice to naïve severe combined immunodeficient (SCID) mice reduces disease severity following i.p. challenge (117). This indicates that while specific antibody production and B cells are necessary for vaccine protection, they are not sufficient. Conversely, T cells are necessary and sufficient for vaccine-mediated immunity. More specifically, either $\mathrm{CD} 4^{+}$or $\mathrm{CD} 8^{+} \mathrm{T}$ cells are sufficient to generate protection following vaccination with PIV (119). 


\section{CHAPTER 2: MATERIALS AND METHODS}

\section{Animals}

Four to 12-week-old male and female specific pathogen-free (SPF) mice were used in these studies (Table 2-1). Animals were housed in sterile microisolator cages in a conventional animal facility or in an animal biosafety level 3 (ABSL-3) facility at the MU-LIDR. Animals were provided food and water ad libitum. All research involving animals was conducted in accordance with the Animal Care and Use guidelines, and all animal use protocols were approved by the Animal Care and Use Committee at the University of Missouri. All infections were conducted in an ABSL-3 facility at the MU-LIDR.

\section{Bacterial strains}

C. burnetii NMI clone 7 (RSA 493) and Nine Mile phase II (NMII) clone 4 (RSA 439) were propagated in acidified citrate cysteine medium-2 (ACCM-2) as previously described (177). Bacteria were purified by centrifugation at 15,000 x g for $30 \mathrm{~min}$, followed by 2 washes with sterile $1 \mathrm{X}$ phosphate-buffered saline (PBS). NMI was handled under biosafety level 3 (BSL-3) conditions at the MU-LIDR.

\section{Vaccination}

Purified C. burnetii NMI and NMII were inactivated for $48 \mathrm{~h}$ in $10 \%$ formalin followed by dialysis in deionized water. Antigen concentrations were then measured using a Micro BCA protein assay kit (Pierce, Rockford, IL) per manufacturer instructions. 


\begin{tabular}{|c|c|c|c|c|c|c|c|c|c|}
\hline & & 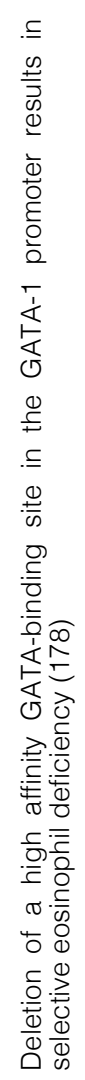 & 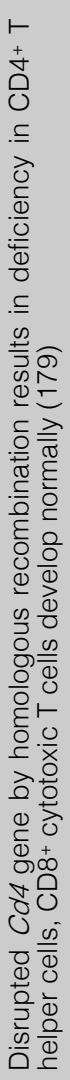 & 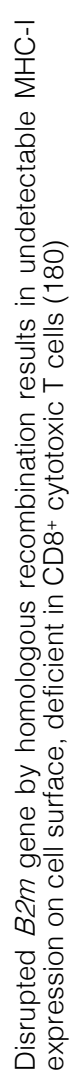 & 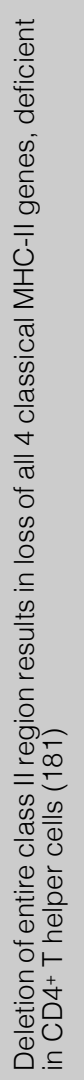 & 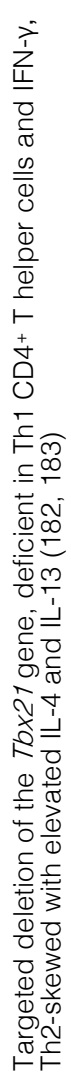 & 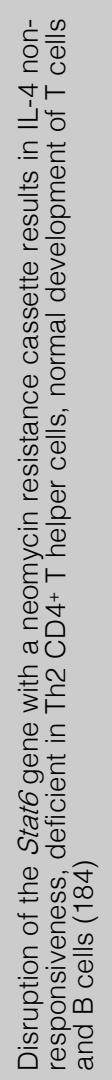 & 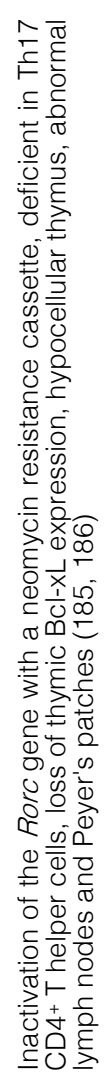 & 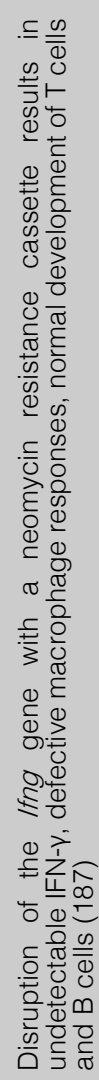 \\
\hline 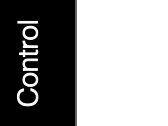 & & $\frac{\frac{0}{\infty}}{\frac{\vec{\alpha}}{\grave{\Phi}}}$ & 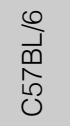 & $\begin{array}{l}\stackrel{0}{\vec{p}} \\
\stackrel{0}{\hat{0}} \\
\stackrel{0}{0}\end{array}$ & $\begin{array}{l}\stackrel{0}{\vec{p}} \\
\hat{\infty} \\
\hat{\jmath} \\
0\end{array}$ & $\begin{array}{l}\stackrel{0}{\partial} \\
\stackrel{0}{0} \\
\stackrel{0}{0} \\
0\end{array}$ & $\begin{array}{l}\stackrel{0}{\vec{p}} \\
\stackrel{0}{0} \\
\tilde{\delta}\end{array}$ & $\begin{array}{l}\stackrel{0}{\partial} \\
\stackrel{0}{0} \\
\hat{\jmath} \\
0\end{array}$ & $\begin{array}{l}\stackrel{0}{\partial} \\
\stackrel{0}{0} \\
\tilde{b}\end{array}$ \\
\hline $\begin{array}{l}\overline{8} \\
\varnothing \\
8\end{array}$ & 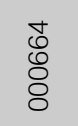 & 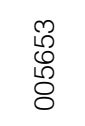 & 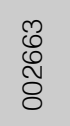 & $\begin{array}{l}\hat{\infty} \\
\overbrace{\hat{N}} \\
\stackrel{\delta}{0}\end{array}$ & 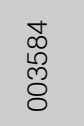 & \begin{tabular}{l}
$\infty$ \\
\multirow{0}{0}{} \\
0 \\
0 \\
0
\end{tabular} & 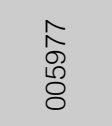 & $\begin{array}{l}\overline{\hat{N}} \\
\hat{5} \\
8\end{array}$ & $\begin{array}{l}\text { o } \\
\underset{N}{ } \\
\varnothing\end{array}$ \\
\hline 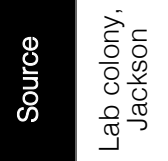 & 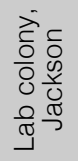 & 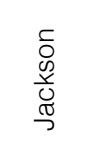 & 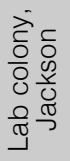 & 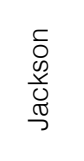 & 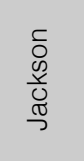 & 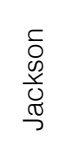 & 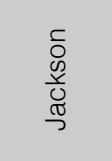 & 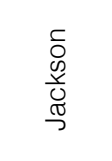 & 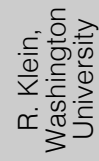 \\
\hline $\begin{array}{l}\text { 을 } \\
\frac{\overline{7}}{2} \\
\frac{0}{0} \\
\frac{0}{0} \\
\frac{0}{4}\end{array}$ & & $\begin{array}{l}\frac{\mathbb{6}}{8} \\
\frac{0}{0} \\
\frac{0}{0} \\
8\end{array}$ & $\begin{array}{l}\stackrel{0}{x} \\
\stackrel{1}{\Delta} \\
\text { Oे }\end{array}$ & 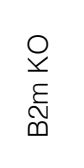 & $\begin{array}{l}\stackrel{0}{\underline{x}} \\
\overline{\bar{j}} \\
\frac{1}{\Sigma} \\
\frac{1}{\Sigma}\end{array}$ & 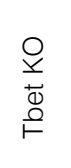 & $\begin{array}{l}\frac{\partial}{y} \\
0 \\
\frac{0}{\pi} \\
\dot{\omega n}\end{array}$ & 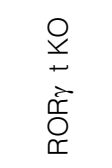 & $\begin{array}{l}\stackrel{ }{\underline{y}} \\
\grave{i} \\
\underline{\underline{u}}\end{array}$ \\
\hline$\frac{\vec{Q}}{\vec{\sigma}}$ & $\begin{array}{l}\stackrel{0}{\vec{p}} \\
\stackrel{0}{b} \\
\tilde{b}\end{array}$ & 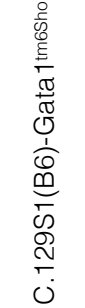 & 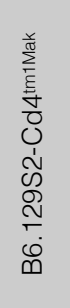 & 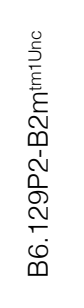 & 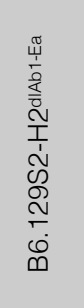 & 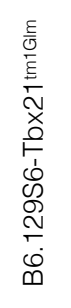 & 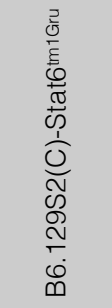 & 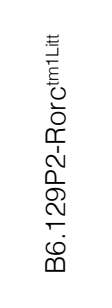 & 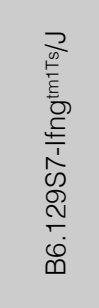 \\
\hline
\end{tabular}


Mice were vaccinated s.c. with $10 \mu \mathrm{g}$ PIV or formalin-inactivated C. burnetii NMII whole-cell vaccine (PIIV) in $50 \mu$ sterile 1 X PBS with $50 \mu$ l aluminum hydroxide adjuvant (Alhydroge ${ }^{\circledR}$ adjuvant 2\%; InvivoGen, San Diego, CA) for a final delivered volume of $100 \mu \mathrm{l}$. Unvaccinated control mice were given $50 \mu \mathrm{l}$ PBS with $50 \mu \mathrm{l}$ adjuvant for a final delivered volume of $100 \mu \mathrm{l}$. Vaccines were prepared with a 1:1 ratio of antigen/adjuvant per manufacturer instructions. Vaccinated and adjuvant control mice were then either challenged to assess protection or sacrificed to assess cellular responses via fluorescence-activated cell sorting (FACS) or enzyme-linked immunosorbent assay (ELISA).

\section{Infection}

Vaccinated and adjuvant control mice were challenged 7,14 , or 28 days postvaccination (dpv) by i.p. injection with either $1 \times 10^{7}$ or $5 \times 10^{7}$ C. burnetii NMI in 400 $\mu \mathrm{l}$ sterile 1X PBS. Mice were weighed throughout infection and relative body weight (current weight/day 0 weight) was calculated to monitor disease progression. The protective efficacies of PIV and PIIV were evaluated 14 days post-infection (dpi) by comparing percent splenomegaly [(spleen weight/body weight)x100] against unvaccinated controls. Bacterial burden in the spleen was also measured using real-time quantitative PCR (qPCR).

\section{qPCR}

Spleen pieces were weighed, diluted 20X in lysis buffer (1M Tris, 0.5M EDTA, 7 $\mathrm{mg} / \mathrm{ml}$ glucose, $28 \mathrm{mg} / \mathrm{ml}$ lysozyme), homogenized and filtered through a $100 \mu \mathrm{m}$ 
nylon mesh to remove any connective tissue. $10 \mu$ Proteinase $\mathrm{K}(20 \mathrm{mg} / \mathrm{ml})$ was then added to $200 \mu \mathrm{l}$ of filtered spleen homogenate and incubated at $60^{\circ} \mathrm{C}$ overnight. Next, $21 \mu \mathrm{l} 10 \%$ SDS was added to samples and incubated at room temp for $1 \mathrm{~h}$. Finally, DNA was extracted using the High Pure PCR Template Preparation kit (Roche, Indianapolis, IN) as directed by the manufacturer. Bacterial burden was determined by quantifying com1 gene copy numbers using a standard curve with SYBR Green (Applied Biosystems, Foster City, CA) on an Applied Biosystems StepOnePlus real-time PCR system. The standard curve was generated using recombinant plasmid DNA (com1 gene ligated into pBluescript vector). Splenic bacterial burden was calculated using the following equation, $[((($ genomic equivalents $\mathrm{x}$ lysis buffer $) / 2) \times$ spleen weight $) /$ spleen piece weight $]$.

\section{Spleen cell isolation}

Spleen cells were harvested from BALB/c, C57BL/6, and CD4 KO mice 7 or 14 dpv or adjuvant treatment. Briefly, spleens were removed and homogenized. The cell suspension was then filtered through a $100 \mu \mathrm{m}$ nylon mesh to remove any connective tissue. Spleen cells were pelleted by centrifugation at $500 \mathrm{xg}$ for $8 \mathrm{~min}$ and resuspended in $5 \mathrm{ml} \mathrm{ACK} \mathrm{lysis} \mathrm{buffer} \mathrm{for} 5 \mathrm{~min}$ at room temp to lyse red blood cells. Remaining cells were then pelleted by centrifugation at $500 \mathrm{xg}$ for $8 \mathrm{~min}$ and resuspended in $2 \mathrm{ml}$ FACS buffer (PBS supplemented with $0.5 \%$ BSA $+2 \mathrm{mM}$ EDTA $+0.1 \%$ sodium azide) for counting 


\section{Flow cytometry}

Approximately $1 \times 10^{6}$ cells/well were added to a 96 -well plate for flow cytometry. Briefly, cells were blocked for 15 min at $4^{\circ} \mathrm{C}$ with Fc Block (BD Biosciences) in FACS buffer. Cells were then stained for $30 \mathrm{~min}$ at $4^{\circ} \mathrm{C}$ with an antibody cocktail containing CD45, CD11b, CD125, Siglec-F, CD19, and CD45R in FACS buffer (Table 2-2). Cells were pelleted by centrifugation at $500 \times \mathrm{g}$ for $8 \mathrm{~min}$, followed by 2 washes with FACS buffer. A 2\% paraformaldehyde solution was then used to fix cells for $15 \mathrm{~min}$ at $4^{\circ} \mathrm{C}$. Finally, cells were pelleted by centrifugation at $500 \mathrm{xg}$ for 8 min and resuspended in FACS buffer for analysis. 500,000 cellular events were collected for each sample using the Miltenyi MACSQuant Analyzer 10 or BD LSRFortessa. Compensation controls were utilized to account for spectral overlap before data analysis was performed using FlowJo v10.4.2.

\section{Cytokine ELISA}

Splenocytes from naïve, adjuvant-treated, or PIV-vaccinated C57BL/6 and CD4 KO mice were restimulated ex vivo with $1 \times 10^{7}$ virulent $C$. burnetii NMI to evaluate IFN- $\gamma$ levels in supernatants by ELISA as previously described (188). Briefly, $5 \times 10^{6}$ total splenocytes from each treatment group were added in duplicate to a 24-well plate in complete medium (RPMI-1640 supplemented with 10\% FBS $+10 \mathrm{mM}$ HEPES buffer $+10 \mathrm{mM}$ non-essential amino acids $+10 \mathrm{mM}$ sodium pyruvate) . Cells were cultured alone or restimulated with $C$. burnetii NMI $\left(1 \times 10^{7} / \mathrm{ml}\right)$ for 3 or 6 days at $37^{\circ} \mathrm{C}$. ELISAs were performed on supernatants for IFN- $\gamma$ as directed by the manufacturer (Thermo Fisher Scientific). 


\begin{tabular}{|c|c|c|c|c|c|}
\hline 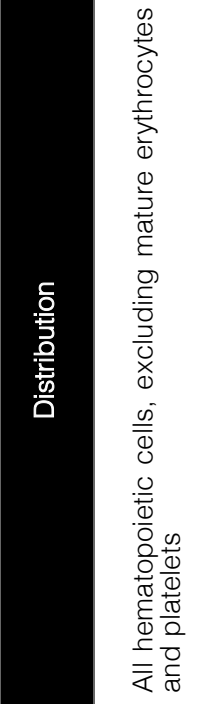 & 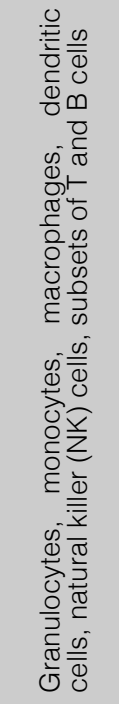 & 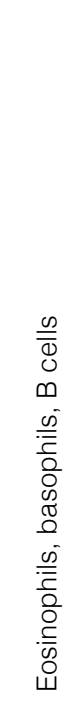 & 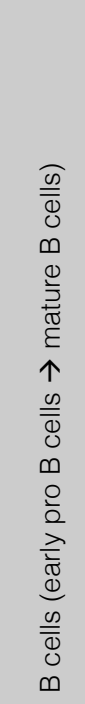 & 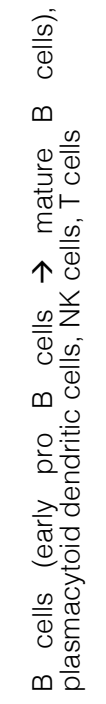 & 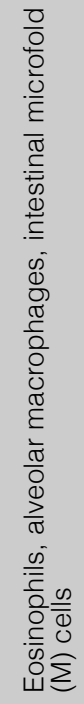 \\
\hline 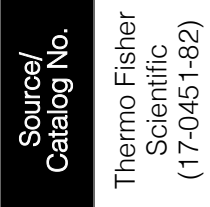 & 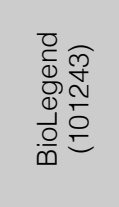 & 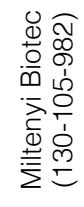 & 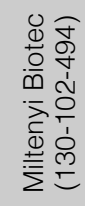 & 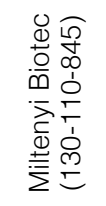 & 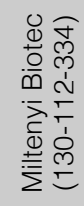 \\
\hline $\begin{array}{l}0 \\
\frac{1}{<}\end{array}$ & 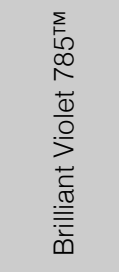 & Ш山 & $\frac{U}{\frac{U}{4}}$ & $\begin{array}{l}\stackrel{\cup}{\square} \\
\frac{E}{\square}\end{array}$ & 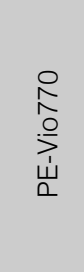 \\
\hline $\begin{array}{l}\frac{F}{5} \\
\text { 交 }\end{array}$ & $\frac{?}{\sum}$ & 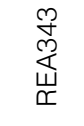 & 苍 & 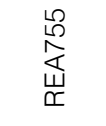 & 离 \\
\hline $\begin{array}{l}\frac{g}{0} \\
\frac{.0}{0} \\
\frac{0}{0} \\
\frac{0}{0} \\
\frac{0}{0} \\
\frac{0}{1}\end{array}$ & $\begin{array}{l}\vec{\pi} \\
\widetilde{\simeq}\end{array}$ & 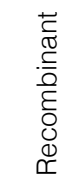 & $\begin{array}{l}\mathbb{\pi} \\
\widetilde{\simeq}\end{array}$ & 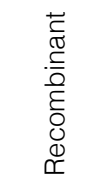 & 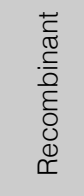 \\
\hline 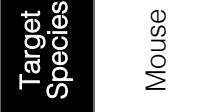 & 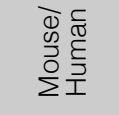 & $\begin{array}{l}0 \\
0 \\
0 \\
\sum \\
\sum\end{array}$ & $\begin{array}{l}0 \\
0 \\
0 \\
\\
\Sigma\end{array}$ & $\begin{array}{l}0 \\
0 \\
0 \\
\sum\end{array}$ & $\begin{array}{l}0 \\
\infty \\
0 \\
\Sigma \\
\Sigma\end{array}$ \\
\hline 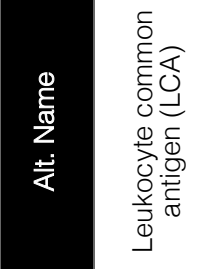 & 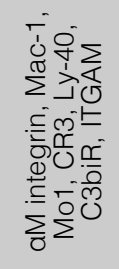 & 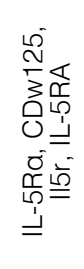 & $\stackrel{+}{\infty}$ & 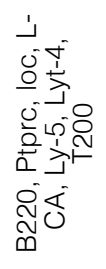 & 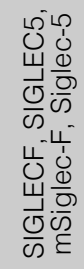 \\
\hline 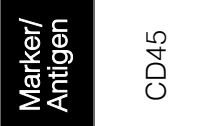 & $\frac{0}{\frac{1}{0}}$ & $\frac{\stackrel{2}{N}}{\stackrel{0}{0}}$ & $\frac{0}{5}$ & $\begin{array}{l}\frac{r}{0} \\
\frac{0}{10} \\
0 \\
0\end{array}$ & 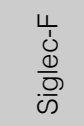 \\
\hline
\end{tabular}




\section{Coxiella-specific ELISA}

Sera from vaccinated and unvaccinated control mice was used for quantification of $\lg M, \lg G$, $\lg G 1$, and $\lg G 2 a$ antibodies. 96-well microtiter plates were coated overnight at $4^{\circ} \mathrm{C}$ with $100 \mu \mathrm{l}$ of inactivated NMI antigen $(0.5 \mu \mathrm{g} / \mathrm{ml})$ or unlabeled anti-lgM or -lgG antibody ( $0.5 \mu \mathrm{g} / \mathrm{ml}$, for the standard curve) (Southern Biotech, Birmingham, $\mathrm{AL}$ ) in $0.05 \mathrm{M}$ carbonate/bicarbonate coating buffer ( $\mathrm{pH}$ 9.6). Plates were then blocked for $1 \mathrm{~h}$ with 1\% BSA in PBS-T buffer $(0.05 \%$ Tween-20 in $1 \mathrm{X}$ PBS), followed by incubation with $200 \mu \mathrm{l}$ of diluted sample serum (1:200-1:1200) or serially-diluted pure $\lg \mathrm{M}, \lg \mathrm{G}, \lg \mathrm{G} 1$, or $\lg \mathrm{G} 2 \mathrm{a}$ (Southern Biotech) for $2 \mathrm{~h}$ at room temp. Plates were washed 4 times with PBS-T buffer and then incubated with 100 $\mu$ of diluted HRP-conjugated goat anti-mouse $\lg M$, $\lg G$, $\lg G 1$, or $\lg G 2 a(1: 4000-$ 1:8000) at room temp for $1 \mathrm{~h}$. Plates were again washed 4 times with PBS-T, followed by the addition of $100 \mu \mathrm{l}$ TMB substrate (Thermo Fisher Scientific). Reactions were stopped using $1 \mathrm{M} \mathrm{H}_{3} \mathrm{PO}_{4}$ and absorbance was measured at 450 nm using the SpectraMax ${ }^{\circledR}$ (Molecular Devices, San Jose, CA) or Infinite F50 (Tecan, Switzerland) microplate reader.

\section{Adoptive cell transfer}

Spleen cells were harvested from naïve and vaccinated WT C57BL/6 mice for adoptive cell transfer at $28 \mathrm{dpv}$. To obtain purified $\mathrm{CD} 4^{+} \mathrm{T}$ cells, magnetic cell separation was performed using a negative isolation kit (Miltenyi Biotec, Germany). 
Naïve and vaccinated CD4 KO mice received $5 \times 10^{6}$ purified $\mathrm{CD}^{+} \mathrm{T}$ cells via i.p. injection 24 hours before infection.

\section{Histopathology}

Spleens were fixed in $10 \%$ formalin for $48 \mathrm{~h}$ before processing by the University of Missouri Veterinary Medicine Diagnostic Laboratory (MU-VMDL). Briefly, tissues were embedded in paraffin, sectioned, and stained with hematoxylin and eosin. Spleen sections were then scored for histiocytic inflammation in red pulp following blinded review by a trained pathologist. Pathology scores were assigned based on the following scale: 0 , no accumulations of macrophages; 1 , small accumulations of macrophages; 2 , small to moderate accumulations of macrophages; and 3, moderate to large accumulations of macrophages.

\section{Statistical analysis}

Prism 5.0 (GraphPad Software Inc., San Diego, CA) was used for all statistical analyses. Results represent the mean \pm standard deviation. Parametric data were compared using t-test, one-way ANOVA, or two-way ANOVA with Sidak's, Tukey's, or Dunnett's multiple comparisons test. Nonparametric data were compared using Kruskal-Wallis with Dunn's multiple comparisons test. Differences were significant at $p<0.05$. 


\section{CHAPTER 3: EOSINOPHILS AFFECT ANTIBODY ISOTYPE SWITCHING AND MAY PARTIALLY CONTRIBUTE TO EARLY VACCINE-INDUCED IMMUNITY AGAINST COXIELLA BURNETII}

\section{Abstract}

Coxiella burnetii is an obligate intracellular Gram-negative bacterium that causes human $Q$ fever. An acidified citrate cysteine medium (ACCM-2) has been developed which mimics the intracellular replicative niche of $C$. burnetii and allows axenic growth of the bacteria. To determine if C. burnetii cultured in ACCM-2 retains immunogenicity, we compared the protective efficacy of PIV and PIIV derived from axenic culture 7, 14, and $28 \mathrm{dpv}$. PIV conferred significant protection against virulent $C$. burnetii as early as $7 \mathrm{dpv}$, which suggests ACCM-2-derived PIV retains immunogenicity and protectivity. We analyzed the cellular immune response in spleens from PIV- and PIIV-vaccinated mice by flow cytometry 7 and $14 \mathrm{dpv}$ and found significantly more granulocytes in PIV-vaccinated mice compared to PIIV-vaccinated mice. Interestingly, we found these infiltrating granulocytes to be $S S C^{\text {high }} \mathrm{CD} 11 \mathrm{~b}^{+} \mathrm{CD} 125^{+} \mathrm{Siglec}-\mathrm{F}^{+}$eosinophils. There was no change in the number of eosinophils in PIV-vaccinated CD4 KO mice compared to controls, which suggests that eosinophil accumulation is $\mathrm{CD}^{+}{ }^{+} \mathrm{T}$ cell-dependent. To evaluate the importance of eosinophils in PIV-mediated protection, we vaccinated and challenged eosinophil-deficient $\triangle \mathrm{dbIGATA}$ mice. $\triangle \mathrm{db}$ IGATA mice had significantly worse disease than their WT counterparts when challenged $7 \mathrm{dpv}$, 
while no significant difference was seen $28 \mathrm{dpv}$. Nevertheless, $\triangle \mathrm{dbIGATA}$ mice had elevated serum IgM with decreased $\lg G 1$ and $\lg G 2 a$ whether mice were challenged 7 or $28 \mathrm{dpv}$. These results suggest that eosinophils may play a role in early vaccine protection against $C$. burnetii and contribute to antibody isotype switching.

\section{Introduction}

Previous work suggests that both humoral and $\mathrm{CMI}$ are involved in vaccine protection against $C$. burnetii $(117,119,137,174,189)$, although the contribution of innate immunity is unclear. The innate immune system is our first line of defense against infection and becomes activated through sensing of pathogen-associated molecular patterns (PAMPs) by pattern recognition receptors (PRRs) on or within innate cells. Distinct PRRs recognize different microbial components (e.g. bacterial lipopeptides, LPS), eliciting unique downstream signaling events. Innate cells integrate these signals and translate them into a transcriptional program tailored to the specific microbe. This involves the upregulation of inflammatory genes encoding cytokines, chemokines, and costimulatory molecules which directly influence adaptive immunity. As such, the innate immune system is a useful tool that can be manipulated to enhance vaccine protection. In fact, the use of Toll-like receptor (TLR) agonists as immunoadjuvants has proved effective when incorporated into vaccines against multiple pathogens (190-195).

Here, we use the recently developed host-cell free culture system (196) and assess the immunogenicity of PIV and PIIV. The data indicate that PIV elicits 
superior protection compared to PIIV at all time points examined. Furthermore, using flow cytometry to examine the cellular immune response, we find that PIV and PIIV differ in the accumulation of eosinophils in the spleen. This accumulation appears to be $\mathrm{CD}^{+} \mathrm{T}$ cell-dependent, as PIV-vaccinated CD4 KO mice do not have elevated eosinophils in their spleens. Increased splenomegaly and bacterial burden in PIV-vaccinated eosinophil-deficient $\Delta$ dbIGATA mice compared to WT mice challenged $7 \mathrm{dpv}$ suggests a partial role for eosinophils in early vaccine protection. Additionally, elevated serum IgM coupled with decreased IgG1 and IgG2a subclass antibodies in $\triangle$ dblGATA mice suggests a role for these cells in antibody isotype switching. These findings highlight the utility of the axenic culture system for the manufacture of $C$. burnetii vaccines and provide new insights into the contribution of innate immunity to vaccine protection against $C$. burnetii, which can be applied to $Q$ fever vaccine development.

\section{Results}

\section{Host cell-free $C$. burnetii retains immunogenicity and protectivity}

Q-VAX ${ }^{\circledR}$ is produced in embryonated eggs (197) and, while this allows for the generation of large quantities of material at a relatively low cost, residual egg proteins often remain in the final vaccine preparation and contribute to the high incidence of vaccine reactions (132). ACCM-2 is a medium that mimics the intracellular replicative niche of $C$. burnetii and allows axenic growth of the bacteria. This medium removes the potential for host contaminants while still generating large quantities of material in a short time ( $\sim 3$ logs of growth in 6 days) 
(177). Previous reports have shown that $C$. burnetii strains grown in ACCM-2 as well as a newer formulation, ACCM-D, retain in vivo infectivity in mouse and guinea pig models $(101,177)$. To determine if ACCM-2-derived $C$. burnetii retains immunogenicity, we vaccinated BALB/c mice s.c. with $10 \mu \mathrm{g}$ PIV or PIIV with Alhydroge $\left.\right|^{\circledR}$ adjuvant and challenged i.p. with $5 \times 10^{7}$ C. burnetii NMI 28 days later. An aluminum hydroxide adjuvant was selected for these studies due to their broadly accepted use in FDA-approved vaccines for humans (198). To assess disease severity, we evaluated splenomegaly and bacterial burden in the spleen $14 \mathrm{dpi}$. Similar to previously reported vaccines generated from chicken eggs or cell culture (117), we found that PIV grown in ACCM-2 significantly reduces splenomegaly compared to PIIV and adjuvant controls (Figure 3-1A). Additionally, we found that bacterial burden in the spleens of PIV-vaccinated animals was reduced compared to PIIV and adjuvant controls (Figure 3-1B). These results indicate that axenically-generated PIV retains immunogenicity and protectivity.

\section{PIV protects BALB/c mice as early as 1 week post-vaccination}

While it has been established that PIV is protective $28 \mathrm{dpv}$, it remains unknown how early mice are protected from developing clinical disease following vaccination. To test this, we vaccinated BALB/c mice as described previously and then challenged 7 or $14 \mathrm{dpv}$ with $1 \times 10^{7}$ C. burnetii NMI by i.p. injection. Due to the high degree of splenomegaly ( 8\%) observed with a challenge dose of $5 \times 10^{7}$ (Figure $3-1 \mathrm{~A}$ ), we lowered the challenge dose to $1 \times 10^{7}$ for all subsequent infections. PIV had significantly reduced splenomegaly compared to PIIV and 
A

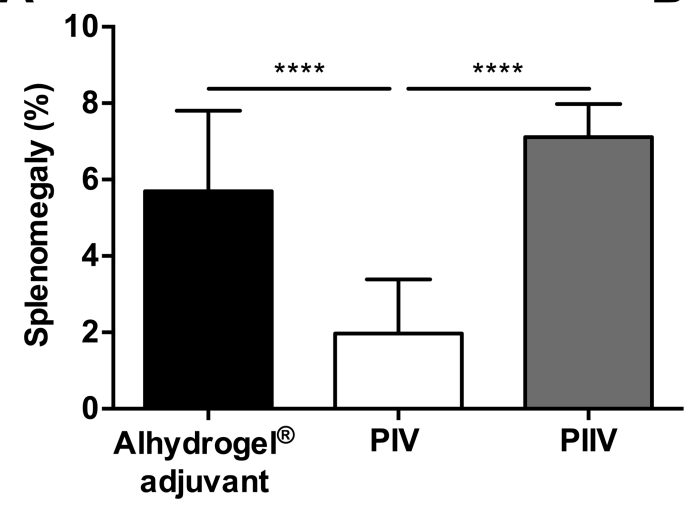

B

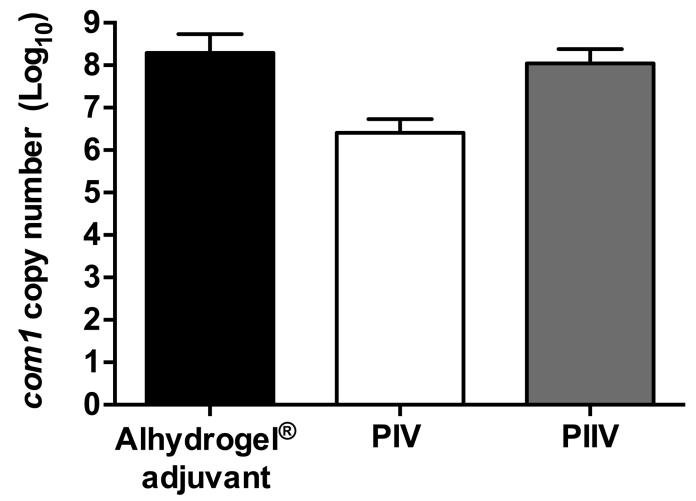

Figure 3-1. Host cell-free $C$. burnetii retains immunogenicity and protectivity

BALB/c mice were vaccinated s.c. with $10 \mu \mathrm{g}$ of PIV or PIIV and challenged via i.p. route with $5 \times 10^{7}$ C. burnetii NMI bacteria at $28 \mathrm{dpv}$. Mice receiving Alhydrogel ${ }^{\circledR}$ adjuvant alone served as unvaccinated controls. Splenomegaly (A) and bacterial burden in the spleen (B) were evaluated at $14 \mathrm{dpi}$ to compare protective efficacies. Results are expressed as percent splenomegaly [(spleen weight/body weight)x100]. Bacterial burden was determined by qPCR and is expressed as $\log _{10}$ C. burnetii com1 gene copy numbers. Each experimental group includes four mice, with error bars representing the standard deviations from the means. ${ }^{* \star * *}, \mathrm{p}<0.0001$, as determined by one-way ANOVA with Tukey's multiple-comparison test. 


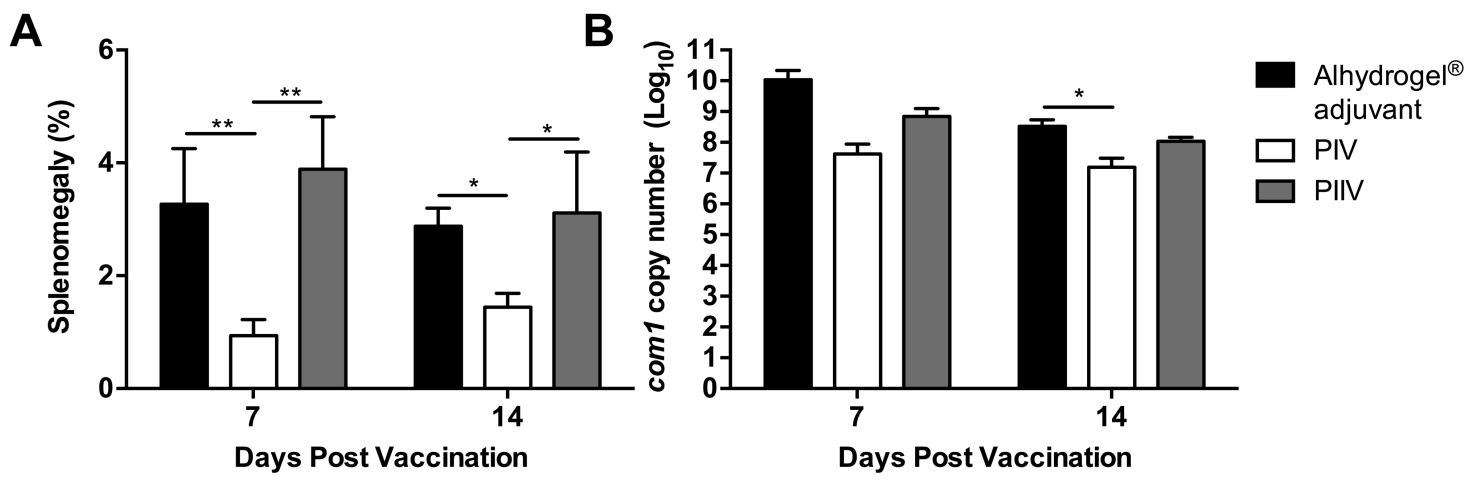

Figure 3-2. PIV protects BALB/c mice as early as 1 week post-vaccination

BALB/c mice were vaccinated s.c. with $10 \mu \mathrm{g}$ of PIV or PIIV and challenged via i.p. route with $1 \times 10^{7}$ C. burnetii NMl bacteria at 7 or $14 \mathrm{dpv}$. Mice receiving Alhydroge ${ }^{\circledR}$ adjuvant alone served as unvaccinated controls. Splenomegaly (A) and bacterial burden in the spleen (B) were evaluated at $14 \mathrm{dpi}$ to compare protective efficacies. Results are expressed as percent splenomegaly [(spleen weight/body weight)x100]. Bacterial burden was determined by qPCR and is expressed as $\log _{10} C$. burnetii com1 gene copy numbers. Each experimental group includes four mice, with error bars representing the standard deviations from the means. ${ }^{*}, p<0.05 ;{ }^{* *}$, $\mathrm{p}<0.01$, as determined by one-way ANOVA with Tukey's multiple-comparison test. 
adjuvant controls when challenged 7 or $14 \mathrm{dpv}$ (Figure 3-2A). Additionally, mice vaccinated with PIV had a reduction in splenic bacterial burden at all time points examined (Figure 3-2B). Taken together, these data suggest that immunity develops as early as 1 week post-vaccination.

\section{PIV significantly increases side scatter high granulocytes in the spleen}

PIV significantly reduces splenomegaly and bacterial burden in BALB/c mice challenged with virulent $C$. burnetii NMI. However, PIIV fails to protect mice from clinical disease. The immunological differences between PIV and PIIV that lead to such disparate outcomes remain unknown. Considering the early time point at which this difference becomes apparent $(\sim 7 \mathrm{dpv})$, we hypothesized that innate cells may be differentially recruited to the spleen following vaccination. To test this hypothesis, we used flow cytometry to analyze cellular recruitment to the spleens of adjuvant control, PIV- or PIIV-vaccinated BALB/c mice 7 and 14 dpv. Naïve animals were used to establish baseline values for all populations examined. Figure 3-3A details the gating strategy used for the initial analysis. Cellular debris and doublets were removed before gating on lymphocytes, monocytes, and granulocytes based on their forward (FSC) and side scatter (SSC) characteristics. The frequency of lymphocytes was not significantly altered at either time point, though there was a significant increase in the number of lymphocytes in PIVvaccinated mice compared to adjuvant controls $14 \mathrm{dpv}$ (Figure 3-3B). While there were significant changes in the frequency of monocytes in PIV-vaccinated mice compared to PIIV and adjuvant controls $14 \mathrm{dpv}$, there were no significant 
differences in the number of monocytes at either time point (Figure 3-3C). However, there was a significant increase in the frequency of SSChigh granulocytes in PIV-vaccinated mice compared to PIIV and adjuvant controls $7 \mathrm{dpv}$. Additionally, the total number of granulocytes in the spleen was significantly higher in PIVvaccinated mice compared to PIIV and adjuvant controls (Figure 3-3D). Overall, these data demonstrate that PIV elicits granulocyte accumulation in the spleen early in the vaccine response.

\section{SSC $^{\text {high }}$ granulocytes express CD11b and the interleukin-5 receptor}

Following the observation that PIV elicits significant accumulation of granulocytes in the spleen, we further analyzed this population to determine which granulocytic cells were responding. Figure 3-4A details the gating strategy used for analysis. Cellular debris and doublets were removed as previously described. When compared to PIIV and adjuvant controls, PIV-vaccinated animals had a significantly higher frequency of SSChigh cells that expressed CD11b (Figure 3-4B). Furthermore, the total number of SSChigh CD11 $\mathrm{b}^{+}$cells in the spleens of PIVvaccinated animals was significantly higher than PIIV or adjuvant controls.

CD11 $\mathrm{b}^{+}$granulocytic cells include neutrophils and eosinophils. To distinguish these groups, we next looked at interleukin-5 receptor alpha subunit expression (IL-5Ra, CD125). CD125 is expressed on eosinophils, but not on neutrophils (199). Interestingly, PIV-vaccinated mice had significantly more CD125+ cells than PIIV or adjuvant controls (Figure 3-4C). These results suggest that the granulocytes recruited to the spleens of PIV-vaccinated mice may be eosinophils. 

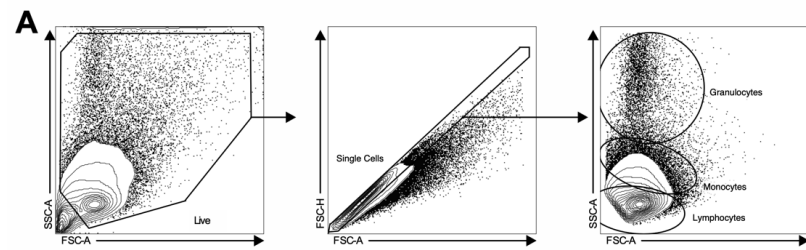

B

Lymphocytes

C
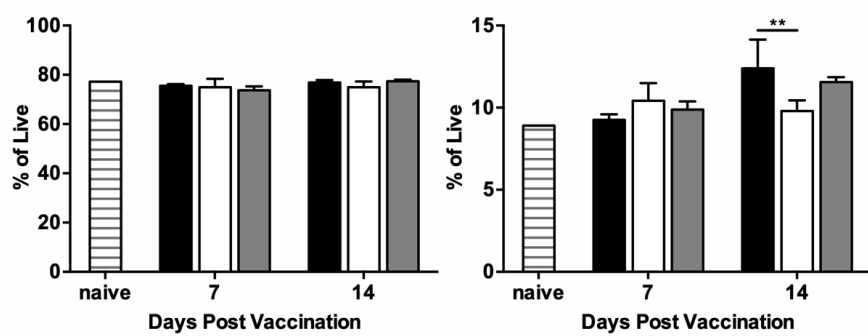

D Granulocytes
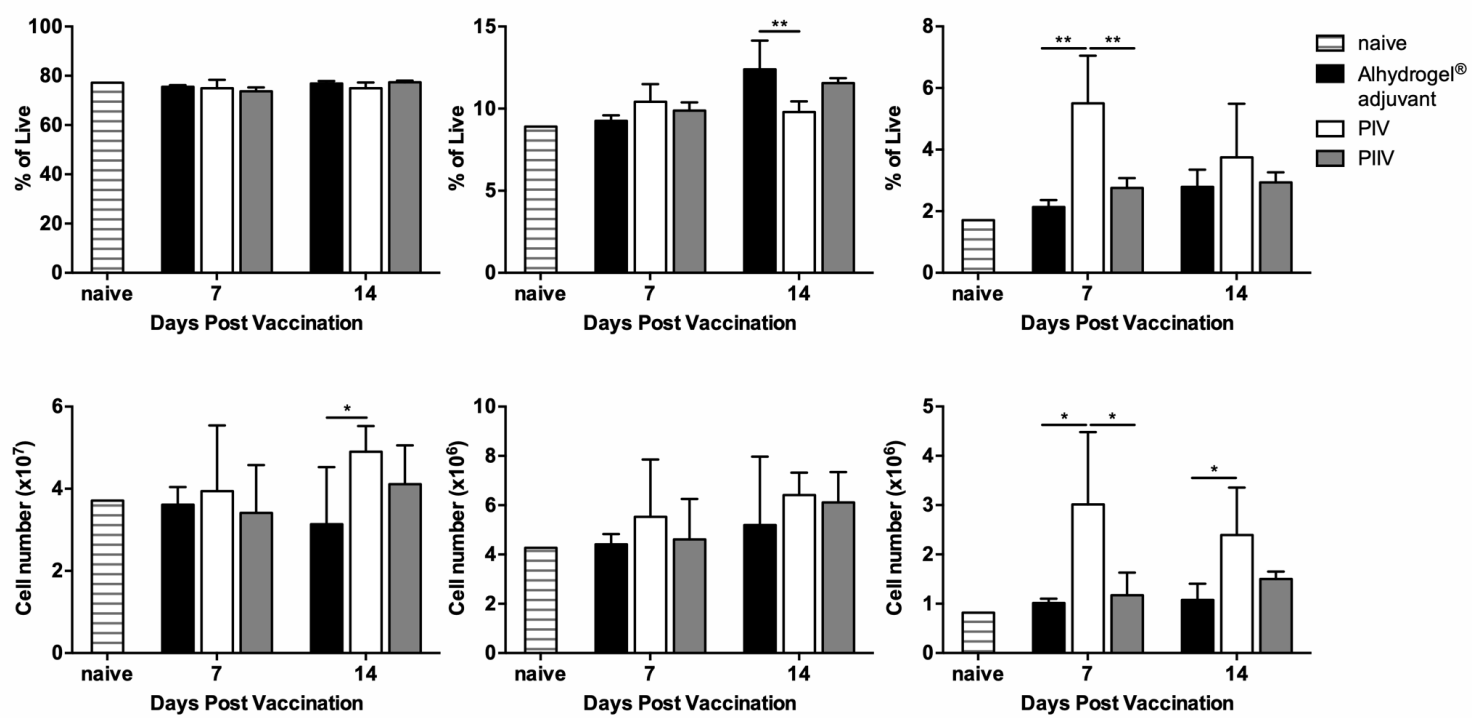

Figure 3-3. PIV significantly increases high side scatter granulocytes in the spleen

BALB/c mice were vaccinated s.c. with $10 \mu \mathrm{g}$ of PIV or PIIV, and spleens were processed for flow cytometry as described in Materials and Methods. Mice receiving Alhydrogel ${ }^{\circledR}$ adjuvant alone served as unvaccinated controls. Naïve mice were used to establish baseline values. (A) Gating strategy. Forward (FSC) and side scatter (SSC) were used to identify lymphocytes, monocytes, and granulocytes as well as to remove cell debris from the analysis. (B to D) Population frequencies and absolute cell counts were determined 7 and $14 \mathrm{dpv}$. Each experimental group includes 4 to 6 mice, with error bars representing the standard deviations from the means. *, $p<0.05 ;{ }^{* \star}, p<0.01$, as determined by one-way ANOVA with Tukey's multiple-comparison test. 

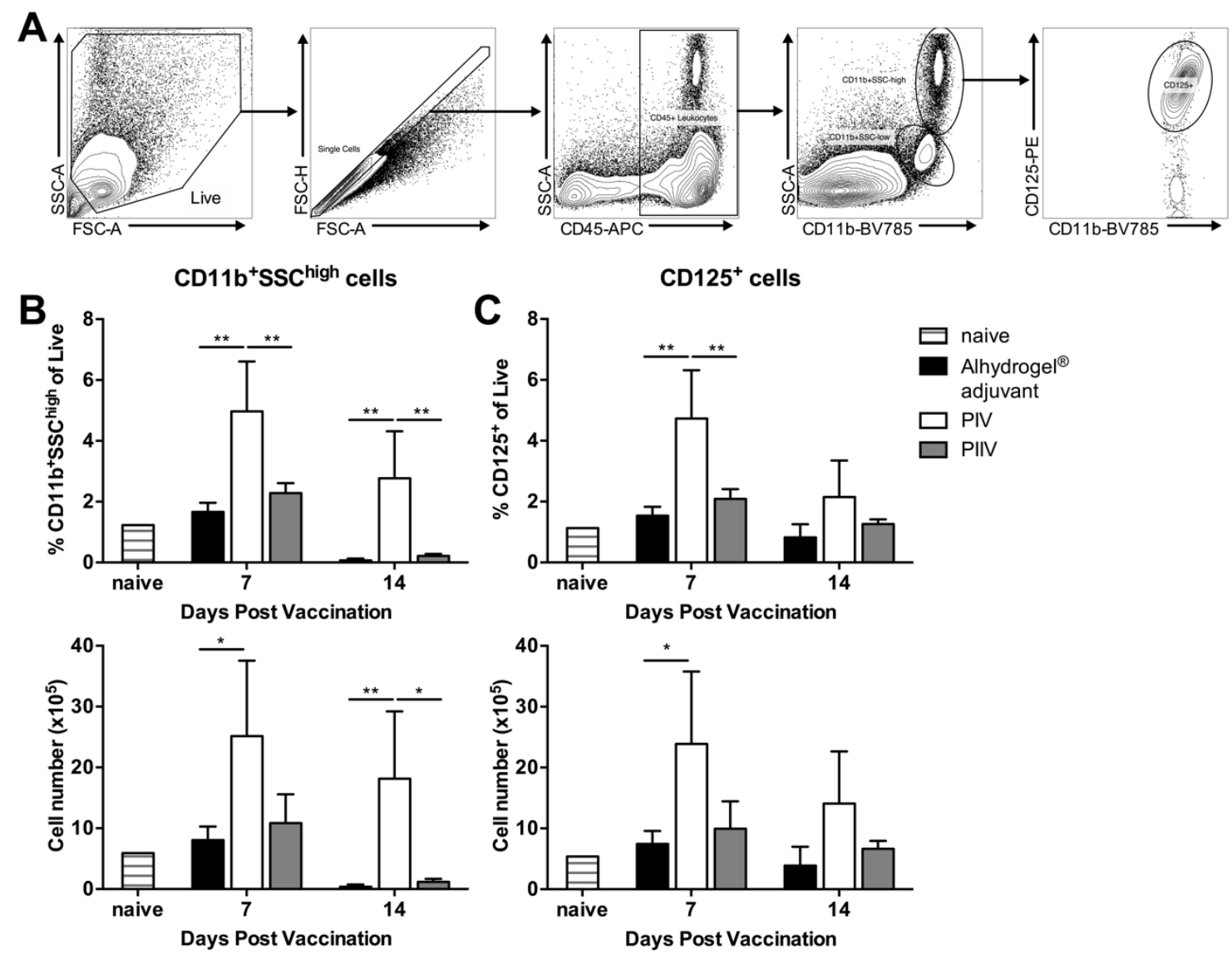

Figure 3-4. SSC ${ }^{\text {high }}$ granulocytes express CD11b and the interleukin-5 receptor

BALB/c mice were vaccinated s.c. with $10 \mu \mathrm{g}$ of PIV or PIIV, and spleens were processed for flow cytometry as previously described. Mice receiving Alhydroge ${ }^{\circledR}$ adjuvant alone served as unvaccinated controls. Naïve mice were used to establish baseline values. (A) Gating strategy. FSC and SSC were used to remove cell debris from the analysis. CD45+ leukocytes were then identified and further subgated based on SSC and expression of CD11b. CD11b+SSChigh granulocytes were then further subgated based on expression of the interleukin-5 receptor alpha subunit (IL-5R $\alpha, C D 125)$. (B and C) Population frequencies and absolute cell counts were determined 7 and $14 \mathrm{dpv}$. Each experimental group includes 4 to 6 mice, with error bars representing the standard deviations from the means. ${ }^{*}, p<0.05 ;{ }^{*}, p<0.01$, as determined by one-way ANOVA with Tukey's multiple-comparison test. 


\section{SSC ${ }^{\text {high }}{ }^{C D 11 b+C D 125}+$ cells are Siglec-F+ eosinophils}

While CD125 is most commonly expressed on eosinophils, there are subsets of B1 cells which also express CD125 (200). To determine the phenotype of these cells, we evaluated their expression of Siglec-F, an eosinophil specific marker, as well as CD19/B220 (Figure 3-5A). When compared to PIIV and adjuvant controls, PIV-vaccinated mice had significantly more Siglec- $\mathrm{F}^{+}$positive cells $7 \mathrm{dpv}$ (Figure 3-5B). Less than $0.1 \%$ of the SSC high CD $11 b^{+} C D 125^{+}$cells were CD19/B220+ (Figure 3-5C). Taken together, this data indicates that PIV and PIIV differ in the early recruitment of eosinophils to the spleen.

\section{Eosinophil accumulation in PIV-vaccinated mice is $\mathrm{CD}^{+} \mathbf{T}$ cell-dependent}

CD4+ $T$ cells are potent producers of IL-5, which promotes eosinophil differentiation, maturation, migration, and activation $(202,203)$. Therefore, to determine if eosinophil accumulation in PIV-vaccinated spleens is dependent on signals from CD4+ T cells, we vaccinated CD4 KO mice with $10 \mu \mathrm{g}$ PIV and assessed eosinophil numbers 7 days later. Interestingly, we observed a defect in eosinophil accumulation in PIV-vaccinated CD4 KO mice compared to PIVvaccinated WT mice (Figure 3-6A). There was no difference in the number of eosinophils between naïve WT and CD4 KO mice, which indicates that this phenomenon was not due to an inherent defect in CD4 KO mice. Overall, this data suggests that eosinophil accumulation in PIV-vaccinated mice is $\mathrm{CD}^{+} \mathrm{T}$ celldependent.

Next, we assessed whether the loss of eosinophils in CD4 KO mice had any effect 

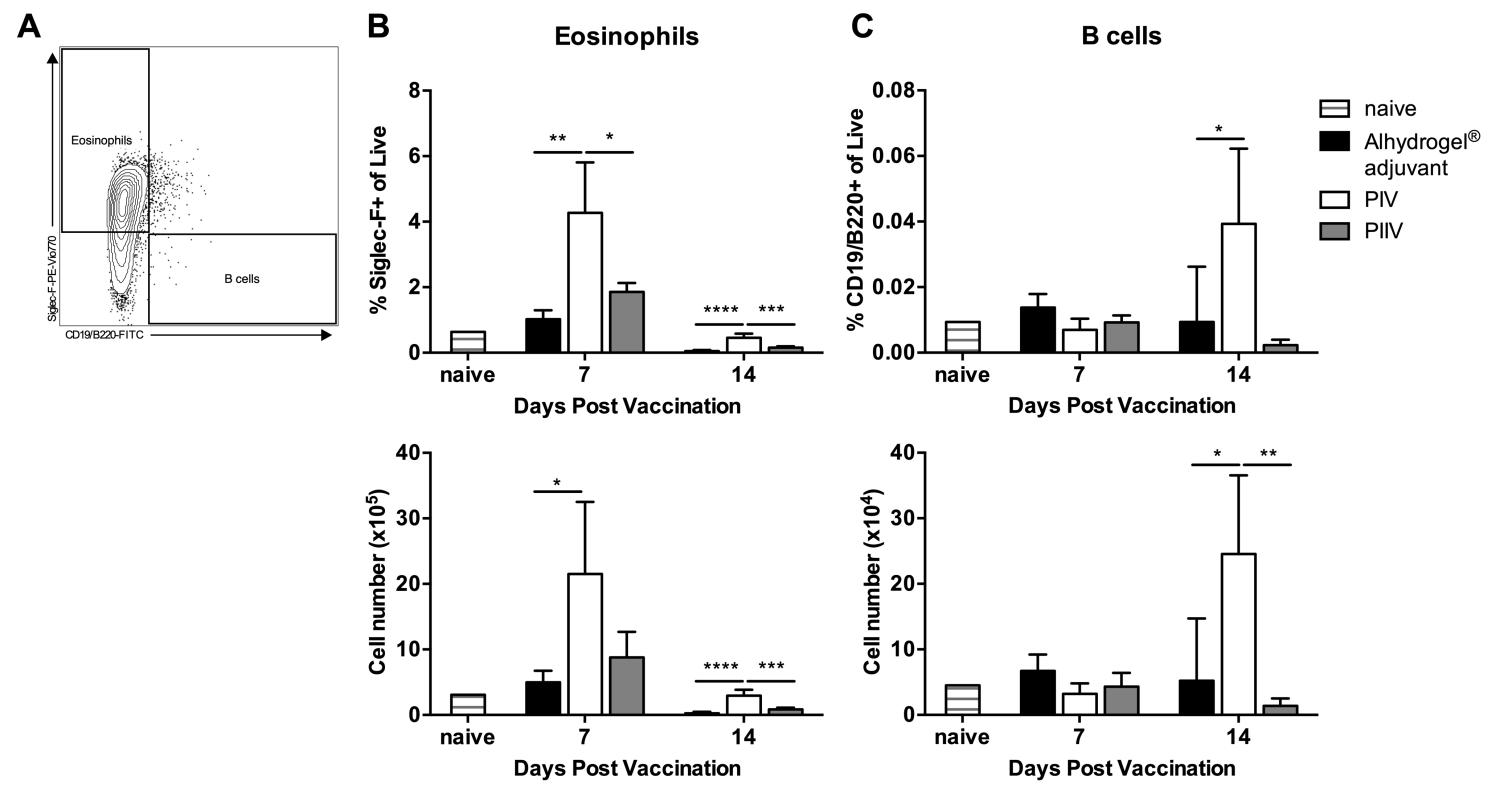

Figure 3-5. SSC ${ }^{\text {high }} \mathrm{CD} 11 \mathrm{~b}+\mathrm{CD} 125^{+}$cells are Siglec- $\mathrm{F}^{+}$eosinophils

BALB/c mice were vaccinated s.c. with $10 \mu \mathrm{g}$ of PIV or PIIV, and spleens were processed for flow cytometry. Mice receiving Alhydroge $\left.\right|^{\circledR}$ adjuvant alone served as unvaccinated controls. Naïve mice were used to establish baseline values. (A) Gating strategy. SSChigh CD11b+CD125+ cells were examined for expression of Siglec-F (eosinophils) and CD19/B220 (B cells). (B and C) Population frequencies and absolute cell counts were determined 7 and $14 \mathrm{dpv}$. Each experimental group includes 4 to 6 mice, with error bars representing the standard deviations from the means. ${ }^{*}, \mathrm{p}<0.05 ;{ }^{* *}, \mathrm{p}<0.01 ;{ }^{* * *}, \mathrm{p}<0.001 ;{ }^{* * *}, \mathrm{p}<0.0001$, as determined by one-way ANOVA with Tukey's multiple-comparison test. 
on the production of IFN- $\gamma$, an essential cytokine for $C$. burnetii clearance (201). To test this, we isolated total splenocytes from naïve, adjuvant-treated and PIVvaccinated WT and CD4 KO mice for ex vivo restimulation as described previously (188). IFN- $\gamma$ levels were measured by ELISA 3 and 6 days post-restimulation with either media alone or $1 \times 10^{7}$ C. burnetii NMI. Splenocytes from PIV-vaccinated WT mice produced significant levels of IFN- $\gamma$ in response to $C$. burnetii NMI at all time points (Figure 3-6B). However, while PIV-vaccinated CD4 KO mice were still able to respond to $C$. burnetii $\mathrm{NMI}$, there was a significant defect in their production of IFN- $\gamma$ both 3 and 6 days post-restimulation compared to PIV-vaccinated WT mice. This data suggests a CD4-dependent mechanism of IFN- $\gamma$ production which could be partially related to the absence of significant eosinophil accumulation.

\section{Eosinophils may partially contribute to early PIV protection}

Following the observation that eosinophils are recruited to the spleens of PIVvaccinated mice, we evaluated whether these cells were important for vaccinemediated protection. To test this, we vaccinated eosinophil-deficient $\triangle$ dbIGATA mice with $10 \mu \mathrm{g}$ PIV and challenged i.p. with $1 \times 10^{7}$ C. burnetii NMI 7 days later. Disease severity was evaluated by comparing body weight loss, splenomegaly, and bacterial burden $14 \mathrm{dpi}$. Compared to WT PIV-vaccinated mice, $\triangle \mathrm{dbIGATA}$ mice displayed no significant loss in body weight throughout infection (Figure 37A). However, $\triangle$ dbIGATA mice had significantly higher splenomegaly (Figure 37B) and bacterial burden (Figure 3-7C) compared to their WT PIV-vaccinated counterparts. These data indicate that eosinophils may partially contribute to the 


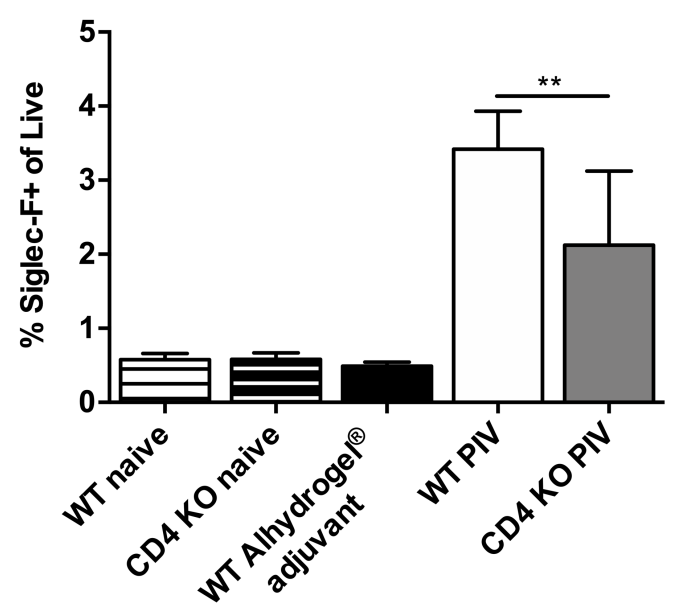

B

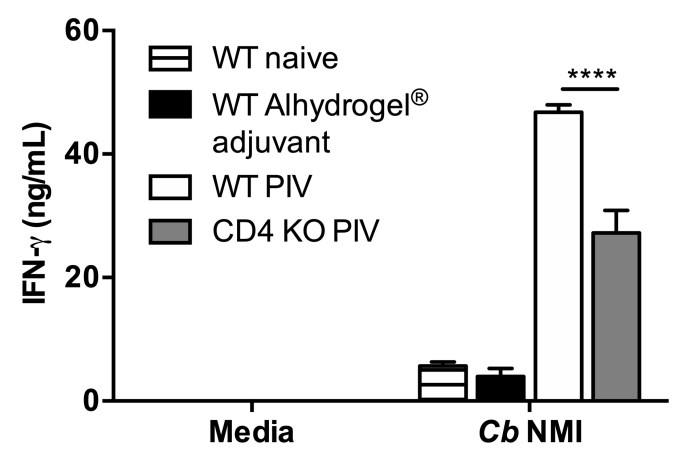

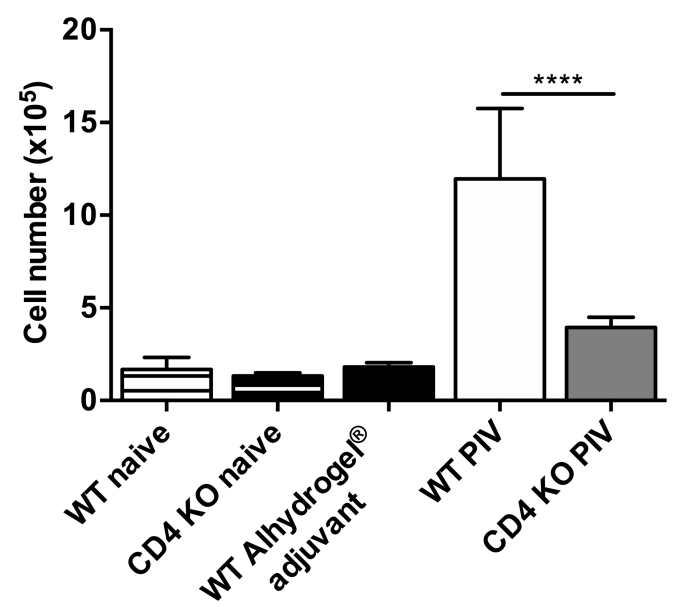

6 days

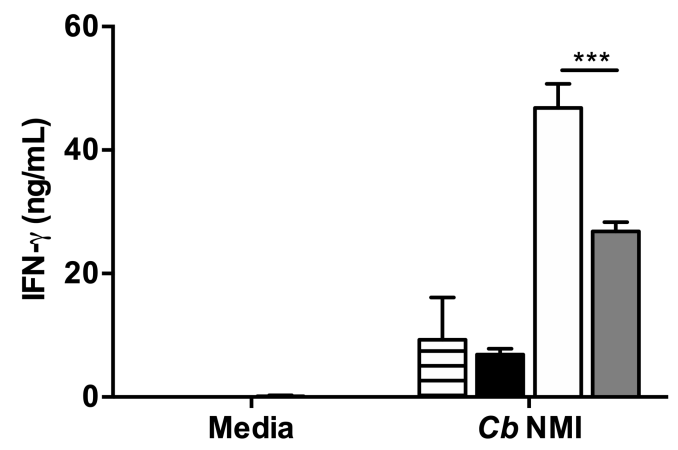

Figure 3-6. Eosinophil accumulation in PIV-vaccinated mice is $\mathrm{CD} 4^{+} \mathrm{T}$ cell dependent

WT C57BL/6 and CD4-deficient mice were vaccinated s.c. with $10 \mu \mathrm{g}$ of PIV, and 7 days later spleens were harvested for flow cytometry or ex vivo restimulation studies. Mice receiving Alhydroge ${ }^{\circledR}$ adjuvant alone served as unvaccinated controls. Naiive mice were used to establish baseline values. (A) SSC high $\mathrm{CD} 11 \mathrm{~b}^{+} \mathrm{CD} 125^{+}$Siglec- $\mathrm{F}^{+}$eosinophils were enumerated using a previously described gating strategy. (B) Total splenocytes were isolated and restimulated with medium or with $1 \times 10^{7} \mathrm{C}$. burnetii NMI bacteria for 3 and 6 days, and cell culture supernatants were evaluated for IFN- $\gamma$ production by cytokine-specific ELISA. Each experimental group includes 5 mice, with error bars representing the standard deviations from the means. ${ }^{* *}, p<0.01$; ${ }^{* \star *}, p<0.001 ;{ }^{* \star * \star}, p<0.0001$, as determined by one-way ANOVA with Tukey's multiple-comparison test. $C b, C$. burnetii, $\mathrm{KO}$, knockout. 
early vaccine response against $C$. burnetii infection.

Eosinophils are known to play a role in priming the early lgM response as well as eliciting Th2 cells through the secretion of cytokines (202). To evaluate the effect of eosinophil deficiency on antibody-mediated immunity, we measured $C$. burnetii NMI-specific IgM and IgG by ELISA. At $7 \mathrm{dpv}$, there was significantly more NMIspecific serum IgM in $\triangle \mathrm{dbIGATA}$ mice compared to their WT counterparts (Figure 3-7D). At this time point, however, there was no significant difference in IgG.

To evaluate the secondary response, we challenged $\triangle \mathrm{dbIGATA}$ mice $7 \mathrm{dpv}$ and again measured NMI-specific IgM and IgG by ELISA. At $14 \mathrm{dpi}$, there was a significant increase in the serum concentration of NMI-specific IgM in $\triangle \mathrm{dbIGATA}$ mice (Figure 3-7E) compared to WT. Again, no significant difference in IgG was measured between PIV-vaccinated cohorts. However, while there was not a significant difference in total IgG, there were significant decreases in IgG subclasses associated with eosinophil deficiency. Specifically, there was significantly less NMI-specific IgG1 and IgG2a in $\triangle \mathrm{dblGATA}$ mice compared to their WT counterparts (Figure 3-7E). Collectively, these data suggest that eosinophils may partially contribute to the early vaccine response as well as antibody isotype switching.

\section{Eosinophils contribute to antibody isotype switching in late PIV responses}

Next, we examined whether eosinophils also contribute to the late PIV response. WT and $\triangle \mathrm{dbIGATA}$ mice were vaccinated as previously described and challenged i.p. with $1 \times 10^{7}$ C. burnetii NMI 28 days later. Body weight loss, splenomegaly, and 
A

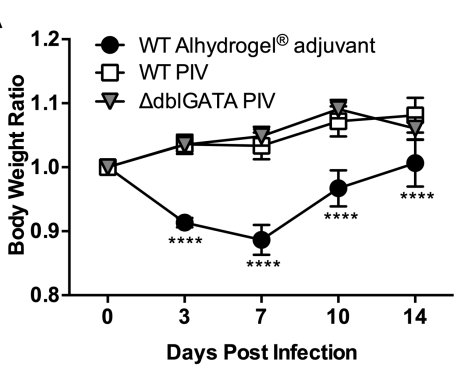

D
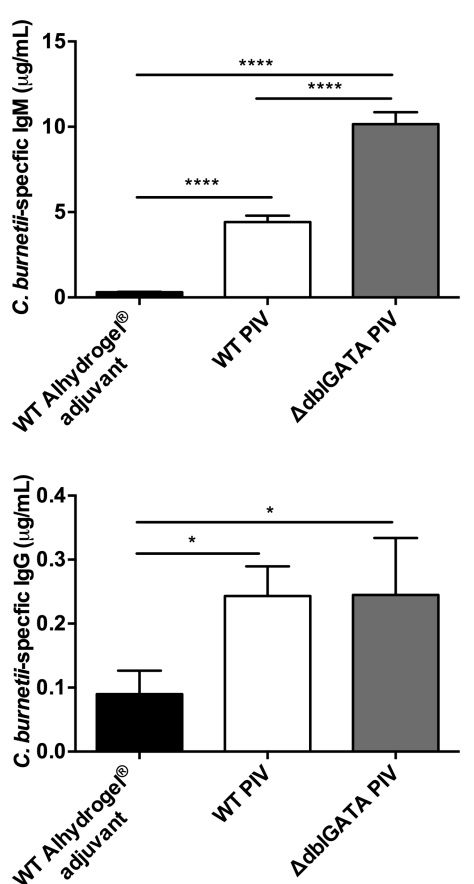

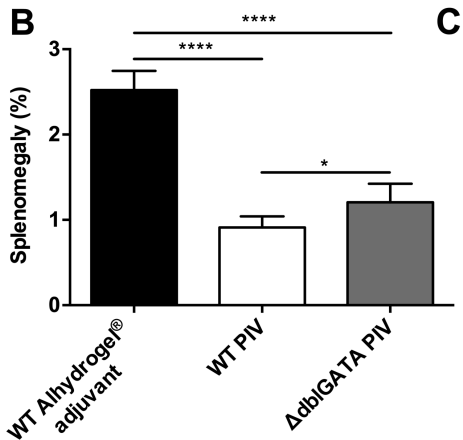

C

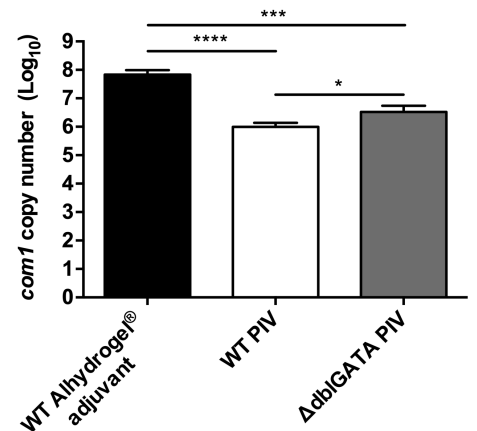

E

Post-challenge
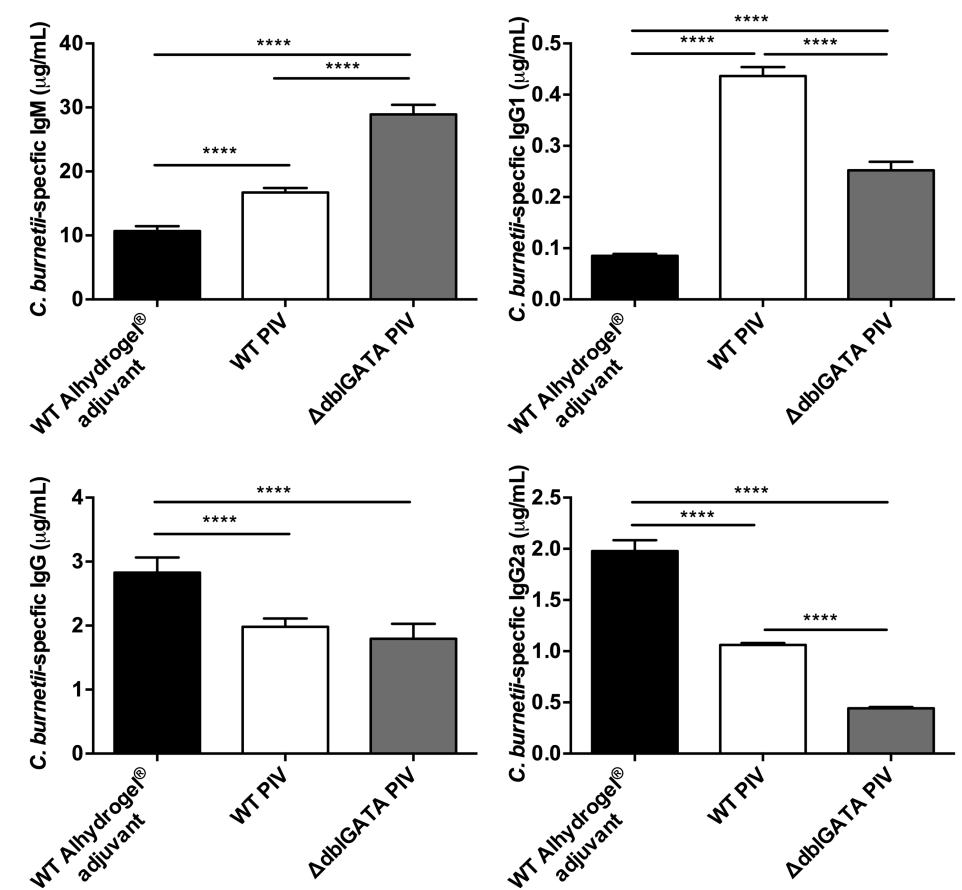

Figure 3-7. Eosinophils play a role in early PIV protection

WT and eosinophil-deficient $\Delta$ dbIGATA mice were vaccinated s.c. with $10 \mu \mathrm{g}$ of PIV 7 days prior to i.p. challenge with $1 \times 10^{7} \mathrm{C}$. burnetii NMI bacteria. (A) Relative body weight was measured throughout infection. Splenomegaly (B) and bacterial burden in the spleen (C) were evaluated at 14 dpi to assess disease severity. C. burnetii NMI-specific IgM and IgG levels were evaluated 7 dpv (D) or 14 dpi (E). Results are expressed as percent splenomegaly [(spleen weight/body weight)x100]. Bacterial burden was determined by $\mathrm{qPCR}$ and is expressed as $\log _{10} C$. burnetii com1 gene copy numbers. Each experimental group includes 5 mice, with error bars representing the standard deviations for the means. ${ }^{*}, p<0.05 ;{ }^{* \star *}, p<0.001 ;{ }^{* * *}, p<0.0001$, as determined by two-way ANOVA with Dunnett's multiple-comparison test (for panel A) or by one-way ANOVA with Tukey's multiple-comparison test (for panels B to E). 
bacterial burden were examined to assess disease severity $14 \mathrm{dpi}$. No body weight loss was observed in the $\triangle$ dbIGATA cohort compared to WT (Figure 3-8A). Additionally, there was no significant difference in splenomegaly (Figure 3-8B) or bacterial burden (Figure 3-8C) between PIV-vaccinated WT and $\Delta$ dbIGATA mice. These results suggest that eosinophils may not play a role in later protection. To determine if eosinophils play a role in the IFN- $\gamma$ response, we measured IFN- $\gamma$ in serum from PIV-vaccinated WT and $\triangle \mathrm{dbIGATA}$ mice by ELISA. Interestingly, there was significantly less IFN- $\gamma$ present in $\Delta$ dbIGATA mice at $14 \mathrm{dpi}$ compared to their WT counterparts (Figure 3-8D). This suggests that eosinophils may contribute to the cytokine response following $C$. burnetii infection.

When evaluating the primary antibody response to vaccination, we found that at the time of challenge there was no significant difference in total serum IgM (Figure 3-8E). Interestingly, we did find a significant decrease in total serum IgG in $\Delta$ dbIGATA mice compared to their WT counterparts. Two weeks post-challenge we detected elevated serum IgM (Figure $3-8 \mathrm{~F}$ ) in $\triangle \mathrm{dbIGATA}$ mice and no difference in serum $\lg G$, similar to the phenotype seen in $\triangle \mathrm{dbIGATA}$ mice challenged $7 \mathrm{dpv}$. In line with previous results, we also found $\triangle \mathrm{dbIGATA}$ mice to have significantly reduced IgG1 and IgG2a subclass antibodies (Figure 3-8F). Taken together, these data indicate a role for eosinophils in isotype switching for the generation of Coxiella-specific antibodies. 

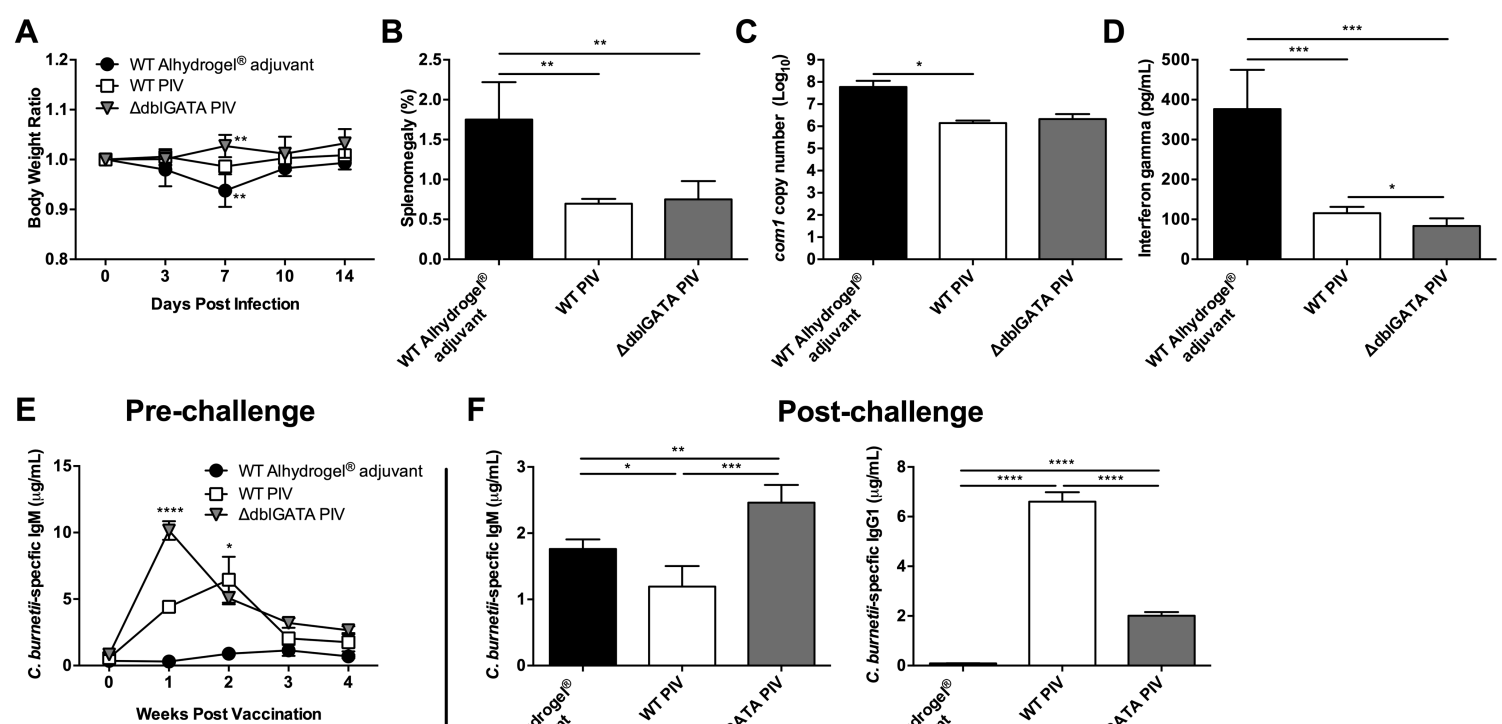

$\mathbf{F}$

Post-challenge
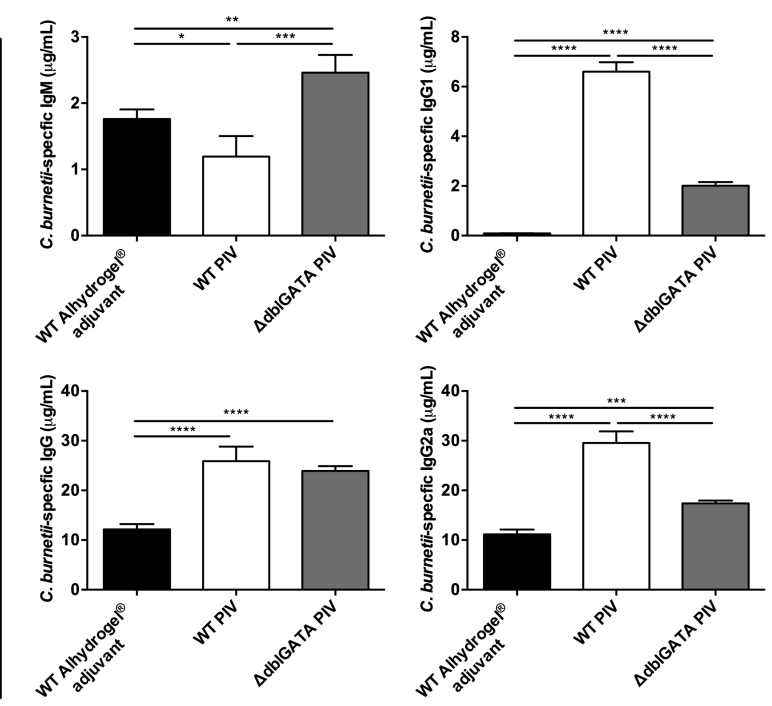

Figure 3-8. Eosinophils contribute to antibody isotype switching in late PIV responses

WT and $\triangle \mathrm{db}$ IGATA mice were vaccinated s.c. with $10 \mu \mathrm{g}$ of PIV 4 weeks prior to i.p. challenge with $1 \times 10^{7}$ C. burnetii NMI bacteria. (A) Relative body weight was measured throughout infection. Splenomegaly (B) and bacterial burden in the spleen (C) were evaluated at 14 dpi to assess disease severity. (D) Serum IFN- $\gamma$ levels were quantified at 14 dpi by cytokine-specific ELISA. $C$. burnetii NMI-specific serum IgM and IgG levels were measured weekly until $28 \mathrm{dpv}(\mathrm{E})$ as well as $14 \mathrm{dpi}(\mathrm{F})$. Results are expressed as percent splenomegaly [(spleen weight/body weight)x100]. Bacterial burden was determined by $\mathrm{qPCR}$ and is expressed as $\log _{10} C$. burnetii com1 gene copy numbers. Each experimental group includes 4 mice, with error bars representing the standard deviations from the means. ${ }^{*}, p<0.05 ;{ }^{* *}, p<0.01 ;{ }^{* * *}, p<0.001 ;{ }^{* * \star}, p<0.0001$, as determined by two-way ANOVA with Dunnett's multiple-comparison test (for panels $A$ and D) or by one-way ANOVA with Tukey's multiple-comparison test (for panels B, C, and E). 


\section{Discussion}

In this study, we sought to test the protective efficacies of PIV and PIIV generated by axenic culture methods. Reactogenicity has been attributed, in part, to residual egg proteins from the vaccine manufacturing process (197). By utilizing the axenic culture system, we removed the possibility of contamination by host-derived components. In line with previous results utilizing formalin-inactivated whole-cell vaccines derived from chicken eggs or tissue culture $(117,119,146,203)$, axenically-generated PIV was able to prevent clinical disease in BALB/c mice. There was a significant reduction in splenomegaly and an approximately 2-log decrease in $C$. burnetii genomic equivalents. Further investigation is required to determine if there is a decrease in adverse reactions with the use of this vaccine. Baeten et al. recently published a standardized guinea pig model for $Q$ fever vaccine reactogenicity testing that could prove useful for these studies (204).

A recent improvement on ACCM-2, ACCM-D, allows for the generation of 5-10 fold more bacteria with increased viability (205). Future studies examining the immunogenicity and protectivity of vaccines propagated in this defined axenic medium would be interesting considering these changes in $C$. burnetii replication dynamics. Furthermore, the higher bacterial yield would improve the feasibility of this propagation method for future vaccine manufacturing.

While whole-cell inactivated $C$. burnetii vaccines have been demonstrated to be highly efficacious without adjuvant $(35,135,136,206,207)$, these studies were carried out in healthy adults. It has recently been reported that children's risk of 
exposure to $C$. burnetii in South West Queensland is similar to the high risk of abattoir workers (208); however, $\mathrm{Q}-\mathrm{VAX}{ }^{\circledR}$ is not currently recommended for children (209). Vaccines that are successfully tested in healthy adults often fail in hypo- or non-responders such as young children and the elderly (210). Immunopotentiation in these risk groups should be the focus when designing a new generation $Q$ fever vaccine, and adjuvantation is one approach to this aim. Additionally, the use of adjuvant for immunopotentiation may allow for a reduction in the amount of antigen required to generate protection. This "dose sparing" approach may, in turn, reduce adverse reactions as they appear to occur in a dosedependent manner (207).

Previous reports in human cohorts suggested that it took at least 2-3 weeks for protection to develop $(35,135,136)$. However, this was based on the incidence of clinical $Q$ fever cases in vaccinated groups. Considering an incubation period of 23 weeks, it is likely that these patients were exposed before vaccination. Using our previously established mouse splenomegaly model, we determined that axenically-generated PIV can generate a protective response as early as $7 \mathrm{dpv}$. At this time point, there was a significant reduction in splenomegaly as well as a roughly $4 \log$ decrease in $C$. burnetii genomic equivalents. This finding has important implications in the context of $C$. burnetii's use as a biological weapon. A vaccine that generates protection in a short time frame is not only a great prophylactic candidate but also a potential therapeutic. 
The early time point at which PIV was able to significantly reduce splenomegaly suggested that innate-driven mechanisms were involved in early vaccine protection. Indeed, we found significantly more SSC ${ }^{\text {high }}$ granulocytes in the spleens of PIV-vaccinated mice at all time points. There also appeared to be an increase in the number of lymphocytes $14 \mathrm{dpv}$. Considering the kinetics of the anti-Coxiella antibody response, it is likely that this initial increase is due to the expansion of $B$ cells in response to antigen stimulus. We see peak serum IgM titers at 1 week post-vaccination and begin to detect measurable changes to serum IgG as early as 2 weeks post-vaccination.

Further characterization of the SSChigh granulocyte population indicated that they were $\mathrm{CD} 11 \mathrm{~b}^{+} \mathrm{CD} 125^{+}$Siglec- $\mathrm{F}^{+}$eosinophils. Eosinophils are typically associated with clearance of helminth infections and allergic diseases $(211,212)$; however, they are also capable of bridging innate and adaptive immunity (202). These granulocytic cells contain preformed cytokines, chemokines and growth factors that can be released quickly following activation (213). These mediators can activate neutrophils, immature dendritic cells, B cells and T cells (214).

Major basic protein (MBP) has been shown to increase superoxide production in neutrophils (215). Elliot et al. have shown that neutrophils are important for the secondary response against $C$. burnetii (216). It is possible that eosinophil activation aids in the bactericidal activity of neutrophils, which could be important for protection. Alternatively, eosinophil-derived neurotoxin (EDN) released from eosinophils stimulated with CpG DNA can elicit the maturation of immature 
dendritic cells $(217,218)$. Dendritic cells are known to play a pivotal role in generating protective adaptive responses following vaccination against many intracellular pathogens, including C. burnetii (125). Therefore, it is possible that eosinophils are the first cells to respond in a cascade involving other innate populations which then prime naïve B and T cells. However, eosinophils can also express MHC-II and co-stimulatory molecules and serve as antigen-presenting cells (APCs) to directly activate B and T cells $(214,219)$. In fact, previous studies have found alum adjuvant induces eosinophil recruitment and activation of $B$ cells to produce antigen-specific IgM (220).

Evaluating the role of eosinophils in host defense against $C$. burnetii using a more clinically-relevant aerosol model of infection is an exciting area for future research. While eosinophils are canonically known for their role in the clearance of parasitic infections, these cells are frequently involved in allergic airway inflammation (211, 212). With this in mind, it is possible that eosinophils could be involved in $C$. burnetii lung pathology. Previous reports have shown that MBP can dampen superoxide anion production by guinea pig alveolar macrophages (221). Considering C. burnetii possesses superoxide dismutase abilities (222-225), which promote its survival within the PV, it is conceivable that this function of MBP would aid in bacterial survival. However, recent reports have shown eosinophils also aid in the clearance of prominent pulmonary pathogens like respiratory syncytial virus (226231) and M. tuberculosis $(232,233)$. Indeed, Shi et al. (234) have previously demonstrated that airway eosinophils, or those isolated from the peritoneal cavity 
of hypereosinophilic IL-5 transgenic mice, migrate to paratracheal lymph nodes following instillation into the trachea and act as APCs to stimulate antigen-specific CD4 ${ }^{+} \mathrm{T}$ cell responses.

To understand the mechanism of eosinophil accumulation, we studied potential upstream signaling events that would elicit eosinophil accumulation in PIVvaccinated spleens. CD4 ${ }^{+} \mathrm{T}$ cells can recruit eosinophils through their secretion of chemoattractants like IL-5 and eotaxin- 1 . Therefore, to test whether CD4+ $\mathrm{T}$ cells were required for eosinophil accumulation, we vaccinated CD4 KO mice and compared their splenic eosinophil numbers to WT mice. Interestingly, we found that CD4 KO mice had a defect in tissue eosinophilia compared to WT mice, which suggests a $\mathrm{CD}^{+} \mathrm{T}$ cell-dependent role for this phenomenon. Additionally, we found that PIV-vaccinated CD4 KO mice had a defect in IFN- $\gamma$ production upon ex vivo restimulation with $C$. burnetii NMI. This could be due to the absence of $\mathrm{CD}^{+}$ $\mathrm{T}$ cells; however, it is also possible that the lack of significant eosinophil accumulation contributes to this phenotype. While eosinophils are best known for their release of type 2 cytokines (IL-4, IL-5, IL-13), it has become recently appreciated that these cells can also secrete many pro-inflammatory cytokines including tumor necrosis factor-alpha (TNF- $\alpha$ ) and IFN- $\gamma$ (235). Indeed, PIVvaccinated eosinophil-deficient $\Delta$ dbIGATA mice had significantly lower serum IFN$\gamma$ than PIV-vaccinated WT mice $14 \mathrm{dpi}$, which suggests that eosinophils contribute to the IFN- $\gamma$ response following $C$. burnetii infection. Further studies are required 
to determine which cell population(s) serve as early IFN- $\gamma$ producers in our vaccine model.

To evaluate the contribution of eosinophils to PIV protection, we vaccinated eosinophil-deficient $\triangle \mathrm{dbIGATA}$ mice and challenged them either 7 or $28 \mathrm{dpv}$. $\triangle \mathrm{dbIGATA}$ mice have a deletion in the GATA1 promoter, which is important for eosinophil development (178). We found $\triangle$ dbIGATA mice had a defect in protection when challenged $7 \mathrm{dpv}$. This suggests that eosinophils may play a partial role in the early protective response against $C$. burnetii. Considering the changes in splenomegaly and bacterial burden were relatively small, albeit statistically significant, it is possible that eosinophils do not make a biologically significant contribution to vaccine immunity. However, it would be interesting to evaluate vaccine protection using a hypereosinophilic IL-5 transgenic mouse model. Eosinophil recruitment to the spleen, while not sufficient for vaccine protection, could enhance the overall vaccine response. In this case, adjuvant selection targeting eosinophils would be an interesting approach to consider.

Two weeks post-challenge, $\triangle$ dbIGATA mice vaccinated 7 days prior displayed elevated serum IgM with no difference in total IgG compared to WT. However, there was a significant decrease in both $\lg G 1$ and $\lg G 2 a$ subclass antibodies. While there was no difference in the total $\lg G$ concentration, the fact that we saw decreases in certain IgG subclasses indicates that the absence of eosinophils likely affects the ratio of each subclass (lgG1, $\lg G 2 a, \lg G 2 b, \lg G 3)$ which shapes the overall antibody response. Indeed, eosinophils have been shown to promote 
$\mathrm{B}$ cell responsiveness to both $\mathrm{T}$-dependent and $\mathrm{T}$-independent antigens in the periphery (236). Furthermore, previous work by Chu et al. has shown that eosinophils are required for the maintenance of long-lived plasma cells in the bone marrow $(237,238) . \triangle$ dbIGATA mice have normal plasmablast migration to the bone marrow, however, they fail to develop into mature plasma cells (239). In line with these results, we found $\Delta \mathrm{dbIGATA}$ mice challenged $28 \mathrm{dpv}$ to have elevated serum IgM with decreased IgG1 and IgG2a subclass antibodies. This data indicates that eosinophils contribute to isotype switching and help shape the humoral response.

Cumulatively, this data has led us to propose a working model (Figure 3-9) to describe the role of eosinophils in PIV-mediated protection. In our proposed model, APCs, such as dendritic cells (DCs) (240), process and present vaccine antigens to naïve CD4+ $T$ cells. These activated helper cells secrete IL-5, which is a potent chemoattractant and activator of eosinophils. Eosinophils then accumulate in the spleens of vaccinated animals, where they are poised to respond to subsequent infection. Upon secondary challenge, eosinophils can release pre-formed IFN- $\gamma$ from their granules. IFN- $\gamma$ aids in bacterial clearance through activation of macrophages and enhancement of their microbicidal activity (241), promotion of IgG2a secretion from B cells $(242,243)$, and expansion of Th1 ${ }^{+} \mathrm{CD} 4^{+} \mathrm{T}$ cells $(244)$. While it still remains to be demonstrated that eosinophils secrete IFN- $\gamma$ in response to $C$. burnetii, it is known that IFN- $\gamma$ promotes the previously mentioned host defense mechanisms against $C$. burnetii $(117,171,173,174,201,245-247)$. 
In summary, this study provides novel information regarding the utility of axenic culture for the generation of highly efficacious vaccines against $C$. burnetii. Additionally, our results shed light on a previously uncharacterized mechanism of vaccine protection involving $\mathrm{CD} 11 \mathrm{~b}+\mathrm{CD} 125^{+}$Siglec $-\mathrm{F}^{+}$eosinophils. Further understanding of the mechanisms of vaccine protection will guide future rational vaccine design and adjuvant selection. 


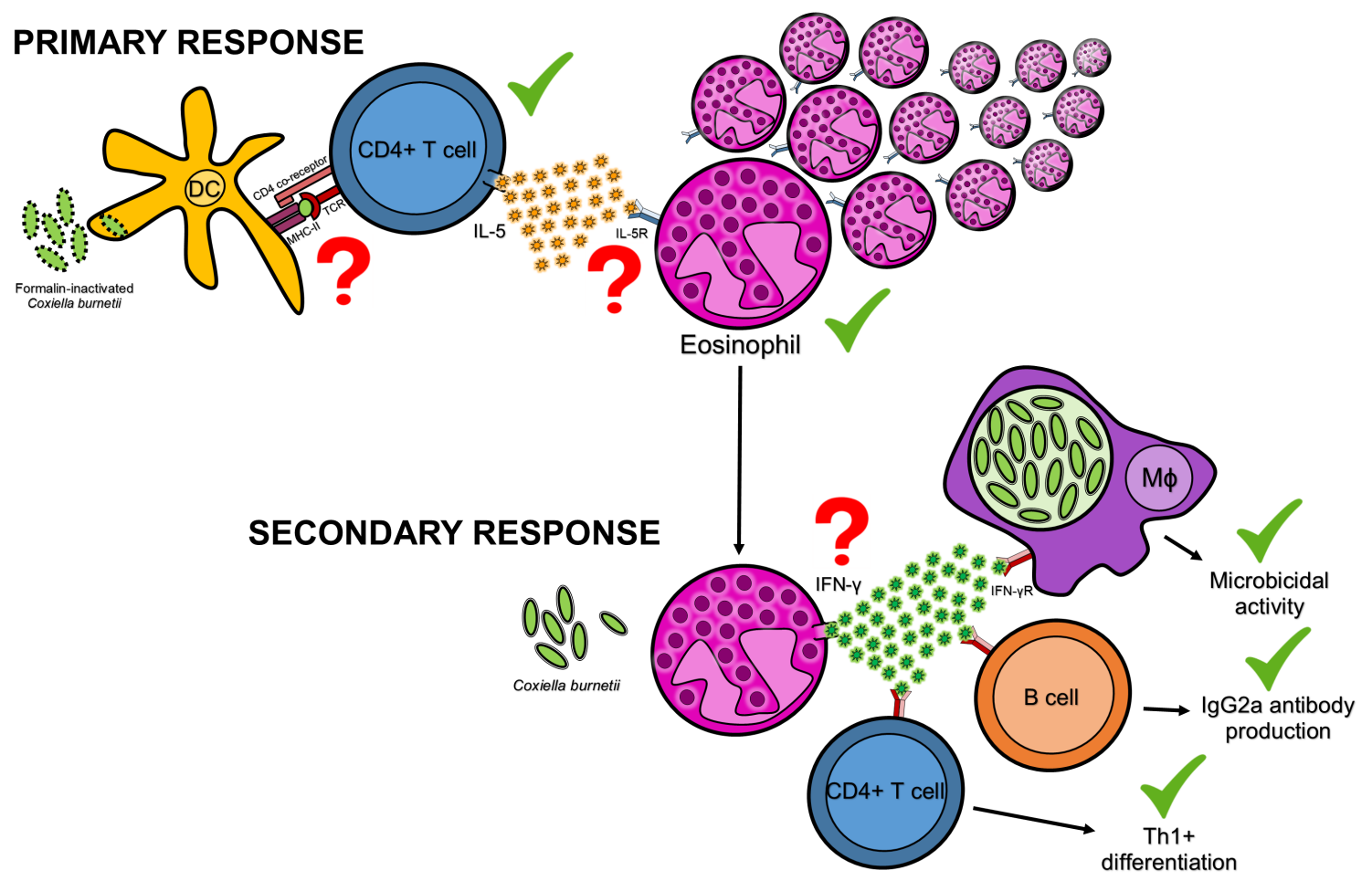

Figure 3-9. A working model for eosinophils in PIV-mediated protection

During the primary response, vaccine antigens are taken up by APCs, such as dendritic cells, processed, and presented to naïve $\mathrm{CD}^{+}{ }^{+} \mathrm{T}$ cells. These naïve cells become activated $\mathrm{T}$ helper cells and can secrete IL-5, a potent chemoattractant and activator of eosinophils. Eosinophils then accumulate in the spleens of vaccinated animals, where they are poised to respond to subsequent infection. Upon secondary challenge, eosinophils can release preformed IFN- $\gamma$ from their granules. IFN- $\gamma$ contributes to host immunity against $C$. burnetiit through activation of macrophages and enhancement of their microbicidal activity, promotion of IgG2a secretion from B cells, and expansion of Th1+ helper cells. Steps denoted with a green check mark $(\checkmark)$ have been demonstrated either experimentally in this chapter or previously in the literature. Red question marks (?) denote steps which require further experimentation. 


\section{CHAPTER 4: MHC-II-RESTRICTED, CD4+ T CELL- DEPENDENT AND -INDEPENDENT MECHANISMS ARE REQUIRED FOR VACCINE-INDUCED PROTECTIVE IMMUNITY AGAINST COXIELLA BURNETII}

\section{Abstract}

To understand the role of major histocompatibility complex class I (MHC-I) and class II (MHC-II) in vaccine-mediated protection against $C$. burnetii, we evaluated the protective efficacy of a formalin-inactivated $C$. burnetii Nine Mile phase I vaccine (PIV) in beta-2-microglobulin-deficient (B2m KO) and MHC-II-deficient (MHC-II KO) mice. Vaccination reduced disease severity in wild-type (WT) and B2m KO mice, but failed to reduce bacterial burden in MHC-II KO mice. This suggests that the MHC-II antigen presentation pathway is required for PIVmediated protection against $C$. burnetii infection. MHC-I and MHC-II affect antibody isotype switching, as both PIV-vaccinated B2m KO and MHC-II KO mice produced less Coxiella-specific IgG than PIV-vaccinated WT mice. Interestingly, MHC-II and CD4 deficiency were not equivalent in terms of splenomegaly and bacterial clearance. This demonstrates a partial role for $\mathrm{CD}^{+}{ }^{+} \mathrm{T}$ cells while revealing MHC-II-restricted, CD4-independent mechanisms. Adoptive transfer of CD4 ${ }^{+} \mathrm{T}$ cells from PIV-vaccinated WT mice to naïve CD4-deficient (CD4 KO) mice demonstrated that antigen-experienced $\mathrm{CD} 4^{+} \mathrm{T}$ cells are sufficient to generate protection. Conversely, transfer of naïve CD4+ $\mathrm{T}$ cells to PIV-vaccinated CD4 KO mice exacerbates disease. Using Tbet-deficient mice (Tbet KO), we showed a 
partial role for Th1 subset CD4+ $\mathrm{T}$ cells in vaccine protection. Furthermore, Th1independent roles for Tbet were suggested by significant differences in disease between PIV-vaccinated Tbet KO and CD4 KO mice. Interferon-gamma (IFN- $\gamma$ ) was shown to contribute to the host inflammatory response, but not bacterial clearance. Collectively, these findings suggest that vaccine-induced protective immunity against a murine model of experimental $Q$ fever requires MHC-IIrestricted, $\mathrm{CD}^{+} \mathrm{T}$ cell-dependent and -independent mechanisms that can be exploited for a new-generation human $Q$ fever vaccine.

\section{Introduction}

A protective immune response involves cooperation between the innate and adaptive arms of the immune system. Following infection or vaccination, professional APCs such as DCs, macrophages, and B cells recognize components of the foreign material through PRRs on their cell surface. Following the recognition of PAMPs, APCs internalize and process these complex antigens into peptides which can then be loaded into MHC complexes for transport back to the cell surface. Peptide-MHC complexes interact with naïve $T$ cells through the $T$ cell receptor and, with proper co-stimulation and cytokine signals, lead to their activation. Canonically, MHC-I complexes signal to $\mathrm{CD} 8^{+}$cytotoxic $\mathrm{T}$ cells, while MHC-II signals $\mathrm{CD}^{+} \mathrm{T}$ helper cells. Effector $\mathrm{T}$ cells secrete cytokines and/or release cytotoxic molecules for pathogen clearance, while a subset of activated T cells will become memory cells. Concomitantly, B cells recognize antigens through the $\mathrm{B}$ cell receptor and secrete neutralizing IgM antibody. Humoral and CMI 
intersect through CD40-CD40L signaling, in which activated CD4+ T helper cells aid B cells in antibody isotype switching. As with T cells, subsets of B cells will remain as memory cells and long-lived plasma cells. Memory $\mathrm{T}$ and B cells are poised to rapidly respond upon subsequent exposures, and their generation is the goal of vaccination. Identifying the signals necessary to generate sufficient memory (i.e. correlates of immunity) is required for a rational approach to vaccine development.

It has previously been demonstrated that both humoral and cell-mediated immunity contribute to host defense against $C$. burnetii $(98,117,119,127,164,167,168$, $170-175,201,248)$. In a murine i.p. infection model, B cells appear to contribute to the host inflammatory response, while $\mathrm{T}$ cells and IFN- $\gamma$ are important for bacterial clearance (201). However, only the adoptive transfer of immune T cells, not immune B cells, from PIV-vaccinated BALB/c mice to SCID mice reduces disease severity following i.p. challenge (117). These data suggest an important role for T cells in both the primary and secondary host response against $C$. burnetii. In a primary pulmonary infection model, either $\mathrm{CD} 4^{+}$or $\mathrm{CD} 8^{+} \mathrm{T}$ cells are sufficient to control infection, with $\mathrm{CD}^{+} \mathrm{T}$ cells better controlling inflammation (172). This is in line with our recently published data demonstrating that both MHC-I and MHCII are important for primary host defense, with MHC-I playing a more significant role (175). Similarly, either $\mathrm{CD}^{+}$or $\mathrm{CD}^{+} \mathrm{T}$ cells are sufficient to generate protection following vaccination with PIV (119), however, the role of MHC-I and MHC-II in vaccine-induced protective immunity is unknown. It remains unclear how 
$\mathrm{CD}^{+}$and $\mathrm{CD}^{+} \mathrm{T}$ cells contribute to vaccine protection, and which specific $\mathrm{T}$ cell subsets respond to vaccination is not clear.

Here we investigate the role of $\mathrm{MHC}-\mathrm{I}$ and $\mathrm{MHC}-\mathrm{II}$ in vaccine protection against $C$. burnetii and show that MHC-II is important for PIV-mediated protection. The contribution of $\mathrm{MHC}-\mathrm{II}$ to vaccine-induced protective immunity is only partially dependent on $\mathrm{CD}^{+}{ }^{+} \mathrm{T}$ cells, as PIV-vaccinated MHC-II KO mice have significantly worse disease than PIV-vaccinated CD4 $\mathrm{KO}$ mice. $\mathrm{CD}^{+} \mathrm{T}$ cells are, however, sufficient for protection when they come from an antigen-experienced donor. This is demonstrated by a significant reduction in splenomegaly following the adoptive transfer of PIV-vaccinated CD4+ $\mathrm{T}$ cells to naïve CD4 KO mice. Furthermore, we demonstrate a role for Tbet in PIV protection that is partially dependent on Th1 subset $C D 4^{+} T$ cells. When evaluating the contribution of IFN- $\gamma$ we found that, while IFN- $\gamma$ does seem to affect inflammation, it does not appear to play a major role in bacterial clearance following secondary challenge. These findings provide novel information about the role of MHC-II, Tbet, $\mathrm{CD}^{+}{ }^{+} \mathrm{T}$ cells, and IFN- $\gamma$ in vaccineinduced protective immunity against a murine model of experimental $Q$ fever. Furthermore, this study highlights key differences in the host response following primary and secondary C. burnetii challenge which can inform future $Q$ fever vaccine development. 


\section{Results}

\section{MHC-II is important for PIV-mediated protection against $C$. burnetii}

We have previously demonstrated that both MHC-I and MHC-II play important roles in host defense against primary $C$. burnetii infection, with MHC-I being more critical (175). To determine the role of these complexes in vaccine-mediated protection, we vaccinated MHC-I-deficient (B2m KO) and MHC-II KO mice s.c. with $10 \mu \mathrm{g}$ PIV with Alhydroge $\left.\right|^{\circledR}$ adjuvant followed by i.p. challenge with $1 \times 10^{7} \mathrm{C}$. burnetii NMI $28 \mathrm{dpv}$. An aluminum hydroxide adjuvant was chosen for these studies based on their widely accepted use in commercially-available human vaccines (198). Body weight loss, splenomegaly, and splenic bacterial burden were evaluated to assess the protective efficacy of PIV. PIV-vaccinated WT C57BL/6 mice were protected from body weight loss compared to WT adjuvant control mice, which had a significant drop in body weight $7 \mathrm{dpi}$ (Figure 4-1A). This correlated with a significant reduction in splenomegaly (Figure 4-1D) and reduced splenic bacterial loads (Figure 4-1E) in PIV-vaccinated WT mice compared to adjuvant controls. Vaccination did not change the course of body weight loss in B2m KO mice, which displayed a transient loss in body weight beginning $7 \mathrm{dpi}$ (Figure 4-1B). However, vaccination did protect B2m KO mice as demonstrated by significant reductions in splenomegaly (Figure 4-1D) and bacterial burden (Fig 41E). The most significant drop in body weight occurred in MHC-II KO mice, which lost approximately $15 \%$ of their initial body weight by 7 dpi (Figure $4-1 C$ ). Vaccination of MHC-II KO mice partially abrogated this weight loss, as the PIV- 
vaccinated cohort had significantly higher body weights 7,10 , and $14 \mathrm{dpi}$. However, PIV-vaccinated MHC-II KO mice still lost $\sim 10 \%$ of their initial body weight by 7 dpi. In line with these results, PIV-vaccinated MHC-II KO mice only had a small, albeit statistically significant, reduction in splenomegaly (Figure 4-1D) and no change in splenic bacterial loads (Figure 4-1E). Overall, these data indicate that the MHC-II antigen presentation pathway is important for PIV-mediated protection against $C$. burnetii.

Next, we evaluated the development of specific antibody titers following vaccination and challenge. PIV-vaccinated WT mice produced significant levels of Coxiella-specific IgM, peaking $7 \mathrm{dpv}$, compared to WT adjuvant-treated controls (Figure 4-2A). Vaccination also elicited specific IgM in B2m KO (Figure 4-2B) and MHC-II KO mice (Figure 4-2C), which peaked $7 \mathrm{dpv}$. However, the overall IgM concentrations were markedly reduced compared to PIV-vaccinated WT mice. IgM levels were similar between vaccinated groups at the time of challenge. Significant C. burnetii NMI-specific IgG production began $14 \mathrm{dpv}$ in PIV-vaccinated WT mice and continued to rise until the time of challenge (Figure 4-2D). In contrast, the concentration of specific IgG produced by PIV-vaccinated B2m KO (Figure 4-2E) and MHC-II KO mice (Figure 4-2F) was markedly reduced. When evaluated 14 dpi, PIV-vaccinated WT mice had significantly elevated IgM (Figure 4-2G) and IgG (Figure 4-2H) compared to adjuvant-treated controls. Adjuvant-treated and PIVvaccinated B2m KO mice produced similar levels of specific IgM (Figure 4-2G), indicating that vaccination had no effect on IgM production in the absence of MHC- 
I. In contrast, PIV-vaccinated MHC-II KO mice had significantly higher levels of IgM compared to adjuvant-treated controls (Figure 4-2G). Similar to PIV-vaccinated WT mice, PIV-vaccinated B2m KO and MHC-II KO mice produced significantly higher specific IgG than adjuvant treated controls following challenge (Figure 4$2 \mathrm{H})$. However, the levels of specific IgG were markedly reduced in the absence of MHC-I or MHC-II. Collectively, these data suggest a role for both MHC-I and MHCII in antibody isotype switching.

\section{MHC-II-dependent vaccine protection partially depends on $\mathrm{CD}^{+} \mathrm{T}$ cells}

$\mathrm{CD}^{+} \mathrm{T}$ cells are the primary target of $\mathrm{MHC}$-II-mediated antigen presentation. Therefore, to determine the contribution of $\mathrm{CD}^{+}{ }^{+} \mathrm{T}$ cells to PIV-mediated protection, we vaccinated and challenged CD4 KO and MHC-II KO mice as described previously. Body weight loss, splenomegaly, and splenic bacterial burden were evaluated to assess vaccine protection. CD4 KO and MHC-II KO mice had similar body weight loss, which was significantly worse than PIV-vaccinated WT mice 7 and 10 dpi (Figure 4-3A). CD4 KO mice also had significant body weight loss compared to PIV-vaccinated WT mice $14 \mathrm{dpi}$. At this time point, CD4 KO and MHC-II KO mice both had significantly worse splenomegaly compared to PIVvaccinated WT mice (Figure 4-3B). Interestingly, the degree of splenomegaly in MHC-II KO mice was significantly worse than in CD4 KO mice. In line with these results, MHC-II KO mice had a higher splenic bacterial burden than PIV-vaccinated WT or CD4 KO mice (Figure 4-3C). Bacterial loads were similar between PIVvaccinated WT and CD4 KO mice, which also had similar degrees of spleen 
A

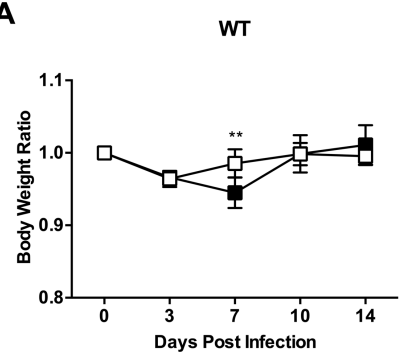

D

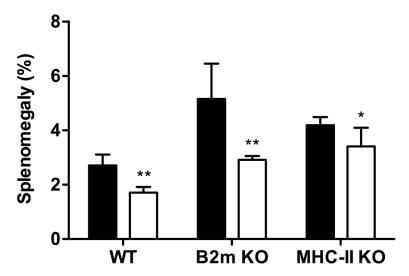

B

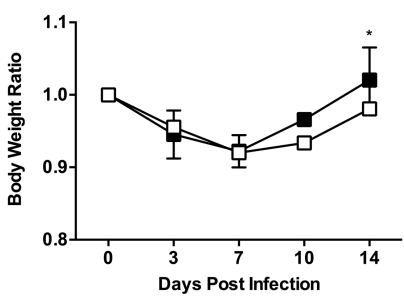

E

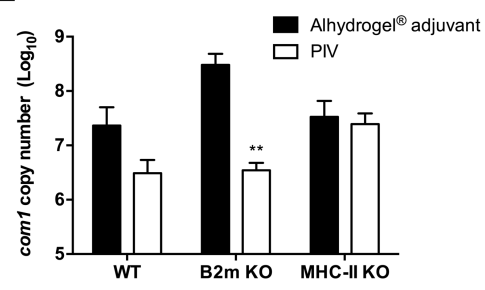

Figure 4-1. MHC-II is important for PIV-mediated protection against $C$. burnetii

B2m-deficient (B2m KO), MHC-II-deficient (MHC-II KO), and WT C57BL/6 mice were vaccinated S.c. with $10 \mu \mathrm{g}$ PIV and challenged i.p. with $1 \times 10^{7}$ C. burnetii NMI bacteria $28 \mathrm{dpv}$. Mice receiving Alhydroge ${ }^{\circledR}$ adjuvant alone served as unvaccinated controls. (A to C) Relative body weight was calculated throughout infection to monitor disease progression. Splenomegaly (D) and splenic bacterial burden (E) were evaluated 14 dpi to compare protection. Results are expressed as percent splenomegaly [(spleen weight/body weight)x100]. Bacterial burden was determined by qPCR and is expressed as $\log _{10} C$. burnetii com1 gene copy numbers. Each experimental group includes 5 mice with error bars representing the standard deviations from the means. ${ }^{*}, p<0.05$; ${ }^{\star \star}, \mathrm{p}<0.01 ;{ }^{* \star \star}, \mathrm{p}<0.001 ;{ }^{* \star \star *}, \mathrm{p}<0.0001$ as determined by two-way ANOVA with Sidak's multiplecomparison test (for panels $\mathrm{A}$ to $\mathrm{C}$ ) or by t-test (for panels $\mathrm{D}$ and $\mathrm{E}$ ). 

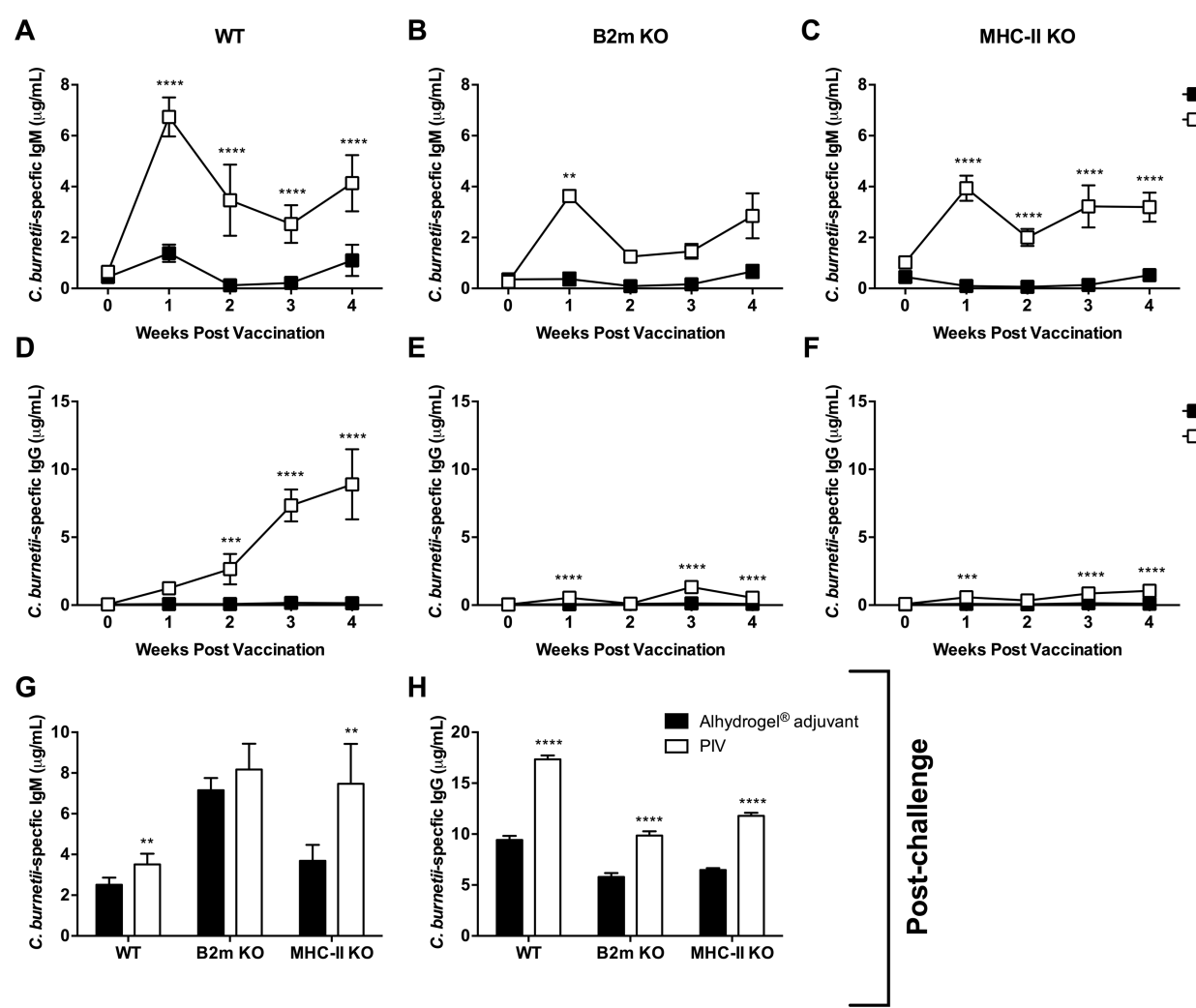

Figure 4-2. MHC-I and MHC-II are involved in antibody isotype switching

B2m KO, MHC-II KO, and WT C57BL/6 mice were vaccinated S.c. with $10 \mu \mathrm{g}$ PIV and challenged i.p. with $1 \times 10^{7}$ C. burnetii NMI bacteria $28 \mathrm{dpv}$. Mice receiving Alhydrogel ${ }^{\circledR}$ adjuvant alone served as unvaccinated controls. C. burnetii NMI-specific serum IgM ( $A$ to $C$ ) and IgG ( $D$ to $F$ ) were evaluated weekly following vaccination. Specific $\lg M(G)$ and $\lg G(H)$ were also evaluated $14 \mathrm{dpi}$. Each experimental group includes 5 mice with error bars representing the standard deviations from the means. ${ }^{* \star}, p<0.01 ;{ }^{* \star *}, p<0.001 ;{ }^{* * *}, p<0.0001$ as determined by two-way ANOVA with Sidak's multiple-comparison test (for panels $A$ to $F$ ) or by t-test (for panels $G$ and $H$ ). 
pathology (Figures 4-3D and 4-3E). In contrast, MHC-II KO mice had significantly worse spleen pathology than both PIV-vaccinated WT and CD4 KO mice. These results suggest that $\mathrm{CD} 4^{+} \mathrm{T}$ cells may only play a partial role in MHC-II-dependent vaccine protection.

When evaluating the Coxiella-specific antibody response following vaccination, CD4 KO and MHC-II KO mice had significantly less IgM and undetectable levels of IgG compared to PIV-vaccinated WT mice (Figure 4-4A). At 14 dpi, both CD4 $\mathrm{KO}$ and MHC-II KO mice had significantly higher IgM than PIV-vaccinated WT mice as well as significantly less IgG (Figure 4-4B). This suggests $C D 4^{+} T$ cells are important for isotype switching and the generation of a protective antibody response against $C$. burnetii. However, the fact that MHC-II KO mice had significantly lower IgG titers and more severe splenomegaly than CD4 KO mice suggests additional CD4-independent mechanisms may be involved. Collectively, these data indicate that $\mathrm{CD} 4+\mathrm{T}$ cells partially contribute to the MHC-II-dependent protection generated by PIV.

\section{$\mathrm{CD4}^{+} \mathrm{T}$ cells from PIV-vaccinated mice are sufficient to generate protection}

To further examine the role of $\mathrm{CD}^{+}{ }^{+} \mathrm{T}$ cells in PIV-mediated protection, we obtained purified CD4 ${ }^{+} \mathrm{T}$ cells from naïve WT mice and WT mice which had been vaccinated with PIV as previously described. Approximately $5 \times 10^{6}$ purified CD4+ $\mathrm{T}$ cells were adoptively transferred to naïve CD4 KO mice via i.p. injection 24 hours before injection with $C$. burnetii. Body weight loss, splenomegaly, and splenic bacterial burden were evaluated to assess the protective efficacy of CD4 ${ }^{+} \mathrm{T}$ cell 

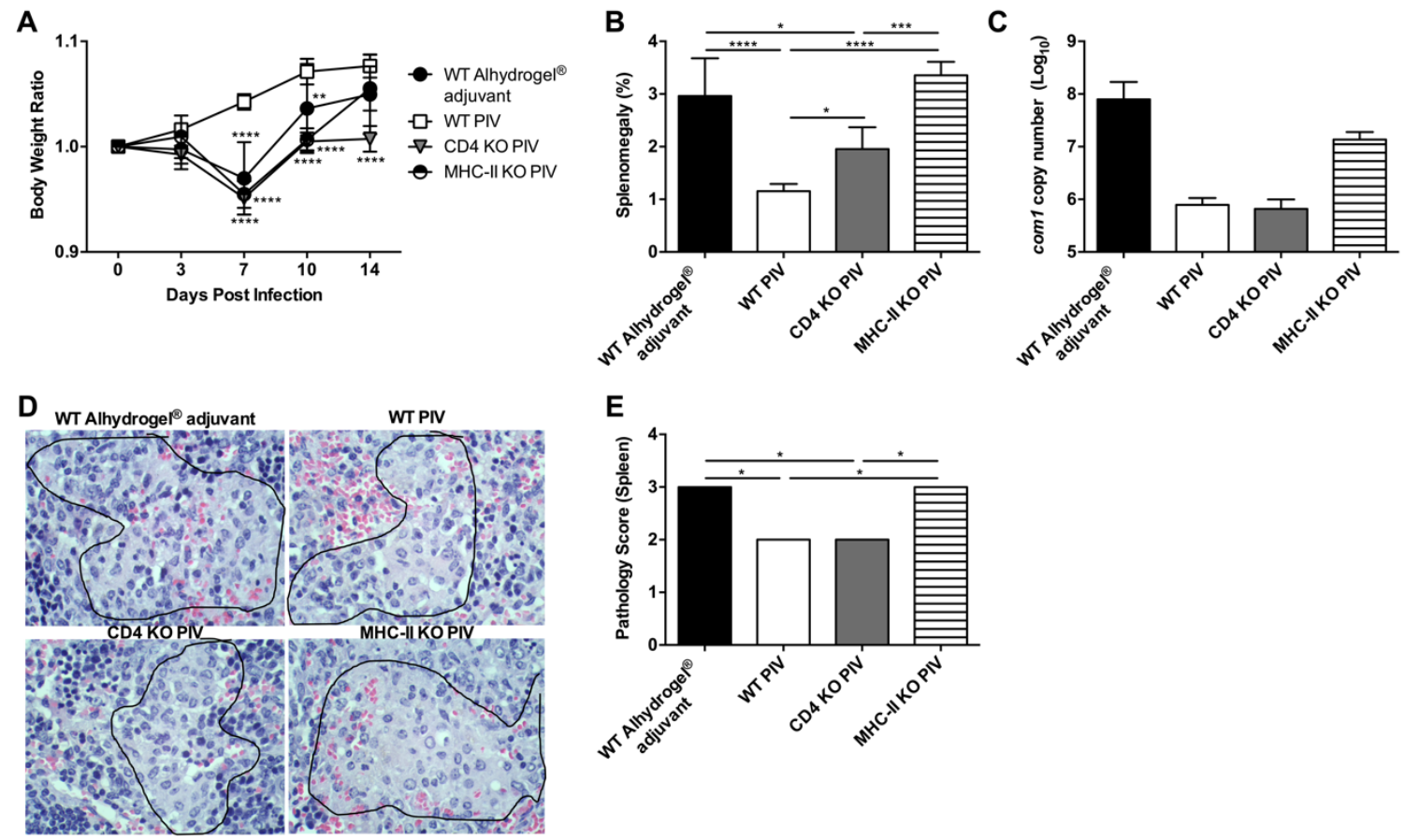

Figure 4-3. MHC-II-dependent vaccine protection is partially dependent on CD4+ $\mathrm{T}$ cells

CD4-deficient (CD4 KO), MHC-II KO, and WT C57BL/6 mice were vaccinated s.c. with $10 \mu \mathrm{g}$ PIV and challenged i.p. with $1 \times 10^{7} \mathrm{C}$. burnetii NMI bacteria $28 \mathrm{dpv}$. Mice receiving Alhydrogel ${ }^{\circledR}$ adjuvant alone served as unvaccinated controls. (A) Relative body weight was determined throughout infection to assess morbidity. Splenomegaly (B) and splenic bacterial burden (C) were assessed 14 dpi to compare protection. (D and E) Spleen sections from WT, CD4 KO, and MHCII KO mice were evaluated 14 dpi for histiocytic inflammation in red pulp based on the following scale: 0 , no accumulations of macrophages; 1 , small accumulations of macrophages; 2 , small to moderate accumulations of macrophages; and 3, moderate to large accumulations of macrophages. Differences in spleen volume between groups were largely the result of extramedullary hematopoiesis. Results are expressed as percent splenomegaly [(spleen weight/body weight)x100]. Bacterial burden was determined by $\mathrm{qPCR}$ and is expressed as $\log _{10}$ C. burnetii com1 gene copy numbers. Each experimental group includes 5 mice with error bars representing the standard deviations from the means. ${ }^{*}, p<0.05 ;{ }^{* \star}, p<0.01 ;{ }^{\star \star \star}, p<0.001 ;{ }^{* \star \star \star}$, $\mathrm{p}<0.0001$ as determined by two-way ANOVA with Dunnett's multiple-comparison test (for panel A), by one-way ANOVA with Tukey's multiple-comparison test (for panels B and C), or by KruskalWallis with Dunn's multiple-comparison test (for panel E). 
A

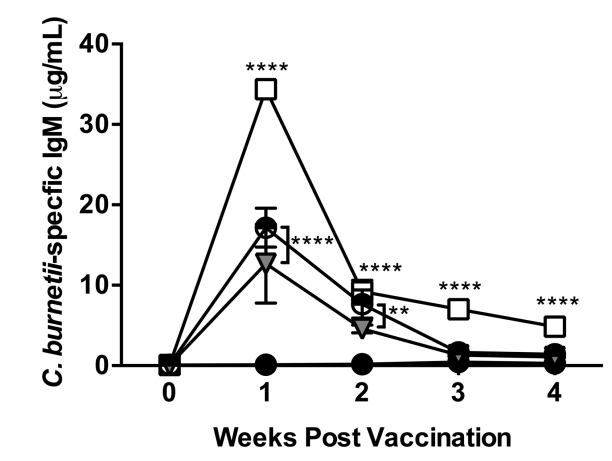

Pre-challenge

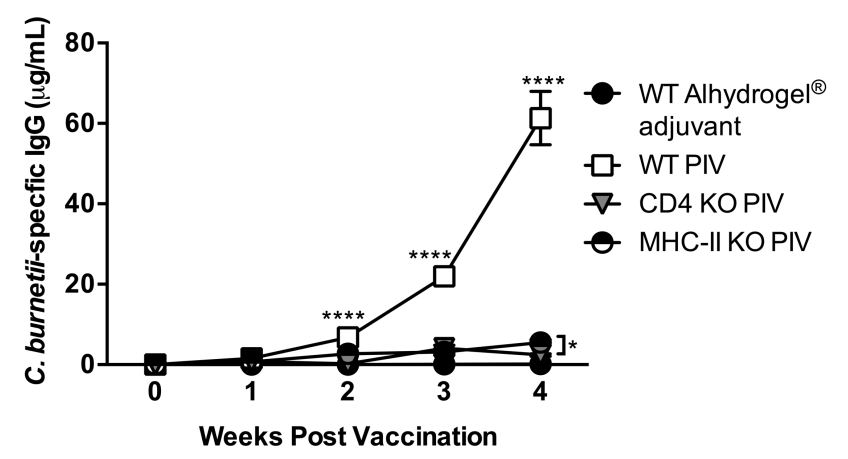

B

Post-challenge

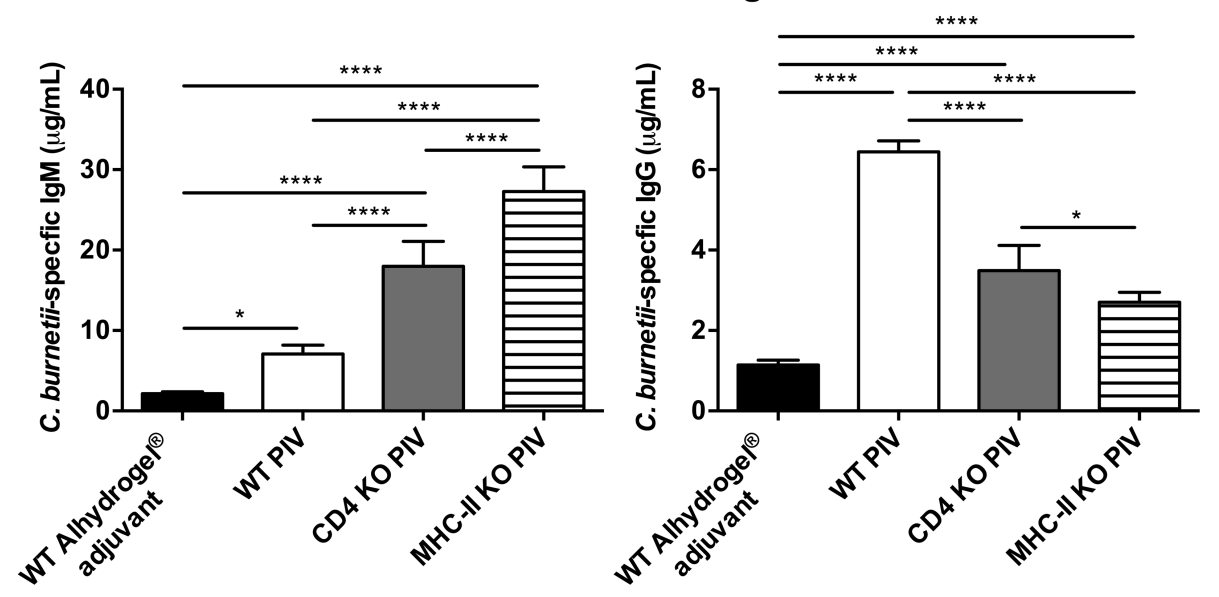

Figure 4-4. CD4+ $T$ cells are important for antibody isotype switching

WT, CD4 KO, and MHC-II KO mice were vaccinated and challenged as previously described. Mice receiving Alhydroge ${ }^{\circledR}$ adjuvant alone served as unvaccinated controls. C. burnetii NMIspecific serum IgM and IgG were evaluated (A) weekly until $28 \mathrm{dpv}$ and (B) $14 \mathrm{dpi}$. Each experimental group includes 5 mice with error bars representing the standard deviations from the means. ${ }^{*}, p<0.05 ;{ }^{* \star}, p<0.01 ;{ }^{* * * *}, p<0.0001$ as determined by two-way ANOVA with Tukey's multiple-comparison test (for panel A) or by one-way ANOVA with Tukey's multiple-comparison test (for panel B). 
transfer. PIV-vaccinated CD4 KO mice, as well as all CD4 KO recipient mice, had significant body weight loss throughout infection compared to PIV-vaccinated WT mice (Figure 4-5A). However, by $14 \mathrm{dpi}$, the recipient mice which received PIVvaccinated $C D 4^{+} T$ cells had increased body weight compared to those recipients who received naïve $\mathrm{CD}^{+}{ }^{+} \mathrm{T}$ cells. Furthermore, $\mathrm{CD} 4 \mathrm{KO}$ recipient mice receiving PIV-vaccinated $\mathrm{CD}^{+} \mathrm{T}$ cells had a significant reduction in splenomegaly which was similar to PIV-vaccinated WT and CD4 KO mice (Figure 4-5B). Similarly, CD4 KO recipient mice receiving PIV-vaccinated $\mathrm{CD}^{+} \mathrm{T}$ cells had splenic bacterial loads comparable to PIV-vaccinated WT and CD4 KO mice (Figure 4-5C). This is in contrast to $\mathrm{CD} 4 \mathrm{KO}$ recipient mice receiving naïve $\mathrm{CD} 4^{+} \mathrm{T}$ cells, which had similar splenomegaly and bacterial burden to WT adjuvant control mice. In summary, these data indicate that CD4+ $\mathrm{T}$ cells from PIV-vaccinated WT mice are sufficient to generate protection and reduce clinical disease in naïve CD4 KO mice. To determine if this phenotype was the result of direct antigen stimulation of CD4+ T cells, we next performed an adoptive transfer of naïve or PIV-vaccinated CD4+ T cells to PIV-vaccinated CD4 KO mice. We again saw significant body weight loss in PIV-vaccinated CD4 KO mice as well as all CD4 KO recipient mice compared to PIV-vaccinated WT mice (Figure 4-6A). The most significant body weight loss was seen in PIV-vaccinated CD4 $\mathrm{KO}$ mice which received naïve $\mathrm{CD} 4^{+} \mathrm{T}$ cells. In line with these results, PIV-vaccinated CD4 KO mice which received naïve CD4+ T cells had significant splenomegaly (Figure 4-6B), equivalent to that of WT adjuvant control mice, as well as higher splenic bacterial loads (Figure 4-6C). This 


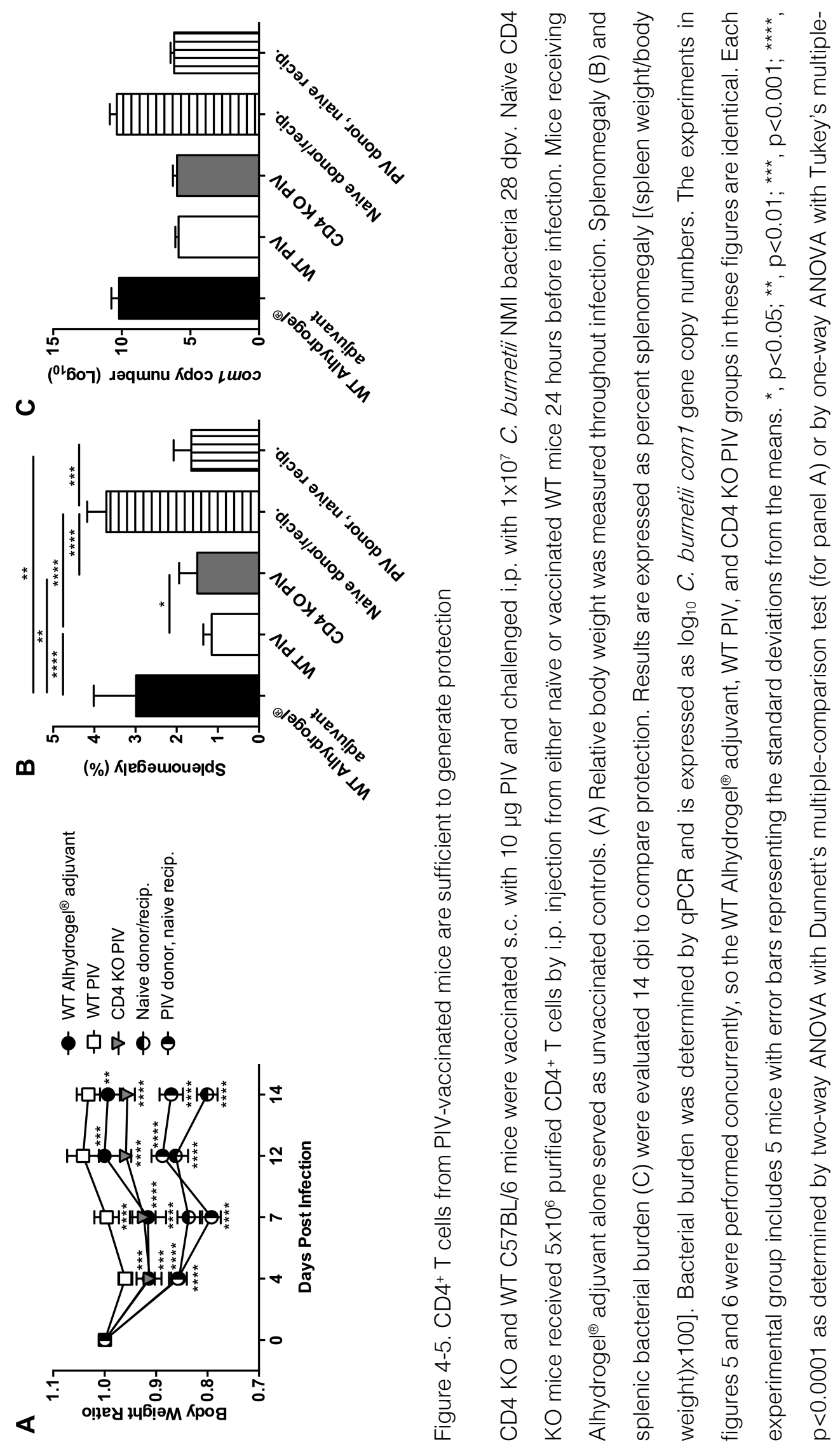



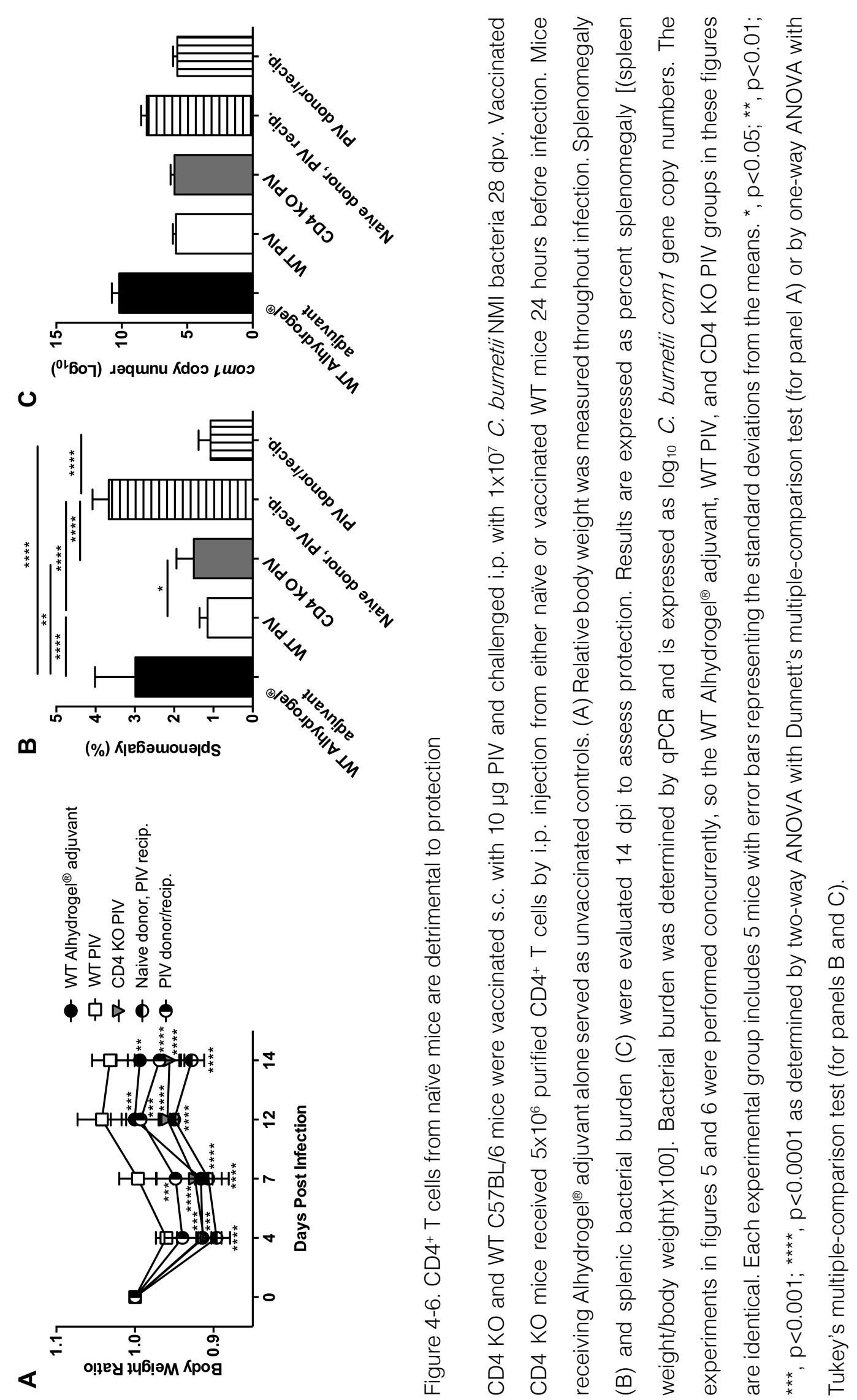
suggests that naïve $C D 4^{+} T$ cells may play a detrimental role when introduced into a PIV-vaccinated CD4-deficient host. In contrast, PIV-vaccinated CD4 KO recipients which received $\mathrm{CD}^{+}{ }^{+} \mathrm{T}$ cells from PIV-vaccinated WT donors had splenomegaly and bacterial burden comparable to PIV-vaccinated WT mice. Overall, these data suggest that $\mathrm{CD} 4^{+} \mathrm{T}$ cells from PIV-vaccinated WT mice are sufficient to generate protection, while those from naïve donors may exacerbate disease.

\section{Tbet plays a role in PIV protection that partially depends on Th1 CD4+ $\mathrm{T}$ cells} Naïve CD4+ $T$ cells can differentiate into different $T$ helper subsets (e.g. Th1, Th2, Th17, T follicular helper, T regulatory, etc.) based on transcriptional regulation and the local cytokine environment (249). To determine which $\mathrm{CD}^{+}{ }^{+} \mathrm{T}$ helper subsets are involved in vaccine protection, we vaccinated and challenged transcription factor deficient mice as previously described. Body weight loss, splenomegaly, and splenic bacterial burden were evaluated to assess vaccine protection. Tbet KO mice, which are deficient in the Th1 subset and display markedly reduced IFN- $\gamma$ (183), showed significant body weight loss compared to PIV-vaccinated WT mice 3, 7, and $10 \mathrm{dpi}$ (Figure 4-7A). No significant body weight loss occurred in Stat6 $\mathrm{KO}$ mice, which are non-responsive to IL-4/IL-13 signaling and lack the Th2 subset (184). Th17-deficient Roryt KO mice (185) did not lose significant body weight until $14 \mathrm{dpi}$. At this time point, Tbet KO mice had significantly worse splenomegaly than PIV-vaccinated WT mice (Figure 4-7B). In contrast, both Stat6 KO and Roryt KO mice had similar splenomegaly to PIV-vaccinated WT mice. Considering Tbet KO 


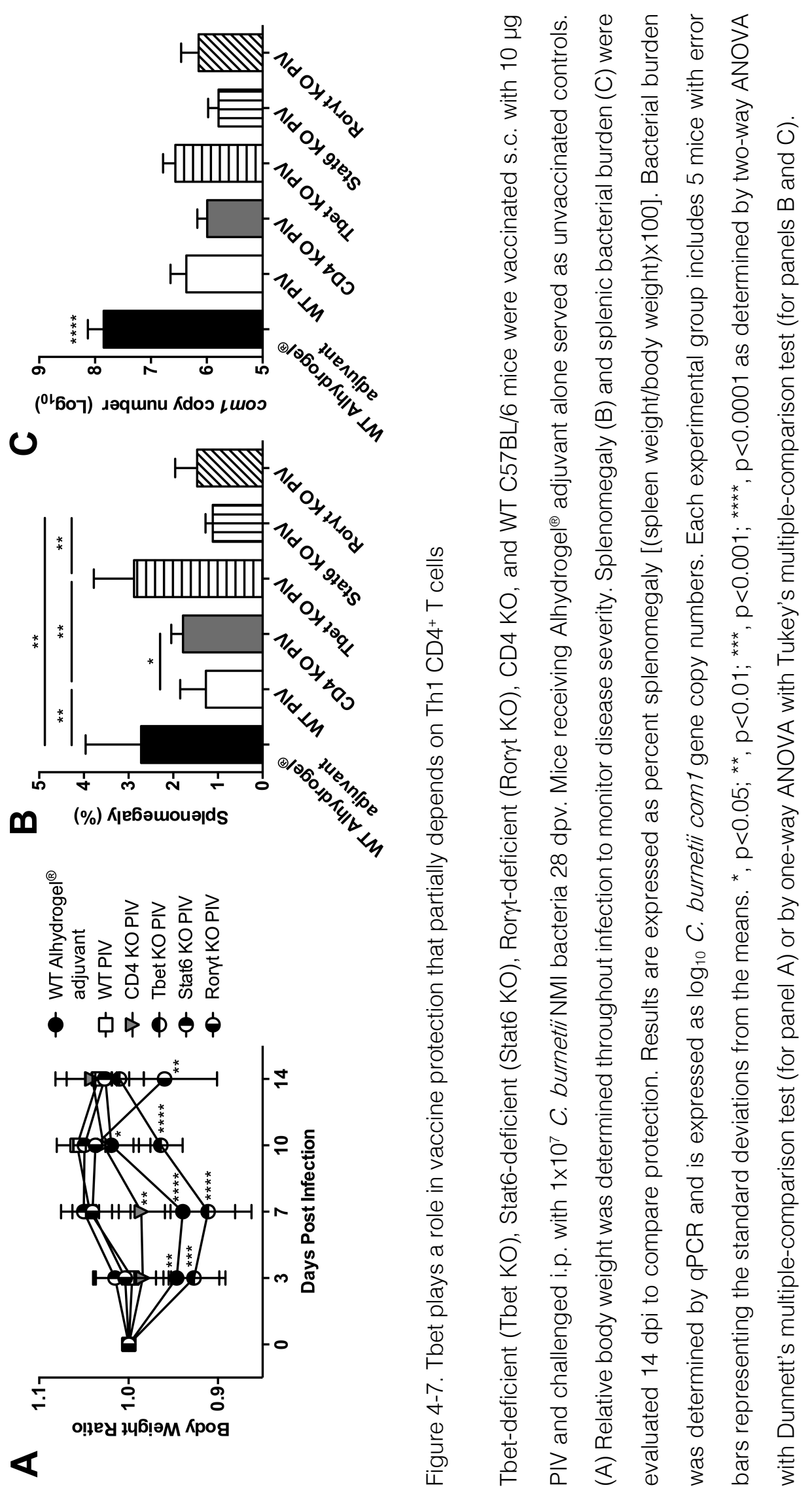


mice had worse splenomegaly than CD4 KO mice, there may be a role for Tbet in vaccine protection that is independent of its role in Th1 differentiation. In terms of splenic bacterial burden, all deficient strains had a statistically significant reduction compared to WT adjuvant controls and were similar to PIV-vaccinated WT mice (Figure 4-7C). This suggests that Th1 cells serve a role in inflammation modulation, though they may not contribute to bacterial clearance. Taken together, these results indicate that Tbet plays a role in vaccine protection which partially depends on Th1 CD4+ $\mathrm{T}$ cells.

\section{IFN- $\gamma$ modulates inflammation but may not be involved in bacterial clearance}

IFN- $\gamma$, in conjunction with Tbet, influences the differentiation of naïve T cells into the Th1 subset. Furthermore, IFN- $\gamma$ has been previously reported to play an important role in the clearance of $C$. burnetii following primary challenge (201). To evaluate the role of IFN- $\gamma$ in PIV-mediated protection, we vaccinated and challenged IFN- $\gamma$-deficient (IFN- $\gamma \mathrm{KO}$ ) mice and measured body weight loss, splenomegaly, and splenic bacterial burden. IFN- $\gamma \mathrm{KO}$ mice had significant body weight loss compared to PIV-vaccinated WT mice beginning 7 dpi (Figure 4-8A). Furthermore, IFN- $\gamma \mathrm{KO}$ mice had significantly worse splenomegaly than PIVvaccinated WT mice 14 dpi (Figure 4-8B). Interestingly, we saw no significant difference in splenic bacterial burden between PIV-vaccinated WT and IFN- $\gamma$ KO mice (Figure 4-8C). Collectively, these results suggest that IFN- $\gamma$ may not be required for bacterial clearance during secondary challenge and may only serve to modulate the host inflammatory response. 


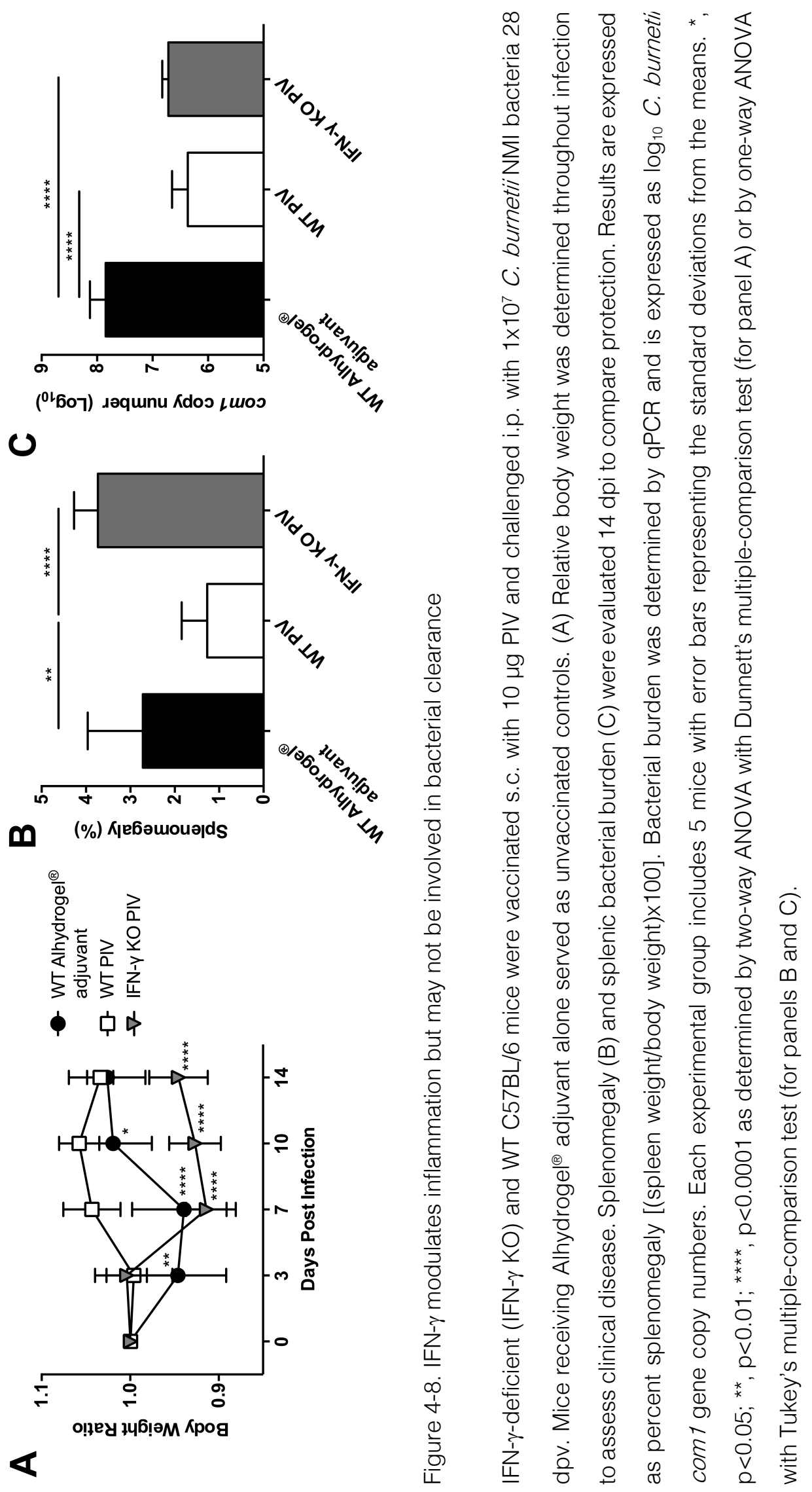




\section{Discussion}

Whole-cell inactivated $C$. burnetii vaccines, including $Q-V A X^{\circledR}$, are highly efficacious in healthy volunteers without adjuvant $(35,135,136,206,207)$. Still, vaccines which are successfully tested in healthy adults are often nonimmunogenic in young children and the elderly (210). Indeed, it is reported that children's risk of exposure to C. burnetii in South West Queensland is similar to the high risk of abattoir workers (208) and yet $\mathrm{Q}-\mathrm{VAX}{ }^{\circledR}$ is not currently recommended for children (209). As such, immunopotentiation in these risk groups should be the focus of a new generation $Q$ fever vaccine. Adjuvantation is one approach to this aim. Furthermore, the addition of adjuvant to enhance immunogenicity may allow for reduction in the amount of $C$. burnetii antigen required to generate protection. This "dose sparing" approach may, in turn, reduce reactogenicity since it appears to occur in a dose-dependent manner (207).

MHC molecules guide T cell development and shape adaptive immunity. In the thymus, MHC-I and MHC-II select for $\mathrm{CD}^{+}$and $\mathrm{CD} 4^{+} \mathrm{T}$ cells, respectively. As such, mice deficient in beta-2-microglobulin (B2m), a component of MHC-I, do not express MHC-I on their cell surface and are virtually devoid of CD8 ${ }^{+} \mathrm{T}$ cells (180). Similarly, MHC-II KO mice lack CD4+ $T$ cells (181). Using these deficient mouse strains, we have previously shown that both MHC-I and MHC-II are required for host defense against primary $C$. burnetii infection, with MHC-I deficiency having a greater impact on disease severity (175). In this study, we sought to determine the role of MHC-I and MHC-II in PIV-mediated immunity and found that, in contrast to 
primary infection, MHC-II appears to play an important role during secondary responses. PIV-vaccinated MHC-II KO mice had significant body weight loss, a mild reduction in splenomegaly, and no change in com1 gene copy numbers compared to adjuvant controls. MHC-II is important for vaccine immunity against other intracellular pathogens, including herpes simplex virus $(250,251)$, influenza virus (252), and M. bovis Bacillus Calmette-Guerin (253). In these models, the importance of MHC-II is associated with $\mathrm{CD} 4^{+} \mathrm{T}$ cell help for the generation of protective antibody. When we evaluated the Coxiella-specific antibody response following vaccination, we found that PIV-vaccinated B2m KO and MHC-II KO mice had early defects in IgM which were restored to WT levels at the time of challenge. Additionally, PIV-vaccinated $\mathrm{B} 2 \mathrm{~m} \mathrm{KO}$ and MHC-II KO mice produced markedly reduced IgG over the course of vaccination. The lack of Coxiella-specific IgG in PIV-vaccinated B2m KO mice was unexpected, however, there are at least two possible explanations for this phenomenon. B2m is not only involved in the assembly of MHC-I but it is also a component of the neonatal Fc receptor (FcRn) (254). As such, B2m KO mice do not express FcRn. FcRn has been shown to protect IgG from catabolism, thus the lack of IgG could be due to rapid turnover in this mouse strain (255-257). Indeed, it has been previously demonstrated that B2m $\mathrm{KO}$ mice produce significantly less virus-specific $\lg \mathrm{G}$ in response to vaccinia virus infection than their WT counterparts (258). Alternatively, a follicular $\mathrm{CD}^{+} \mathrm{T}$ cell subset has recently been described which promotes antibody isotype switching through the secretion of IL-21 (259). The latter may be more likely considering both 
PIV-vaccinated $\mathrm{B} 2 \mathrm{~m} \mathrm{KO}$ and $\mathrm{MHC}-\mathrm{II} \mathrm{KO}$ mice produced IgG following challenge, albeit less than WT mice. Further investigation is required to understand the role MHC-I and MHC-II play in generating Coxiella-specific protective antibodies.

Following the observation that MHC-II KO mice were more severely affected than B2m KO mice, we wanted to evaluate the role of $\mathrm{CD}^{+} \mathrm{T}$ cells in PIV protection. $\mathrm{CD}^{+} \mathrm{T}$ cells receive signals from professional APCs through $\mathrm{T}$ cell receptor recognition of peptide-loaded MHC-II. Interestingly, we found that disease burden in CD4 KO mice was not equivalent to MHC-II KO mice. PIV-vaccinated CD4 KO mice had significantly elevated splenomegaly compared to PIV-vaccinated WT mice; however, their splenomegaly and splenic bacterial burden were still significantly less than MHC-II KO mice. This suggests that CD4-independent mechanisms may exist which contribute to vaccine protection in the absence of CD4. It has previously been reported that $\mathrm{MHC}$-II-restricted $\mathrm{CD} 4{ }^{-} \mathrm{CD} 8^{+}$and $\mathrm{CD} 4^{-}$ CD8- $a \beta^{+} T$ cells expand to fill the T cell compartment in the absence of CD4 (179, 260-264). These noncanonical T cell subsets are capable of protecting mice from bacterial and viral infections $(260,261,263)$. Indeed, protection from lethal Leishmania major in CD4 KO mice was found to depend on IFN- $\gamma$-secreting, MHCII-restricted CD4-CD8- double negative $a \beta^{+} \mathrm{T}$ cells (260). Furthermore, similar to classical CD4 ${ }^{+} \mathrm{T}$ cells, these cells are capable of B cell helper functions. Zheng et al. (265) found that germinal center formation, Ig somatic hypermutation, affinity maturation, and B cell memory generation were all able to progress normally in CD4 KO mice, albeit at a lower level. When we examined the Coxiella-specific 
antibody response in CD4 KO mice following vaccination, we found that they did not make significant specific IgG compared to PIV-vaccinated WT mice. However, they did produce significantly higher levels of specific IgG compared to MHC-II KO mice and WT adjuvant controls following challenge. This suggests that the contribution of one of these noncanonical MHC-II-restricted T cell subsets is possible, however, they are not as efficient as $\mathrm{CD}^{+}{ }^{+} \mathrm{T}$ cells considering CD4 KO mice had significantly less IgG than WT mice. $y \delta^{+} T$ cells are another population that could be mediating protection in the absence of CD4. Schneider et al. (266) reported elevated CD45RO+HLA-DR+ $\gamma^{+} \delta^{+} T$ cells in patients with acute $Q$ fever compared to healthy controls. These $y \delta^{+} T$ cells were more activated than $a \beta^{+} T$ cells, which suggests an important role for them in host defense against $C$. burnetii. The role these unique $T$ cell populations play in vaccine-mediated immunity is an interesting area for future research.

To further investigate the function of $\mathrm{CD}^{+} \mathrm{T}$ cells in PIV-mediated protection, we next performed adoptive transfer experiments to $\mathrm{CD} 4 \mathrm{KO}$ mice. $\mathrm{CD} 4^{+} \mathrm{T}$ cells from PIV-vaccinated WT donor mice resulted in similar splenomegaly and splenic bacterial burden to PIV-vaccinated WT mice when administered to naïve CD4 KO mice, which suggests that antigen-experienced $C D 4^{+} \mathrm{T}$ cells are sufficient to generate protection. We use the term antigen-experienced to describe $\mathrm{T}$ cells derived from an animal that has previously been vaccinated, realizing that the isolated cells are a mixed population of naïve and antigen-specific memory $T$ cells. The fact that PIV is a whole-cell vaccine with multiple antigens precludes the use 
of $\mathrm{MHC}$ tetramers for isolation of antigen-experienced $\mathrm{CD} 4^{+} \mathrm{T}$ cells. While $\mathrm{CD} 4^{+} \mathrm{T}$ cells from PIV-vaccinated WT donor mice are sufficient to protect naïve CD4 KO recipients, naïve $\mathrm{CD} 4^{+} \mathrm{T}$ cells appear to exacerbate disease in PIV-vaccinated CD4 KO recipients. This suggests that vaccination reprograms $\mathrm{CD}^{+} \mathrm{T}$ cells. Indeed, Zhang et al. (117) reported decreased serum IL-12p40, TNF-a, IFN- $\gamma$, IL17 and G-CSF in vaccinated mice following i.p. challenge with $C$. burnetii NMI compared to non-protective PIIV and adjuvant controls. With this in mind, we hypothesize that the transfer of naïve CD4+ T cells to PIV-vaccinated CD4 KO mice results in elevated proinflammatory cytokines. It would be interesting to evaluate the differences in cytokine responses between PIV-vaccinated CD4 KO mice and PIV-vaccinated CD4 KO mice which received naïve CD4+ $\mathrm{T}$ cells following ex vivo restimulation with $C$. burnetii NMI.

Naïve CD4+ $T$ cells can differentiate into different effector subsets (Th1, Th2, Th17, $\mathrm{T}$ regulatory, $\mathrm{T}$ follicular helper) following activation. Th1 subset $\mathrm{CD} 4^{+} \mathrm{T}$ cells are a proinflammatory population that is transcriptionally regulated by Tbet (267). Their development is further driven by IL-12 and IFN- $\gamma$. Th2 cells are canonically involved in antibody-mediated immunity, and their development is guided by GATA-3 and IL-4/Stat6 $(184,268,269)$. Th17 subset CD4 ${ }^{+} T$ cells develop through signals from ROR $\gamma t$ and IL-23 (270). To understand the role of these subsets in PIV-mediated immunity, we utilized Tbet-deficient, Stat6-deficient, and ROR $\gamma t$-deficient mice. Stat6 and ROR $\gamma$ t KO mice had similar splenomegaly and bacterial burden to PIVvaccinated WT mice, which suggests that Th2 and Th17 subset $C D 4^{+} \mathrm{T}$ cells do 
not contribute significantly to protection. Conversely, Tbet KO mice had significantly worse splenomegaly than PIV-vaccinated WT mice. This suggests that Th1 cells are important for PIV-mediated protection. Interestingly, Tbet KO mice had worse disease than CD4 KO mice, indicating that there are likely Th1independent roles for Tbet in vaccine immunity. Indeed, Tbet is known to be involved in the development and effector function of many other cell subsets including dendritic cells, B cells, CD8 ${ }^{+} \mathrm{T}$ cells, and NK cells. DCs have been shown to require Tbet for the production of IFN- $\gamma$ and activation of antigen-specific $\mathrm{T}$ cell responses (271). As such, the phenotype seen in Tbet KO mice may be the result of a loss of DC function. DCs are critical to vaccine protection and DCs pulsed with protein antigens from $C$. burnetii NMI have been shown to elicit a protective $\mathrm{CD}^{+}$ T cell response (125). Alternatively, Tbet is involved in isotype switching in B cells. Tbet-deficient $B$ cells fail to undergo class-switch recombination to the $\lg G 2 a$ isotype (272), especially in response to T-independent antigens like LPS (273). Antibody-mediated immunity, and more specifically lgG2a, is known to be required for PIV protection. Zhang et al. (117) reported a 4:1 ratio of IgG2a to IgG1 following vaccination with $0.2 \mu \mathrm{g}$ PIV compared to a $1: 2 \operatorname{lgG} 2 \mathrm{a} / \mathrm{lgG} 1$ ratio following vaccination with $0.2 \mu \mathrm{g}$ PIIV. Therefore, the increased disease burden observed in Tbet KO mice could be due to a lack of Coxiella-specific antibody production. Considering Tbet is involved in T-independent isotype switching, this would be a plausible CD4-independent mechanism. Indeed, it has previously been demonstrated that passive transfer of antibody produced following PIV vaccination 
of CD4 KO mice is sufficient for protection against $C$. burnetii infection (119). Otherwise, $\mathrm{CD}^{+} \mathrm{T}$ cell and $\mathrm{NK}$ cell dysfunction could also be playing a role. Effector and memory $\mathrm{CD} 8^{+} \mathrm{T}$ cell fates are regulated by Tbet and are impaired in Tbet KO mice (274-276). In the context of Tbet deficiency, the lack of a Th1 CD4+ T cell response coupled with dysfunctional $\mathrm{CD} 8^{+} \mathrm{T}$ cells likely has an additive effect on disease severity and the lack of vaccine protection. Indeed, nude mice lacking both subsets of $\mathrm{T}$ cells have worse disease than either CD4 or CD8 KO mice following vaccination and challenge with $C$. burnetii NMI (119). NK cells have not been studied in the context of $Q$ fever vaccination, however, they do not appear to play a significant role following primary challenge (201). Overall, the role of Tbet in PIV-mediated protection is likely multifaceted and will be better elucidated with adoptive transfer studies using specific Tbet deficient cell subsets.

Regardless of the cell population studied, the consensus is that defects resulting from Tbet deficiency are due to dysregulated IFN- $\gamma$. Tbet transactivates the IFN- $\gamma$ gene and has been shown to control IFN- $\gamma$ production in $\mathrm{CD}^{+} \mathrm{T}$ cells, CD8 ${ }^{+} \mathrm{T}$ cells, and NK cells (183). Furthermore, the susceptibility of Tbet KO mice to multiple other intracellular pathogens, including M. tuberculosis (277), Salmonella typhimurium (278) and L. major (183), has been attributed to insufficient IFN- $\gamma$. To determine the role of IFN- $\gamma$ in vaccine-mediated protection against $C$. burnetii, we vaccinated and challenged IFN- $\gamma \mathrm{KO}$ mice. Interestingly, while IFN- $\gamma \mathrm{KO}$ mice had more severe splenomegaly than PIV-vaccinated WT mice, there was no difference in splenic bacterial burden. This corresponds with the phenotype observed in Tbet 
$\mathrm{KO}$ mice in which there was significant splenomegaly compared to PIV-vaccinated WT mice with no difference in splenic bacterial burden. Collectively, these data suggest that Tbet and IFN- $\gamma$ modulate the host inflammatory response but do not appear to play a critical role in bacterial clearance following secondary challenge. This is in contrast to the critical role of IFN- $\gamma$ after primary challenge (201). However, we did observe differences in the kinetics of body weight loss between Tbet $\mathrm{KO}$ and IFN- $\gamma \mathrm{KO}$ mice which suggests that their roles in vaccine protection are not indistinguishable. Tbet $\mathrm{KO}$ mice displayed significant body weight loss beginning $3 \mathrm{dpi}$ that recovered to the level of PIV-vaccinated WT mice by $14 \mathrm{dpi}$. However, IFN- $\gamma$ KO mice exhibited significant body weight loss beginning $7 \mathrm{dpi}$ and failed to recover by $14 \mathrm{dpi}$. While Tbet is required for optimal IFN- $\gamma$ production, IFN$\gamma$ can be secreted in the absence of Tbet (279) and could explain this difference in body weight loss.

In summary, this study provides novel information about mechanisms of CMI in vaccine protection against a murine model of experimental $Q$ fever. We report a previously uncharacterized MHC-II-dependent mechanism of protection against $C$. burnetii that is partially dependent on Tbet, CD4+ $\mathrm{T}$ cells, and IFN- $\gamma$. Furthermore, this study highlights differences in the primary and secondary immune response which should be considered when designing future vaccines against $Q$ fever. 


\section{CHAPTER 5: DISCUSSION}

Historically, vaccines have been developed without an understanding of the mechanisms by which they protect. However, we now face diseases like HIV and tuberculosis for which this empirical approach has proven unsuccessful. Through mechanistic studies of successful vaccines, we can establish correlates of protective immunity which can guide rational vaccine design (Figure 5-1). Possessing known correlates of protection lowers the economic risk associated with vaccine development and reduces the chance of failure in clinical trials. This concept, known as systems vaccinology, inspired this work.

Q-VAX ${ }^{\circledR}$ has successfully reduced the spread of $Q$ fever among occupational risk groups in Australia since the implementation of a National Q Fever Management Program in 2001 (280). Nevertheless, it has failed to gain approval by the FDA due to adverse reactions associated with pre-existing immunity or egg allergies. Understanding which vaccine components elicit these hypersensitivities, as well as the mechanisms of protective immunity, is necessary to rationally design a safe and effective $Q$ fever vaccine. The work described here reveals innate-driven mechanisms of vaccine immunity as well as MHC-II-restricted $\mathrm{CD}^{+}{ }^{+} \mathrm{T}$ cellindependent mechanisms of $\mathrm{CMI}$ which can be integrated with the previously characterized mechanisms of vaccination discussed in Chapter 1 to inform future Q fever vaccine development. 


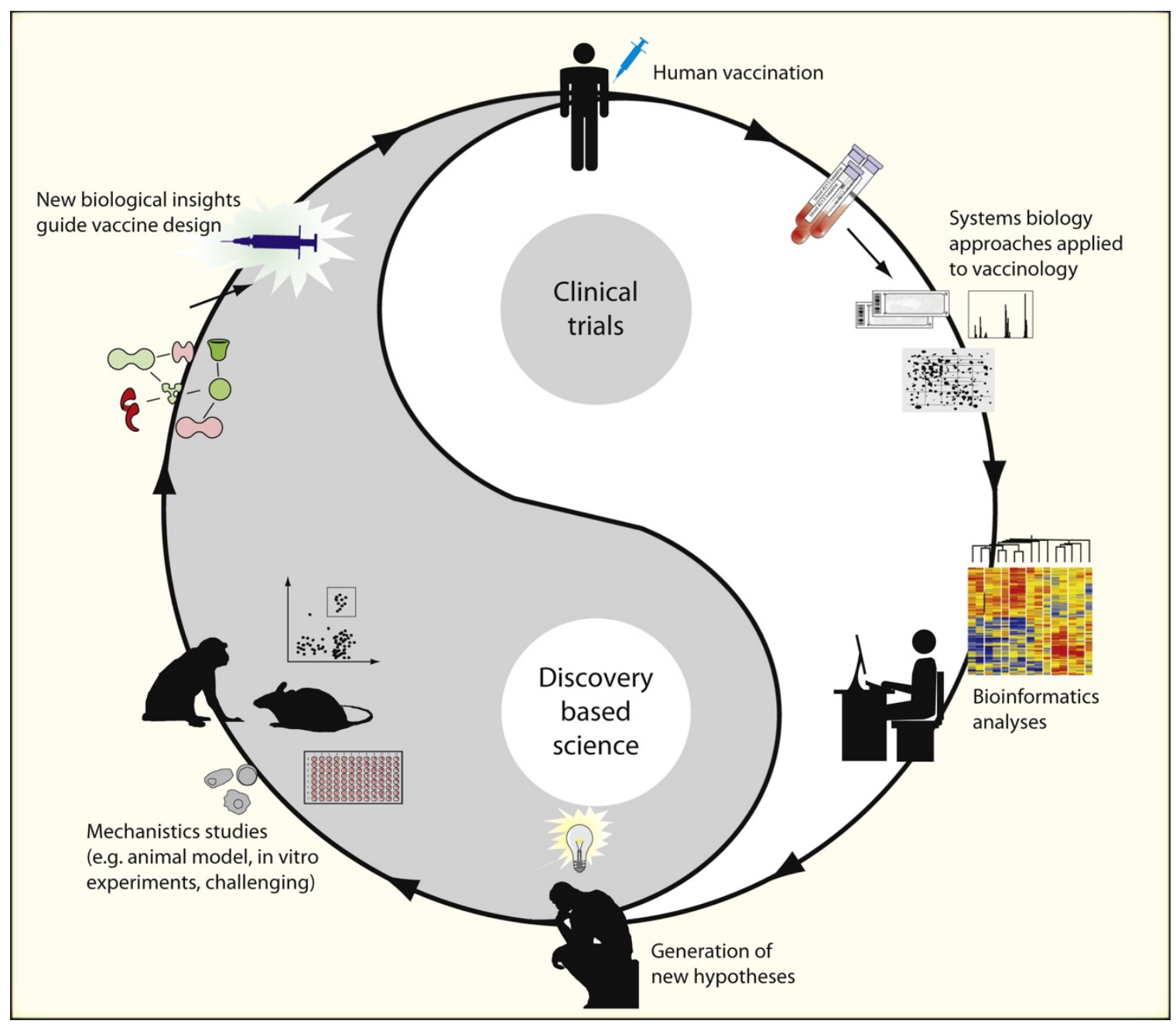

Figure 5-1. A systems vaccinology approach for rational vaccine design

The systems vaccinology approach aims to bridge the gap between clinical trials and discoverybased science. Studying our most effective human vaccines using bioinformatics analyses can generate new hypotheses about the mechanisms of protective immunity which can be tested through in vitro and in vivo mechanistic studies. This approach could provide new insights which may inform vaccine development efforts.

Adapted from Pulendran B et al. 2010. Immunity 33. 


\section{A working model of protective immunity}

Based on my data and the current literature, I've developed a working model of protective immunity against $Q$ fever which may serve as a framework for rational Q fever vaccine design (Figure 5-2). Following vaccination with PIV, B cells recognize their cognate antigen and produce Coxiella-specific IgM (119). This response occurs independently of T cells (119) and peaks at approximately $7 \mathrm{dpv}$ (Figures 3-8E, 4-2A, and 4-4A). Concurrently, professional APCs process and present vaccine antigens to $\mathrm{CD}^{+} \mathrm{T}$ cells in an MHC-II-dependent manner. DCs are hypothesized to be the main cells involved here, as vaccination with PIV results in $\mathrm{DC}$ upregulation of the chemokine receptor CCR7 and migration to secondary lymphoid organs (128). Activated CD4+ $\mathrm{T}$ cells facilitate eosinophil recruitment to the spleen at $7 \mathrm{dpv}$ (Figures 3-5 and 3-6) via an unknown mechanism. I hypothesize that Th2 cells secrete IL-5, an eosinophil chemoattractant, though this remains to be shown experimentally. Eosinophils contribute to the antibody response following vaccination, as eosinophil deficient mice produce significantly less Coxiella-specific $\lg G$ at $28 \mathrm{dpv}$ (Figure 3-8). Activated $\mathrm{CD}^{+} \mathrm{T}$ cells also facilitate the Coxiella-specific IgG response via CD40-CD40L interactions with activated B cells. This is evidenced by previous reports showing passive immunization with IgG from CD4 KO mice is not protective (119). Additionally, PIVvaccinated CD4 KO mice produce significantly less Coxiella-specific IgG than PIVvaccinated WT mice (Figure 4-4A). The predominant anti-Coxiella IgG isotype is IgG2a (117), indicating a role for IFN- $\gamma$ in the primary response. 
Protection following secondary challenge with virulent $C$. burnetii NMI is thought to occur in two phases. First, the antibody produced in response to vaccination likely neutralizes incoming $C$. burnetii bacteria. However, one would expect some percentage of the bacteria to escape antibody-mediated immunity and infect macrophages. Vaccine-induced CMI would then be necessary for the clearance of intracellular bacteria. This model is supported by the ability of immune serum to protect naïve immunocompetent mice, in which $\mathrm{CMI}$ is intact, and the inability of immune serum to protect naïve SCID mice, in which CMI is absent (117).

$\mathrm{CD}^{+} \mathrm{T}$ cells only partially contribute to vaccine-induced protective immunity against $C$. burnetii (Figure 4-3), influencing the IFN- $\gamma$ response (Figure 3-6) and the antibody response (Figure 4-4). In the absence of CD4, other MHC-II-restricted T cells expand including CD4-CD8 ${ }^{+}$and CD4-CD8 ${ }^{-} a \beta^{+} T$ cells, as well as $\gamma^{+} \delta^{+} T$ cells $(179,260-264)$. Considering the significant difference in disease severity between PIV-vaccinated CD4 KO mice and MHC-II KO mice (Figure 4-3), it is likely that one of these noncanonical MHC-II-restricted T cell populations is mediating protection in the absence of $\mathrm{CD} 4^{+} \mathrm{T}$ cells. Which specific MHC-II-restricted $\mathrm{T}$ cell population is involved remains unclear. Flow cytometric analysis of $\mathrm{T}$ cell receptor type $(a \beta, \gamma \delta)$, co-receptor expression (CD4, CD8), and activation state (CD25, CD69, KLRG1) following vaccination and challenge would be informative. Acute $Q$ fever patients have elevated $y \delta^{+} T$ cells expressing HLA-DR and CD45RO in peripheral blood, suggesting that this population may be involved in host defense against $C$. burnetii (266). 


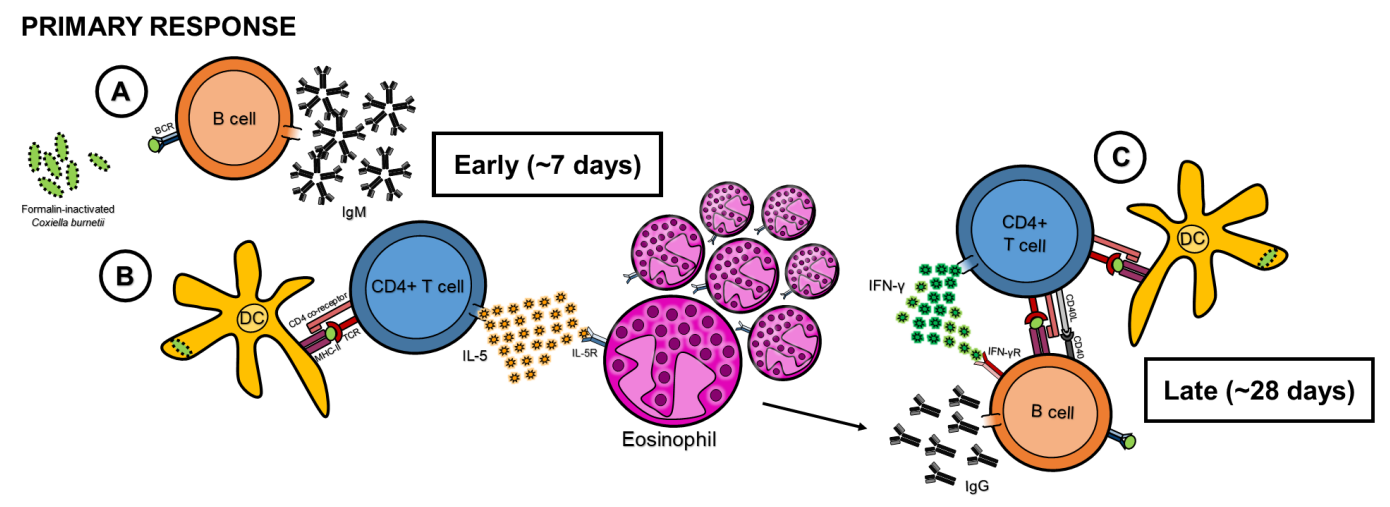

SECONDARY RESPONSE

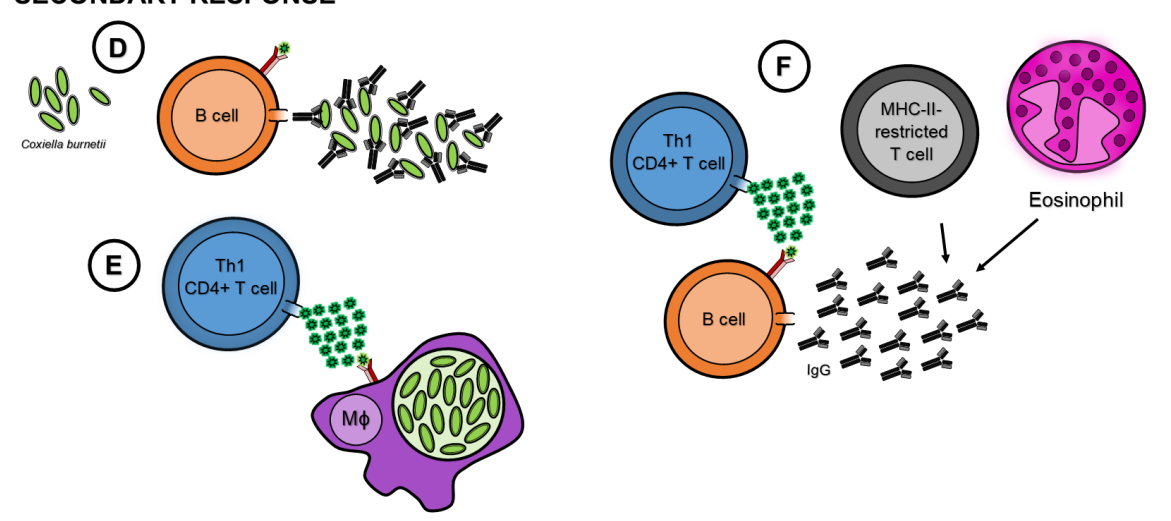

Figure 5-2. A working model of protective immunity

The primary response to vaccination involves early $T$ cell-independent IgM production (A) as well as $\mathrm{CD} 4^{+} \mathrm{T}$ cell-dependent eosinophil recruitment (B). (C) $\mathrm{CD} 4^{+} \mathrm{T}$ cell-dependent IgG production increases over the course of vaccination, peaking at the time of challenge. (D) Following secondary challenge, Coxiella-specific antibodies likely neutralize the majority of incoming bacteria. (E) Cell-mediated immunity is required to clear the remaining intracellular bacteria. (F) Eosinophils and $\mathrm{MHC}$-II-restricted T cells contribute to the secondary antibody response, although the exact mechanism(s) remain unclear. 


\section{Inflammation vs. bacterial clearance}

Inflammation is a balancing act between pathogen clearance and tissue pathology. In my model, the Th1 pathway appears to be involved in the regulation of the inflammatory response. This is evidenced by the observation that PIV-vaccinated CD4 KO (Figures 4-3, 4-5, 4-6, and 4-7), Tbet KO (Figure 4-7), and IFN- $\gamma$ KO mice (Figure 4-8) develop significant splenomegaly compared to PIV-vaccinated WT mice. However, this pathway may be dispensable for bacterial clearance as splenic bacterial loads in vaccinated $\mathrm{CD} 4$, Tbet, and IFN- $\gamma \mathrm{KO}$ mice were equal to vaccinated WT mice. This was unexpected considering one report describing a mortality rate of $75 \%$ following primary infection of IFN- $\gamma \mathrm{KO}$ mice due to uncontrolled bacterial replication (201). Indeed, treatment with IFN- $\gamma$ is required for macrophage microbicidal activity in vitro $(246,281,282)$. However, there are likely redundancies in the immune response which facilitate protection in the absence of IFN- $\gamma$. For example, TNF- $\alpha$ is another proinflammatory cytokine that can activate macrophages (283) and contribute to host immunity against $C$. burnetii infection (201).

While other cytokines are capable of mediating $C$. burnetii clearance in the absence of IFN- $\gamma$, it is likely still a key mediator in healthy patients. Moreover, my data suggests that there is probably a threshold for IFN- $\gamma$, whereby insufficient levels result in uncontrolled bacterial replication and overproduction results in tissue pathology. Following the adoptive transfer of $\mathrm{CD}^{+} \mathrm{T}$ cells from PIVvaccinated WT donors, I found that naïve CD4 KO recipient mice were protected 
similar to PIV-vaccinated WT mice in terms of splenomegaly and bacterial clearance (Figure 4-5). Conversely, the adoptive transfer of $\mathrm{CD}^{+} \mathrm{T}$ cells from naïve WT donors to PIV-vaccinated CD4 KO recipient mice resulted in significantly higher splenomegaly than PIV-vaccinated CD4 KO mice which had not received $\mathrm{CD}^{+} \mathrm{T}$ cells (Figure 4-6). This data led me to hypothesize that $\mathrm{CD} 4^{+} \mathrm{T}$ celldependent IFN- $\gamma$ secretion varies depending on the immune status of both the donor and recipient hosts. To test this, one could restimulate mixed cell cultures ex vivo and evaluate IFN- $\gamma$ production by ELISA. The two key groups to include which would recapitulate the in vivo adoptive transfer groups would be naïve APCs cultured with PIV-vaccinated CD4+ ${ }^{+}$cells and PIV-vaccinated APCs cultured with naïve $\mathrm{CD} 4^{+} \mathrm{T}$ cells. A better understanding of inflammation regulation will be informative for diminishing vaccine reactions and enhancing protective responses.

\section{Reducing vaccine reactions}

One of the largest hindrances to regulatory approval of a $Q$ fever vaccine in the United States is the high incidence of adverse reactions in vaccine recipients. These granulomatous or DTH reactions $(97,132,204,284-288)$ coincide with the development of CMI (289), thus complicating efforts to develop a protective nonreactogenic $Q$ fever vaccine. DTH reactions occur when $C D 4^{+} T$ cells recognize foreign antigens via MHC-II-restricted antigen presentation and consequently stimulate cytokine-mediated inflammation (290). I've shown that CD4 ${ }^{+} \mathrm{T}$ cells only partially contribute to the MHC-II-restricted protective response, as vaccinated CD4 KO mice have intermediate splenomegaly compared to vaccinated WT and 
MHC-II KO mice (Figure 4-3B). Moreover, vaccinated CD4 KO mice had equal or better bacterial clearance when compared to vaccinated WT mice (Figures 4-3, 45, 4-6, and 4-7). This data suggests that perhaps future $Q$ fever vaccines could modulate the $\mathrm{CD}^{+} \mathrm{T}$ cell response to reduce inflammation without sacrificing protection.

Furthermore, the nonclassical MHC-II-restricted T cell populations discovered in Chapter 4 could serve as potential alternative targets for a new $Q$ fever vaccine. Taking a systems vaccinology approach, one could use immunoinformatics to identify proteins that are recognized by MHC-II and stimulate a proliferative response ex vivo in T cells from CD4 KO mice. This would generate potential MHCII-restricted epitope leads that would, in theory, target these nonclassical pathways. It would be important to cross-reference these leads with those generated in a WT system as you would want to eliminate those which also activate $\mathrm{CD}^{+} \mathrm{T}$ cells, if possible. While these populations are normally in low abundance (261), infection with other intracellular pathogens (e.g. L. major) stimulates their expansion in a WT host (290) which indicates that they are not an artifact of CD4 deficiency. One caveat to this approach is the potential that the expansion of these cells would elicit inflammation similar to $\mathrm{CD} 4^{+} \mathrm{T}$ cells.

Reducing the dose of vaccine antigens delivered (i.e. dose sparing) is another approach that may reduce vaccine reactions. The potential success of this approach is supported by a study from Fries and colleagues who demonstrated that the degree of vaccine reactogenicity correlated with the antigen dose delivered 
(207). The addition of a vaccine adjuvant would maximize the effectiveness of this approach by maintaining, or even enhancing, protective immunity as the antigen dose decreases.

\section{Boosting innate immunity through adjuvant selection}

In Chapter 3, I discovered that eosinophils are recruited to the spleens of PIVvaccinated mice within a week of vaccination (Figure 3-5). This was a novel finding considering eosinophils are classically associated with parasitic diseases and allergic airway inflammation. Eosinophil accumulation was $\mathrm{CD} 4^{+} \mathrm{T}$ cell-dependent (Figure 3-6) and eosinophil deficiency resulted in an early defect in vaccine protection (Figure 3-7) which was associated with alterations in the Coxiellaspecific antibody response (Figures 3-7 and 3-8). Overall, this led to the conclusion that eosinophils contribute to antibody isotype switching and may partially contribute to early vaccine-induced protective immunity against $C$. burnetii. This breakthrough has implications for vaccine development as it serves as a target for adjuvant selection.

Adjuvants are immune-stimulating agents added to vaccines to enhance immunity and their effects are typically mediated by PRR signaling. Monophosphoryl lipid A (MPL), for example, is an adjuvant which elicits a Th1 profile through activation of the TLR4/TRIF pathway $(291,292)$. DCs are professional APCs capable of integrating TLR signaling with MHC-II-restricted antigen presentation to activate $\mathrm{CD} 4^{+} \mathrm{T}$ cells. Accumulating evidence suggests that $\mathrm{CD} 4^{+} \mathrm{T}$ cells can be skewed depending on which TLR and DC subset are activated (293). In mice, CD8 $\alpha^{+}$DCs 
stimulate Th1 subset CD4+ $4^{+}$cells, while CD8 $\alpha^{-}$DCs stimulate Th2 cells (294, 295). Similarly, human CD11 ${ }^{+}$DCs and CD11C plasmacytoid DCs induce Th1 and Th2 responses, respectively (296). Thus, in addition to targeting specific TLRs, directing vaccine antigens to a particular DC subset could further enhance adaptive immunity.

TLR2 is known to elicit Th2 responses following triggering with a synthetic agonist $(297,298)$ and is, therefore, a potential target that could activate Th2 CD4 ${ }^{+} \mathrm{T}$ cells for the recruitment of eosinophils. Furthermore, there is precedent in the literature that TLR2 contributes to host immunity against $C$. burnetii (299-302). TLR2 activation by $C$. burnetii NMII is critical for intracellular growth restriction in vitro (299). Additionally, TLR2 expression on murine hematopoietic cells was shown to be critical for bacterial growth restriction in the lung following i.t. challenge with $C$. burnetii NMI (302). Identifying which $C$. burnetii epitopes stimulate TLR2 may facilitate the design of a TLR2-targeting adjuvant derived from the bacterium itself. This approach was successfully applied to the design of a TLR2 agonist which enhanced the efficacy of an acellular vaccine against Bordetella pertussis (191). Dunne et al. identified a $B$. pertussis lipoprotein which activated TLR2 and subsequently synthesized a mimetic peptide that enhanced Th1, Th17, and IgG2a responses. Of note, these responses were superior to those achieved with an alum adjuvant.

Alternatively, MF59 is an oil-in-water emulsion which also stimulates superior antibody responses to alum and elicits long-lasting CMI (292, 303). This 
commercially-available adjuvant increases antigen uptake by dendritic cells (304) and promotes the recruitment of $\mathrm{CD} 11 \mathrm{~b}^{+}$and $\mathrm{MHCll}^{+}$cells to the injection site (305). When formulated into the seasonal flu vaccine, MF59 reduced the number of doses required for seroconversion (306), thus supporting the dose sparing approach discussed previously. Furthermore, MF59-adjuvanted flu vaccines were immunogenic in children (307) and the elderly (306). As discussed in Chapter 3, these groups are often vaccine hypo- or non-responders. This is especially the case for vaccines containing polysaccharide antigens such as the pneumococcal vaccine (Pneumovax ${ }^{\circledR}$ ) and $\mathrm{Q}-\mathrm{VAX}{ }^{\circledR}$, in which C. burnetii LPS contributes to protection. As such, an adjuvant that can stimulate immunity in these groups is desirable.

\section{Targeting T cells with epitope selection}

Most vaccines are capable of eliciting a strong antibody response, however, generating long-lived $\mathrm{CMI}$ is more challenging. Here, I discovered that vaccineinduced protective immunity against $C$. burnetii requires MHC-II. This differs from host defense against primary infection, which relies heavily on MHC-I-dependent mechanisms (175). While MHC-II-dependent responses are typically CD4+ $\mathrm{T}$ cellmediated, the data presented here suggest that $\mathrm{CD} 4^{+} \mathrm{T}$ cells only play a partial role in vaccine-induced protective immunity against $C$. burnetii (Figure 4-3). Instead, there appear to be $\mathrm{MHC}$-II-restricted $\mathrm{CD}^{+} \mathrm{T}$ cell-independent mechanisms that are sufficient for generating protection in the absence of CD4. 
This discovery highlights a pathway that future vaccines could target to elicit protection without causing CD4+ $\mathrm{T}$ cell-dependent $\mathrm{DTH}$ reactions.

As part of the Q-vaxcelerate consortium, Scholzen et al. recently published work describing an immunoinformatic screen that predicted MHC-II-restricted epitopes conserved across $C$. burnetii strains (151). $75 \%$ of their predicted MHC-II epitopes bound MHC-II in a human leukocyte antigen (HLA) binding assay, indicating the robustness of this method. Furthermore, selected epitopes elicited an IFN- $\gamma$ response in humanized MHC-II transgenic mice. Perhaps the most exciting finding in this study was that a portion of their epitope leads generated IFN- $\gamma$ recall responses in human peripheral blood mononuclear cells (PBMCs) from individuals previously exposed to $C$. burnetii during the Netherlands outbreak. These epitopes were primarily derived from an immunodominant pool, meaning they are capable of eliciting an antibody response. While these data are encouraging, it remains to be determined if a multi-epitope vaccine based on this screen will be successful considering the failure of previous attempts at a subunit vaccine against $Q$ fever. Ultimately, incorporating the right innate immune-stimulating adjuvant with MHCII-restricted epitopes should result in a highly protective $Q$ fever vaccine. 


\section{REFERENCES}

1. Derrick EH. 1937. "Q" fever, a new fever entity: clinical features, diagnosis and laboratory investigation. Med J Aust 2:281-299.

2. Derrick EH. 1983. "Q" fever, a new fever entity: clinical features, diagnosis and laboratory investigation. Rev Infect Dis 5:790-800.

3. Burnet FM, Freeman M. 1937. Experimental studies on the virus of "Q" fever. Med J Aust 2:299-305.

4. Burnet FM, Freeman M. 1983. Experimental studies on the virus of "Q" fever. Rev Infect Dis 5:800-808.

5. Davis GE, Cox HR, Parker RR, Dyer RE. 1938. A filter-passing infectious agent isolated from ticks. Pub Health Rep 53.

6. Dyer RE. 1938. A filter-passing infectious agent isolated from ticks. IV. Human infection. Pub Health Rep 53:2277-2282.

7. McCaul TF, Williams JC. 1981. Developmental cycle of Coxiella burnetii: structure and morphogenesis of vegetative and sporogenic differentiations. J Bacteriol 147:1063-1076.

8. Roest HI, Bossers A, van Zijderveld FG, Rebel JM. 2013. Clinical microbiology of Coxiella burnetii and relevant aspects for the diagnosis and control of the zoonotic disease $Q$ fever. Vet $Q$ 33:148-160.

9. Mertens K, Samuel J. 2007. Bacteriology of Coxiella, p 257-270. In Raoult D, Parola P (ed), Rickettsial Diseases. Taylor \& Francis Group, LLC.

10. Angelakis E, Raoult D. 2010. Q fever. Vet Microbiol 140:297-309. 
11. Kersh GJ, Fitzpatrick KA, Self JS, Priestley RA, Kelly AJ, Lash RR, Marsden-Haug N, Nett RJ, Bjork A, Massung RF, Anderson AD. 2013. Presence and persistence of Coxiella burnetii in the environments of goat farms associated with a $Q$ fever outbreak. Appl Environ Microbiol 79:16971703.

12. Calverley M, Erickson S, Read AJ, Harmsen AG. 2012. Resident alveolar macrophages are susceptible to and permissive of Coxiella burnetii infection. PLoS One 7:e51941.

13. Graham JG, MacDonald LJ, Hussain SK, Sharma UM, Kurten RC, Voth DE. 2013. Virulent Coxiella burnetii pathotypes productively infect primary human alveolar macrophages. Cell Microbiol 15:1012-1025.

14. Kazar J, Skultetyova E, Brezina R. 1975. Phagocytosis of Coxiella burnetii by macrophages. Acta Virol 19:426-431.

15. Shannon JG, Howe D, Heinzen RA. 2005. Virulent Coxiella burnetii does not activate human dendritic cells: role of lipopolysaccharide as a shielding molecule. Proc Natl Acad Sci U S A 102:8722-8727.

16. Elliott A, Peng Y, Zhang G. 2013. Coxiella burnetii interaction with neutrophils and macrophages in vitro and in SCID mice following aerosol infection. Infect Immun 81:4604-4614.

17. Schoenlaub L, Elliott A, Freches D, Mitchell WJ, Zhang G. 2015. Role of B cells in host defense against primary Coxiella burnetii infection. Infect Immun 83:4826-4836. 
18. Uribe-Querol E, Rosales C. 2017. Control of phagocytosis by microbial pathogens. Front Immunol 8.

19. Voth DE, Heinzen RA. 2007. Lounging in a lysosome: the intracellular lifestyle of Coxiella burnetii. Cell Microbiol 9:829-840.

20. Voth DE, Heinzen RA. 2009. Coxiella type IV secretion and cellular microbiology. Curr Opin Microbiol 12:74-80.

21. Beare PA, Gilk SD, Larson CL, Hill J, Stead CM, Omsland A, Cockrell DC, Howe D, Voth DE, Heinzen RA. 2011. Dot/lcm type IVB secretion system requirements for Coxiella burnetii growth in human macrophages. MBio 2:e00175-11.

22. Winchell CG, Graham JG, Kurten RC, Voth DE. 2014. Coxiella burnetii type IV secretion-dependent recruitment of macrophage autophagosomes. Infect Immun 82:2229-2238.

23. Larson CL, Beare PA, Voth DE, Howe D, Cockrell DC, Bastidas RJ, Valdivia $\mathrm{RH}$, Heinzen RA. 2015. Coxiella burnetii effector proteins that localize to the parasitophorous vacuole membrane promote intracellular replication. Infect Immun 83:661-670.

24. Mahapatra S, Gallaher B, Smith SC, Graham JG, Voth DE, Shaw El. 2016. Coxiella burnetii employs the Dot/lcm type IV secretion system to modulate host NF-kappaB/RelA activation. Front Cell Infect Microbiol 6:188. 
25. Amano K, Williams JC, Missler SR, Reinhold VN. 1987. Structure and biological relationships of Coxiella burnetii lipopolysaccharides. J Biol Chem 262:4740-4747.

26. Barry Abdoulaye O, Boucherit N, Mottola G, Vadovic P, Trouplin V, Soubeyran P, Capo C, Bonatti S, Nebreda A, Toman R, Lemichez E, Mege J-L, Ghigo E. 2012. Impaired stimulation of p38a-MAPK/Nps41-HOPS by LPS from pathogenic Coxiella burnetii prevents trafficking to microbicidal phagolysosomes. Cell Host Microbe 12:751-763.

27. Baca OG, Paretsky D. 1983. Q fever and Coxiella burnetii: a model for hostparasite interactions. Microbiol Rev 47:127-149.

28. Hackstadt T, Peacock MG, Hitchcock PJ, Cole RL. 1985. Lipopolysaccharide variation in Coxiella burnetii: intrastrain heterogeneity in structure and antigenicity. Infect Immun 48:359-365.

29. Kersh GJ, Oliver LD, Self JS, Fitzpatrick KA, Massung RF. 2011. Virulence of pathogenic Coxiella burnetii strains after growth in the absence of host cells. Vector Borne Zoonotic Dis 11:1433-1438.

30. Hoover TA, Culp DW, Vodkin MH, Williams JC, Thompson HA. 2002. Chromosomal DNA deletions explain phenotypic characteristics of two antigenic variants, phase II and RSA 514 (crazy), of the Coxiella burnetii Nine Mile strain. Infect Immun 70:6726-6733. 
31. Moos A, Hackstadt T. 1987. Comparative virulence of intra- and interstrain lipopolysaccharide variants of Coxiella burnetii in the guinea pig model. Infect Immun 55:1144-1150.

32. Williams JC, Thompson HA. 1991. Infectivity, virulence, and pathogenicity of Coxiella burnetii for various hosts, p 21-71, Q Fever: The Biology of Coxiella burnetii, vol 2. CRC Press.

33. Luoto L, Huebner RJ. 1950. Q fever studies in southern California; IX. Isolation of $\mathrm{Q}$ fever organisms from parturient placentas of naturally infected dairy cows. Public Health Rep 65:541-544.

34. Welsh HH, Lennette EH, Abinanti FR, Winn JF. 1951. Q fever in California. IV. Occurrence of Coxiella burnetii in the placenta of naturally infected sheep. Public Health Rep 66:1473-1477.

35. Marmion BP, Ormsbee RA, Kyrkou M, Wright J, Worswick D, Cameron S, Esterman A, Feery B, Collins W. 1984. Vaccine prophylaxis of abattoirassociated $Q$ fever. Lancet 2:1411-1414.

36. McQuiston JH, Holman RC, McCall CL, Childs JE, Swerdlow DL, Thompson HA. 2006. National surveillance and the epidemiology of human $Q$ fever in the United States, 1978-2004. Am J Trop Med Hyg 75:36-40.

37. Whitney EA, Massung RF, Candee AJ, Ailes EC, Myers LM, Patterson NE, Berkelman RL. 2009. Seroepidemiologic and occupational risk survey for Coxiella burnetii antibodies among US veterinarians. Clin Infect Dis 48:550557. 
38. Enserink M. 2010. Infectious diseases. Questions abound in Q-fever explosion in the Netherlands. Science 327:266-267.

39. Roest HI, Tilburg JJ, van der Hoek W, Vellema P, van Zijderveld FG, Klaassen $\mathrm{CH}$, Raoult D. 2011. The Q fever epidemic in the Netherlands: history, onset, response and reflection. Epidemiol Infect 139:1-12.

40. van der Hoek W, Morroy G, Renders NH, Wever PC, Hermans MH, Leenders AC, Schneeberger PM. 2012. Epidemic Q fever in humans in the Netherlands. In Toman R, Heinzen RA, Samuel JE, Mege JL (ed), Coxiella burnetii: recent advances and new perspectives in research of the $Q$ fever bacterium doi:10.1007/978-94-007-4315-1. Springer Netherlands

41. Eldin C, Melenotte C, Mediannikov O, Ghigo E, Million M, Edouard S, Mege JL, Maurin M, Raoult D. 2017. From Q fever to Coxiella burnetii infection: a paradigm change. Clin Microbiol Rev 30:115-190.

42. Buhariwalla F, Cann B, Marrie TJ. 1996. A dog-related outbreak of Q fever. Clin Infect Dis 23:753-755.

43. Davoust B, Marié J-L, Pommier de Santi V, Berenger J-M, Edouard S, Raoult D. 2014. Three-toed sloth as putative reservoir of Coxiella burnetii, Cayenne, French Guiana. Emerg Infect Dis 20:1760-1761.

44. Biberstein EL, Behymer DE, Bushnell R, Crenshaw G, Riemann HP, Franti CE. 1974. A survey of $Q$ fever (Coxiella burnetii) in California dairy cows. Am J Vet Res 35:1577-1582. 
45. Kim SG, Kim EH, Lafferty CJ, Dubovi E. 2005. Coxiella burnetii in bulk tank milk samples, United States. Emerg Infect Dis 11:619-621.

46. Huebner RJ, Jellison WL, Beck MD, Wilcox FP. 1949. Q fever studies in southern California: III. Effects of pasteurization on survival of $C$. burnetii in naturally infected milk. Pub Health Rep 64.

47. Rowbotham TJ. 1980. Preliminary report on the pathogenicity of Legionella pneumophila for freshwater and soil amoebae. J Clin Pathol 33:1179-1183.

48. Maurin M, Raoult D. 1999. Q fever. Clin Microbiol Rev 12:518-553.

49. La Scola B, Raoult D. 2001. Survival of Coxiella burnetii within free-living amoeba Acanthamoeba castellanii. Clin Microbiol Infect 7:75-79.

50. Duron O, Sidi-Boumedine K, Rousset E, Moutailler S, Jourdain E. 2015. The importance of ticks in $Q$ fever transmission: what has (and has not) been demonstrated? Trends Parasitol 31:536-552.

51. Anonymous. 1950. Experimental Q fever in man. Br Med J 1:1000.

52. Milazzo A, Hall R, Storm PA, Harris RJ, Winslow W, Marmion BP. 2001. Sexually transmitted Q fever. Clin Infect Dis 33:399-402.

53. Racult D, Stein A. 1994. Q fever during pregnancy--a risk for women, fetuses, and obstetricians. N Engl J Med 330:371.

54. Kersh GJ, Priestley R, Massung RF. 2013. Stability of Coxiella burnetii in stored human blood. Transfusion 53:1493-1496. 
55. Slot E, Hogema BM, Molier M, Zaaijer HL. 2014. Screening of blood donors for chronic Coxiella burnetii infection after large $Q$ fever outbreaks. Transfusion 54:2867-2870.

56. Oei W, Kretzschmar MEE, Zaaijer HL, Coutinho R, van der Poel CL, Janssen MP. 2014. Estimating the transfusion transmission risk of $Q$ fever. Transfusion 54:1705-1711.

57. Madariaga MG, Rezai K, Trenholme GM, Weinstein RA. 2003. Q fever: a biological weapon in your backyard. Lancet Infect Dis 3:709-721.

58. Oyston PC, Davies C. 2011. Q fever: the neglected biothreat agent. J Med Microbiol 60:9-21.

59. Brooke RJ, Kretzschmar ME, Mutters NT, Teunis PF. 2013. Human dose response relation for airborne exposure to Coxiella burnetii. BMC Infect Dis 13:488.

60. Hawker JI, Ayres JG, Blair I, Evans MR, Smith DL, Smith EG, Burge PS, Carpenter MJ, Caul EO, Coupland B, Desselberger U, Farrell ID, Saunders PJ, Wood MJ. 1998. A large outbreak of Q fever in the West Midlands: windborne spread into a metropolitan area? Commun Dis Public Health 1:180-187.

61. Tissot-Dupont H, Torres S, Nezri M, Raoult D. 1999. Hyperendemic focus of $Q$ fever related to sheep and wind. Am J Epidemiol 150:67-74.

62. Tissot-Dupont H, Amadei MA, Nezri M, Raoult D. 2004. Wind in November, Q fever in December. Emerg Infect Dis 10:1264-1269. 
63. CDC. 2019. Q fever, on Centers for Disease Control and Prevention. https://www.cdc.gov/gfever/. Accessed

64. Raoult D, Marrie T, Mege J. 2005. Natural history and pathophysiology of Q fever. Lancet Infect Dis 5:219-226.

65. Parker NR, Barralet JH, Bell AM. 2006. Q fever. Lancet 367:679-688.

66. Million M, Raoult D. 2017. No such thing as chronic $Q$ fever. Emerg Infect Dis 23.

67. Tissot-Dupont H, Vaillant V, Rey S, Raoult D. 2007. Role of sex, age, previous valve lesion, and pregnancy in the clinical expression and outcome of $Q$ fever after a large outbreak. Clin Infect Dis 44:232-237.

68. Raoult D, Fenollar F, Stein A. 2002. Q fever during pregnancy: diagnosis, treatment, and follow-up. Arch Intern Med 162:701-704.

69. Hatchette TF, Hayes M, Merry H, Schlech WF, Marrie TJ. 2003. The effect of $C$. burnetii infection on the quality of life of patients following an outbreak of $Q$ fever. Epidemiol Infect 130:491-495.

70. Limonard GJ, Peters JB, Nabuurs-Franssen MH, Weers-Pothoff G, Besselink R, Groot CA, Dekhuijzen PN, Vercoulen JH. 2010. Detailed analysis of health status of $Q$ fever patients 1 year after the first Dutch outbreak: a case-control study. Q J Med 103:953-958.

71. Hussain-Yusuf H, Islam A, Healy B, Lockhart M, Nguyen C, Sukocheva O, Stenos J, Graves S. 2012. An analysis of Q fever patients 6 years after an outbreak in Newport, Wales, UK. Q J Med 105:1067-1073. 
72. van Loenhout JA, Hautvast JL, Vercoulen JH, Akkermans RP, Wijkmans CJ, van der Velden K, Paget WJ. 2015. Q-fever patients suffer from impaired health status long after the acute phase of the illness: results from a 24-month cohort study. J Infect 70:237-246.

73. Morroy G, Van Der Hoek W, Nanver ZD, Schneeberger PM, Bleeker-Rovers CP, Van Der Velden J, Coutinho RA. 2016. The health status of a village population, 7 years after a major $Q$ fever outbreak. Epidemiol Infect 144:1153-1162.

74. Limonard GJ, Peters JB, Besselink R, Groot CA, Dekhuijzen PN, Vercoulen JH, Nabuurs-Franssen MH. 2016. Persistence of impaired health status of Q fever patients 4 years after the first Dutch outbreak. Epidemiol Infect 144:1142-1147.

75. Marmion BP, Sukocheva O, Storm PA, Lockhart M, Turra M, Kok T, Ayres J, Routledge H, Graves S. 2009. Q fever: persistence of antigenic nonviable cell residues of Coxiella burnetii in the host--implications for post $\mathrm{Q}$ fever infection fatigue syndrome and other chronic sequelae. Q J Med 102:673-684.

76. Sukocheva OA, Marmion BP, Storm PA, Lockhart M, Turra M, Graves S. 2010. Long-term persistence after acute $Q$ fever of non-infective Coxiella burnetii cell components, including antigens. Q J Med 103:847-863.

77. Mayo. 2019. [Test Summary] Q Fever Antibody, IgG and IgM, Serum. https://www. mayocliniclabs.com/test-catalog/Overview/83149. Accessed 
78. Mayo. 2019. [Test Summary] Coxiella burnetii (Q Fever), Molecular Detection, PCR, Serum. https://www. mayocliniclabs.com/testcatalog/Overview/62194. Accessed

79. Mayo. 2019. [Test Summary] Coxiella burnetii ( $Q$ fever), Molecular Detection, PCR, Varies. https://www. mayocliniclabs.com/testcatalog/Overview/62193. Accessed

80. Mayo. 2019. [Test Summary] Coxiella burnetii ( $Q$ fever), Molecular Detection, PCR, Blood. https://www. mayocliniclabs.com/testcatalog/Overview/62248. Accessed

81. Rolain JM, Boulos A, Mallet MN, Raoult D. 2005. Correlation between ratio of serum doxycycline concentration to MIC and rapid decline of antibody levels during treatment of $Q$ fever endocarditis. Antimicrob Agents Chemother 49:2673-2676.

82. Rolain JM. 2005. Activity of telithromycin against thirteen new isolates of $C$. burnetii including three resistant to doxycycline. Ann $N$ Y Acad Sci 1063:252-256.

83. Rouli L, Rolain JM, El Filali A, Robert C, Raoult D. 2012. Genome sequence of Coxiella burnetii 109, a doxycycline-resistant clinical isolate. J Bacteriol 194:6939.

84. Boulos A, Rolain JM, Maurin M, Raoult D. 2004. Measurement of the antibiotic susceptibility of Coxiella burnetii using real time PCR. Int $\mathrm{J}$ Antimicrob Agents 23:169-174. 
85. Musso D, Drancourt M, Osscini S, Raoult D. 1996. Sequence of quinolone resistance-determining region of gyrA gene for clinical isolates and for an in vitro-selected quinolone-resistant strain of Coxiella burnetii. Antimicrob Agents Chemother 40:870-873.

86. Spyridaki I, Psaroulaki A, Aransay A, Scoulica E, Tselentis Y. 2000. Diagnosis of quinolone-resistant Coxiella burnetii strains by PCR-RFLP. J Clin Lab Anal 14:59-63.

87. Eldin C, Perreal C, Mahamat A, Djossou F, Edouard S, Raoult D. 2015. Antibiotic susceptibility determination for six strains of Coxiella burnetii MST 17 from Cayenne, French Guiana. Int J Antimicrob Agents 46:600-602.

88. Raoult D, Houpikian P, Tissot Dupont H, Riss JM, Arditi-Djiane J, Brouqui P. 1999. Treatment of $Q$ fever endocarditis: comparison of 2 regimens containing doxycycline and ofloxacin or hydroxychloroquine. Arch Intern Med 159:167-173.

89. Brennan RE, Samuel JE. 2003. Evaluation of Coxiella burnetii antibiotic susceptibilities by real-time PCR assay. J Clin Microbiol 41:1869-1874.

90. Raoult D, Drancourt M, Vestris G. 1990. Bactericidal effect of doxycycline associated with lysosomotropic agents on Coxiella burnetii in P388D1 cells. Antimicrob Agents Chemother 34:1512-1514.

91. Bengtson IA. 1941. Studies on active and passive immunity in "Q" fever infected and immunized guinea pigs. Pub Health Rep 56:327-345. 
92. Smadel JE, Snyder MJ, Robbins FC. 1948. Vaccination against $Q$ fever. Am J Hyg 47:71-81.

93. Berman S, Cole G, Lowenthal JP, Gochenour RB. 1960. Safety test for Q fever vaccine. J Bacteriol 79:747-751.

94. Anacker RL, Lackman DB, Pickens EG, Ribi E. 1962. Antigenic and skinreactive properties of fractions of Coxiella burnetii. J Immunol 89:145-153.

95. Ormsbee RA, Bell EJ, Lackman DB, Tallent G. 1964. The influence of phase on the protective potency of Q fever vaccine. J Immunol 92:404-412.

96. Baca OG, Paretsky D. 1974. Some physiological and biochemical effects of a Coxiella burnetii lipopolysaccharide preparation on guinea pigs. Infect Immun 9:939-945.

97. Heggers JP, Mallavia LP, Hinrichs DJ. 1974. The cellular immune response to antigens of Coxiella burnetii. Can J Microbiol 20:657-662.

98. Kishimoto RA, Burger GT. 1977. Appearance of cellular and humoral immunity in guinea pigs after infection with Coxiella burnetii administered in small-particle aerosols. Infect Immun 16:518-521.

99. Waag DM, England MJ, Pitt ML. 1997. Comparative efficacy of a Coxiella burnetii chloroform:methanol residue (CMR) vaccine and a licensed cellular vaccine (Q-Vax) in rodents challenged by aerosol. Vaccine 15:1779-1783.

100. Russell-Lodrigue KE, Andoh M, Poels MW, Shive HR, Weeks BR, Zhang GQ, Tersteeg C, Masegi T, Hotta A, Yamaguchi T, Fukushi H, Hirai K, McMurray DN, Samuel JE. 2009. Coxiella burnetii isolates cause 
genogroup-specific virulence in mouse and guinea pig models of acute $Q$ fever. Infect Immun 77:5640-5650.

101. Long CM, Beare PA, Cockrell DC, Larson CL, Heinzen RA. 2019. Comparative virulence of diverse Coxiella burnetii strains. Virulence 10:133-150.

102. Hackstadt T. 1986. Antigenic variation in the phase I lipopolysaccharide of Coxiella burnetii isolates. Infect Immun 52:337-340.

103. Samuel JE, Frazier ME, Mallavia LP. 1985. Correlation of plasmid type and disease caused by Coxiella burnetii. Infect Immun 49:775-779.

104. Hendrix LR, Samuel JE, Mallavia LP. 1991. Differentiation of Coxiella burnetii isolates by analysis of restriction-endonuclease-digested DNA separated by SDS-PAGE. J Gen Microbiol 137:269-276.

105. Gonder JC, Kishimoto RA, Kastello MD, Pedersen CE, Jr., Larson EW. 1979. Cynomolgus monkey model for experimental $Q$ fever infection. J Infect Dis 139:191-196.

106. Waag DM, Byrne WR, Estep J, Gibbs P, Pitt ML, Banfield CM. 1999. Evaluation of cynomolgus (Macaca fascicularis) and rhesus (Macaca mulatta) monkeys as experimental models of acute $Q$ fever after aerosol exposure to phase-I Coxiella burnetii. Lab Anim Sci 49:634-638.

107. Kishimoto RA, Gonder JC, Johnson JW, Reynolds JA, Larson EW. 1981. Evaluation of a killed phase I Coxiella burnetii vaccine in cynomolgus monkeys (Macaca fascicularis). Lab Anim Sci 31:48-51. 
108. Waag DM, England MJ, Tammariello RF, Byrne WR, Gibbs P, Banfield CM, Pitt ML. 2002. Comparative efficacy and immunogenicity of $Q$ fever chloroform:methanol residue (CMR) and phase I cellular (Q-Vax) vaccines in cynomolgus monkeys challenged by aerosol. Vaccine 20:2623-2634.

109. Scott GH, Williams JC, Stephenson EH. 1987. Animal models in Q fever: pathological responses of inbred mice to phase I Coxiella burnetii. J Gen Microbiol 133:691-700.

110. Bewley KR. 2013. Animal models of $Q$ fever (Coxiella burnetii). Comp Med 63:469-476.

111. Marrie TJ, Stein A, Janigan D, Raoult D. 1996. Route of infection determines the clinical manifestations of acute Q fever. J Infect Dis 173:484487.

112. Melenotte C, Lepidi H, Nappez C, Bechah Y, Audoly G, Terras J, Raoult D, Bregeon F. 2016. Mouse model of Coxiella burnetii aerosolization. Infect Immun 84:2116-2123.

113. Gregory AE, van Schaik EJ, Russell-Lodrigue K, Fratzke A, Samuel JE. 2019. A Coxiella burnetii intratracheal aerosol infection model in mice, guinea pigs and non-human primates. Infect Immun doi:10.1128/iai.0017819.

114. Williams JC, Cantrell JL. 1982. Biological and immunological properties of Coxiella burnetii vaccines in C57BL/10ScN endotoxin-nonresponder mice. Infect Immun 35:1091-1102. 
115. Williams JC, Damrow TA, Waag DM, Amano K. 1986. Characterization of a phase I Coxiella burnetii chloroform-methanol residue vaccine that induces active immunity against $Q$ fever in C57BL/10ScN mice. Infect Immun $51: 851-858$

116. Williams JC, Hoover TA, Waag DM, Banerjee-Bhatnagar N, Bolt CR, Scott GH. 1990. Antigenic structure of Coxiella burnetii. A comparison of lipopolysaccharide and protein antigens as vaccines against $Q$ fever. Ann N Y Acad Sci 590:370-380.

117. Zhang G, Russell-Lodrigue KE, Andoh M, Zhang Y, Hendrix LR, Samuel JE. 2007. Mechanisms of vaccine-induced protective immunity against Coxiella burnetii infection in BALB/c mice. J Immunol 179:8372-8380.

118. Peng Y, Zhang Y, Mitchell WJ, Zhang G. 2012. Development of a lipopolysaccharide-targeted peptide mimic vaccine against $Q$ fever. J Immunol 189:4909-4920.

119. Zhang G, Peng Y, Schoenlaub L, Elliott A, Mitchell W, Zhang Y. 2013. Formalin-inactivated Coxiella burnetii phase I vaccine-induced protection depends on B cells to produce protective IgM and IgG. Infect Immun $81: 2112-2122$.

120. Peng Y, Schoenlaub L, Elliott A, Mitchell WJ, Zhang G. 2014. Characterization of a lipopolysaccharide-targeted monoclonal antibody and its variable fragments as candidates for prophylaxis against the obligate 
intracellular bacterial pathogen Coxiella burnetii. Infect Immun 82:45304541.

121. Xiong X, Qi Y, Jiao J, Gong W, Duan C, Wen B. 2014. Exploratory study on Th1 epitope-induced protective immunity against Coxiella burnetii infection. PLoS One 9:e87206.

122. Damrow TA, Williams JC, Waag DM. 1985. Suppression of in vitro lymphocyte proliferation in C57BL/10ScN mice vaccinated with phase I Coxiella burnetii. Infect Immun 47:149-156.

123. Williams JC, Sanchez V, Scott GH, Stephenson EH, Gibbs PH. 1985. Variation in responsiveness of $B A L B / c$ sublines and congenic mice to phase I Coxiella burnetii infection and vaccination, p 189-199. In Potter M (ed), The BALB/c Mouse doi:10.1007/978-3-642-70740-7. Springer, Berlin, Heidelberg.

124. Zhang J, Wen B, Chen M, Zhang J, Niu D. 2005. Balb/c mouse model and real-time quantitative polymerase chain reaction for evaluation of the immunoprotectivity against Q fever. Ann N Y Acad Sci 1063:171-175.

125. Wei $Y$, Wang X, Xiong X, Wen B. 2011. Coxiella burnetii antigen-stimulated dendritic cells mediated protection against Coxiella burnetii in BALB/c mice. J Infect Dis 203:283-291.

126. Feng J, Hu X, Fu M, Dai L, Yu Y, Luo W, Zhao Z, Lu Z, Du Z, Zhou D, Wen B, Jiao J, Xiong X. 2019. Enhanced protection against $Q$ fever in BALB/c mice elicited by immunization of chloroform-methanol residue of Coxiella 
burnetii via intratracheal inoculation. Vaccine doi:10.1016/j.vaccine.2019.08.041.

127. Shannon JG, Cockrell DC, Takahashi K, Stahl GL, Heinzen RA. 2009. Antibody-mediated immunity to the obligate intracellular bacterial pathogen Coxiella burnetii is Fc receptor- and complement-independent. BMC Immunol 10:26.

128. Chen C, van Schaik EJ, Gregory AE, Vigil A, Felgner PL, Hendrix LR, Samuel JE. 2019. Chemokine receptor 7 is essential for Coxiella burnetii whole-cell vaccine induced cellular immunity but dispensable for vaccine mediated protective immunity. J Infect Dis doi:10.1093/infdis/jiz146.

129. Zhang G, Kiss K, Seshadri R, Hendrix LR, Samuel JE. 2004. Identification and cloning of immunodominant antigens of Coxiella burnetii. Infect Immun 72:844-852.

130. Meiklejohn G, Lennette EH. 1950. Q fever in California. I. Observations on vaccination of human beings. Am J Hyg 52:54-64.

131. Stoker MG. 1957. Q fever down the drain. Br Med J 1:425-427.

132. Lackman DB, Bell EJ, Bell JF, Pickens EG. 1962. Intradermal sensitivity testing in man with a purified vaccine for $Q$ fever. Am J Public Health Nations Health 52:87-93.

133. Luoto L, Bell JF, Casey M, Lackman DB. 1963. Q fever vaccination of human volunteers. I. The serologic and skin-test response following subcutaneous injections. Am J Hyg 78:1-15. 
134. Bell JF, Lackman DB, Meis A, Hadlow WJ. 1964. Recurrent reaction of site of $Q$ fever vaccination in a sensitized person. Mil Med 129:591-595.

135. Marmion BP, Ormsbee RA, Kyrkou M, Wright J, Worswick DA, Izzo AA, Esterman A, Feery B, Shapiro RA. 1990. Vaccine prophylaxis of abattoirassociated $Q$ fever: eight years' experience in Australian abattoirs. Epidemiol Infect 104:275-287.

136. Ackland JR, Worswick DA, Marmion BP. 1994. Vaccine prophylaxis of Q fever. A follow-up study of the efficacy of Q-Vax (CSL) 1985-1990. Med J Aust 160:704-708.

137. Zhang G, Samuel JE. 2004. Vaccines against Coxiella infection. Expert Rev Vaccines 3:577-584.

138. Robinson DM, Hasty SE. 1974. Production of a potent vaccine from the attenuated M-44 strain of Coxiella burnetii. Appl Microbiol 27:777-783.

139. Johnson JW, Eddy GA, Pedersen CE, Jr. 1976. Biological properties of the M-44 strain of Coxiella burnetii. J Infect Dis 133:334-338.

140. Johnson JW, McLeod CG, Stookey JL, Higbee GA, Pedersen CE, Jr. 1977. Lesions in guinea pigs infected with Coxiella burnetii strain M-44. J Infect Dis $135: 995-998$.

141. Freylikhman O, Tokarevich N, Suvorov A, Vorobiova E, Totolian A. 2003. Coxiella burnetii persistence in three generations of mice after application of live attenuated human M-44 vaccine against Q fever. Ann N Y Acad Sci 990:496-499. 
142. Mossienko EV, Tokarevich NK, Suvorov AN, Totolian AA. 2003. Detection of Coxiella burnetii by PCR in mice after administration of live M-44 vaccine. Folia Microbiol 48:103-104.

143. Hendrix LR, Mallavia LP, Samuel JE. 1993. Cloning and sequencing of Coxiella burnetii outer membrane protein gene com1. Infect Immun 61:470477.

144. Mo YY, Cianciotto NP, Mallavia LP. 1995. Molecular cloning of a Coxiella burnetii gene encoding a macrophage infectivity potentiator (Mip) analogue. Microbiology 141:2861-2871.

145. Varghees S, Kiss K, Frans G, Braha O, Samuel JE. 2002. Cloning and porin activity of the major outer membrane protein P1 from Coxiella burnetii. Infect Immun 70:6741-6750.

146. Zhang GQ, Samuel JE. 2003. Identification and cloning potentially protective antigens of Coxiella burnetii using sera from mice experimentally infected with Nine Mile phase I. Ann N Y Acad Sci 990:510-520.

147. Zhang YX, Zhi N, Yu SR, Li QJ, Yu GQ, Zhang X. 1994. Protective immunity induced by $67 \mathrm{~K}$ outer membrane protein of phase I Coxiella burnetii in mice and guinea pigs. Acta Virol 38:327-332.

148. FDA. 2019. Animal Rule Summary. https://www.fda.gov/emergencypreparedness-and-response/mom-regulatory-science/animal-rulesummary. Accessed 
149. FDA. 2019. Animal Rule Information. https://www.fda.gov/emergencypreparedness-and-response/mom-regulatory-science/animal-rule-

information. Accessed

150. Reeves PM, Paul SR, Sluder AE, Brauns TA, Poznansky MC. 2017. Qvaxcelerate: a distributed development approach for a new Coxiella burnetii vaccine. Hum Vaccin Immunother 13:2977-2981.

151. Scholzen A, Richard G, Moise L, Baeten LA, Reeves PM, Martin WD, Brauns TA, Boyle CM, Raju Paul S, Bucala R, Bowen RA, Garritsen A, De Groot AS, Sluder AE, Poznansky MC. 2019. Promiscuous Coxiella burnetii CD4 epitope clusters associated with human recall responses are candidates for a novel T-cell targeted multi-epitope $Q$ fever vaccine. Front Immunol 10:207.

152. Scholzen A, Richard G, Moise L, Hartman E, Bleeker-Rovers CP, Reeves PM, Raju Paul S, Martin WD, De Groot AS, Poznansky MC, Sluder AE, Garritsen A. 2019. Coxiella burnetii epitope-specific T-cell responses in patients with chronic $Q$ fever. Infect Immun 87.

153. Hogerwerf L, van den Brom R, Roest HI, Bouma A, Vellema P, Pieterse M, Dercksen D, Nielen M. 2011. Reduction of Coxiella burnetii prevalence by vaccination of goats and sheep, the Netherlands. Emerg Infect Dis 17:379386. 
154. EMA. 2018. [Product Information Sheet] Coxevac®. https://www.ema.europa.eu/en/medicines/veterinary/EPAR/coxevac. Accessed

155. Guatteo R, Seegers H, Joly A, Beaudeau F. 2008. Prevention of Coxiella burnetii shedding in infected dairy herds using a phase I C. burnetii inactivated vaccine. Vaccine 26:4320-4328.

156. Souriau A, Arricau-Bouvery N, Bodier C, Rodolakis A. 2003. Comparison of the efficacy of $Q$ fever vaccines against Coxiella burnetii experimental challenge in pregnant goats. Ann N Y Acad Sci 990:521-523.

157. Arricau-Bouvery N, Souriau A, Bodier C, Dufour P, Rousset E, Rodolakis A. 2005. Effect of vaccination with phase I and phase II Coxiella burnetii vaccines in pregnant goats. Vaccine 23:4392-4402.

158. Astobiza I, Barandika JF, Ruiz-Fons F, Hurtado A, Povedano I, Juste RA, Garcia-Perez AL. 2011. Four-year evaluation of the effect of vaccination against Coxiella burnetii on reduction of animal infection and environmental contamination in a naturally infected dairy sheep flock. Appl Environ Microbiol 77:7405-7407.

159. Astobiza I, Barandika JF, Ruiz-Fons F, Hurtado A, Povedano I, Juste RA, Garcia-Perez AL. 2011. Coxiella burnetii shedding and environmental contamination at lambing in two highly naturally-infected dairy sheep flocks after vaccination. Res Vet Sci 91:e58-e63. 
160. de Cremoux R, Rousset E, Touratier A, Audusseau G, Nicollet P, Ribaud D, David V, Le Pape M. 2012. Assessment of vaccination by a phase I Coxiella burnetii-inactivated vaccine in goat herds in clinical $Q$ fever situation. FEMS Immunol Med Microbiol 64:104-106.

161. Pinero A, Barandika JF, Hurtado A, Garcia-Perez AL. 2014. Progression of Coxiella burnetii infection after implementing a two-year vaccination program in a naturally infected dairy cattle herd. Acta Vet Scand 56:47.

162. Gonzalez-Barrio D, Ortiz JA, Ruiz-Fons F. 2017. Estimating the efficacy of a commercial phase I inactivated vaccine in decreasing the prevalence of Coxiella burnetii infection and shedding in red deer (Cervus elaphus). Front Vet Sci 4:208.

163. Pulendran B, Ahmed R. 2011. Immunological mechanisms of vaccination. Nat Immunol 12:509-517.

164. Lennette EH, Clark WH, Jensen FW, Toomb CJ. 1952. Q fever studies. XV. Development and persistence in man of complement-fixing and agglutinating antibodies to Coxiella burnetii. J Immunol 68:591-598.

165. Biberstein EL, Crenshaw GL, Behymer DE, Franti CE, Bushnell RB, Riemann HP. 1974. Dermal reactions and antibody responses in dairy cows and laboratory animals vaccinated with Coxiella burnetii. Cornell Vet 64:387-406. 
166. Kazar J, Schramek S, Brezina R. 1977. Analysis of antibody response and immunoglobulins in sera of rabbits and guinea pigs immunized with Coxiella burnetii. Acta Virol 21:246-255.

167. Peacock MG, Fiset P, Ormsbee RA, Wisseman CL, Jr. 1979. Antibody response in man following a small intradermal inoculation with Coxiella burnetii phase I vaccine. Acta Virol 23:73-81.

168. Humphres RC, Hinrichs DJ. 1981. Role of antibody in Coxiella burnetii infection. Infect Immun 31:641-645.

169. Worswick D, Marmion BP. 1985. Antibody responses in acute and chronic Q fever and in subjects vaccinated against $Q$ fever. J Med Microbiol 19:281296.

170. Izzo AA, Marmion BP, Worswick DA. 1988. Markers of cell-mediated immunity after vaccination with an inactivated, whole-cell $Q$ fever vaccine. J Infect Dis 157:781-789.

171. Shannon JG, Heinzen RA. 2009. Adaptive immunity to the obligate intracellular pathogen Coxiella burnetii. Immunol Res 43:138-148.

172. Read AJ, Erickson S, Harmsen AG. 2010. Role of CD4+ and CD8+ T cells in clearance of primary pulmonary infection with Coxiella burnetii. Infect Immun 78:3019-3026.

173. Capo C, Mege JL. 2012. Role of innate and adaptive immunity in the control of $Q$ fever. Adv Exp Med Biol 984:273-286. 
174. Zhang G, Zhang Y, Samuel JE. 2012. Components of protective immunity. Adv Exp Med Biol 984:91-104.

175. Buttrum L, Ledbetter L, Cherla R, Zhang Y, Mitchell WJ, Zhang G. 2018. Both major histocompatibility complex class I (MHC-I) and MHC-II molecules are required, while MHC-I appears to play a critical role in host defense against primary Coxiella burnetii infection. Infect Immun 86:e00602-17.

176. Snapper CM, Paul WE. 1987. Interferon-gamma and B cell stimulatory factor-1 reciprocally regulate Ig isotype production. Science 236:944-947.

177. Omsland A, Beare PA, Hill J, Cockrell DC, Howe D, Hansen B, Samuel JE, Heinzen RA. 2011. Isolation from animal tissue and genetic transformation of Coxiella burnetii are facilitated by an improved axenic growth medium. Appl Environ Microbiol 77:3720-3725.

178. Yu C, Cantor AB, Yang H, Browne C, Wells RA, Fujiwara Y, Orkin SH. 2002. Targeted deletion of a high-affinity GATA-binding site in the GATA-1 promoter leads to selective loss of the eosinophil lineage in vivo. J Exp Med 195:1387-1395.

179. Rahemtulla A, Fung-Leung WP, Schilham MW, Kundig TM, Sambhara SR, Narendran A, Arabian A, Wakeham A, Paige CJ, Zinkernagel RM, et al. 1991. Normal development and function of CD8+ cells but markedly decreased helper cell activity in mice lacking CD4. Nature 353:180-184. 
180. Koller BH, Marrack P, Kappler JW, Smithies O. 1990. Normal development of mice deficient in beta $2 \mathrm{M}, \mathrm{MHC}$ class I proteins, and CD8+ $\mathrm{T}$ cells. Science 248:1227-1230.

181. Madsen L, Labrecque N, Engberg J, Dierich A, Svejgaard A, Benoist C, Mathis D, Fugger L. 1999. Mice lacking all conventional MHC class II genes. Proc Natl Acad Sci 96:10338-10343.

182. Finotto S, Neurath MF, Glickman JN, Qin S, Lehr HA, Green FH, Ackerman K, Haley K, Galle PR, Szabo SJ, Drazen JM, De Sanctis GT, Glimcher LH. 2002. Development of spontaneous airway changes consistent with human asthma in mice lacking T-bet. Science 295:336-338.

183. Szabo SJ, Sullivan BM, Stemmann C, Satoskar AR, Sleckman BP, Glimcher LH. 2002. Distinct effects of T-bet in Th1 lineage commitment and IFN-gamma production in CD4 and CD8 T cells. Science 295:338-342.

184. Kaplan MH, Schindler U, Smiley ST, Grusby MJ. 1996. Stat6 is required for mediating responses to IL-4 and for development of Th2 cells. Immunity 4:313-319.

185. Ivanov, II, McKenzie BS, Zhou L, Tadokoro CE, Lepelley A, Lafaille JJ, Cua DJ, Littman DR. 2006. The orphan nuclear receptor RORgammat directs the differentiation program of proinflammatory IL-17+ T helper cells. Cell 126:1121-1133. 
186. Sun Z, Unutmaz D, Zou YR, Sunshine MJ, Pierani A, Brenner-Morton S, Mebius RE, Littman DR. 2000. Requirement for RORgamma in thymocyte survival and lymphoid organ development. Science 288:2369-2373.

187. Dalton DK, Pitts-Meek S, Keshav S, Figari IS, Bradley A, Stewart TA. 1993. Multiple defects of immune cell function in mice with disrupted interferongamma genes. Science 259:1739-1742.

188. Clapp B, Yang X, Thornburg T, Walters N, Pascual DW. 2016. Nasal vaccination stimulates CD8(+) T cells for potent protection against mucosal Brucella melitensis challenge. Immunol Cell Biol 94:496-508.

189. Kersh GJ, Fitzpatrick KA, Self JS, Biggerstaff BJ, Massung RF. 2013. Longterm immune responses to Coxiella burnetii after vaccination. Clin Vaccine Immunol 20:129-133.

190. Surendran N, Sriranganathan N, Boyle SM, Hiltbold EM, Tenpenny N, Walker M, Zimmerman K, Werre S, Witonsky SG. 2013. Protection to respiratory challenge of Brucella abortus strain 2308 in the lung. Vaccine 31:4103-4110.

191. Dunne A, Mielke LA, Allen AC, Sutton CE, Higgs R, Cunningham CC, Higgins SC, Mills KH. 2015. A novel TLR2 agonist from Bordetella pertussis is a potent adjuvant that promotes protective immunity with an acellular pertussis vaccine. Mucosal Immunol 8:607-617.

192. Schaut RG, Grinnage-Pulley TL, Esch KJ, Toepp AJ, Duthie MS, Howard RF, Reed SG, Petersen CA. 2016. Recovery of antigen-specific T cell 
responses from dogs infected with Leishmania (L.) infantum by use of vaccine associated TLR-agonist adjuvant. Vaccine 34:5225-5234.

193. Dowling DJ, Scott EA, Scheid A, Bergelson I, Joshi S, Pietrasanta C, Brightman S, Sanchez-Schmitz G, Van Haren SD, Ninkovic J, Kats D, Guiducci C, de Titta A, Bonner DK, Hirosue S, Swartz MA, Hubbell JA, Levy O. 2017. Toll-like receptor 8 agonist nanoparticles mimic immunomodulating effects of the live BCG vaccine and enhance neonatal innate and adaptive immune responses. J Allergy Clin Immunol 140:13391350.

194. Ko EJ, Lee YT, Lee Y, Kim KH, Kang SM. 2017. Distinct effects of monophosphoryl lipid A, oligodeoxynucleotide CpG, and combination adjuvants on modulating innate and adaptive immune responses to influenza vaccination. Immune Netw 17:326-342.

195. Coler RN, Day TA, Ellis R, Piazza FM, Beckmann AM, Vergara J, Rolf T, Lu L, Alter G, Hokey D, Jayashankar L, Walker R, Snowden MA, Evans T, Ginsberg A, Reed SG, Team T-S. 2018. The TLR-4 agonist adjuvant, GLASE, improves magnitude and quality of immune responses elicited by the ID93 tuberculosis vaccine: first-in-human trial. NPJ Vaccines 3:34.

196. Omsland A, Cockrell DC, Howe D, Fischer ER, Virtaneva K, Sturdevant DE, Porcella SF, Heinzen RA. 2009. Host cell-free growth of the $Q$ fever bacterium Coxiella burnetii. Proc Natl Acad Sci 106:4430-4434. 
197. Seqirus. 2017. [Product Information Sheet] Q-VAX® Q Fever Vaccine and $\begin{array}{llllllll}\text { Q-VAX } & \text { Skin } & \text { Test } & \text { (AUST } & R & 100517 & \text { \& } & 100518) .\end{array}$

\section{https://www.seqirus.com.au/docs/315/57/Q-}

\section{VAX\%20PI v6 18Dec2017.pdf. Accessed}

198. Sanicas M, Rossi S, Giudice GD, Rambe DS. 2015. Safety and mechanism of action of licensed vaccine adjuvants. Int Curr Pharm J 4:420-431.

199. Reichman H, Rozenberg P, Munitz A. 2017. Mouse eosinophils: identification, isolation, and functional analysis. Curr Protoc Immunol 119:14.43.1-14.43.22.

200. Moon BG, Takaki S, Miyake K, Takatsu K. 2004. The role of IL-5 for mature B-1 cells in homeostatic proliferation, cell survival, and Ig production. $J$ Immunol 172:6020-6029.

201. Andoh M, Zhang G, Russell-Lodrigue KE, Shive HR, Weeks BR, Samuel JE. 2007. T cells are essential for bacterial clearance, and gamma interferon, tumor necrosis factor alpha, and B cells are crucial for disease development in Coxiella burnetii infection in mice. Infect Immun 75:32453255.

202. Shamri R, Xenakis JJ, Spencer LA. 2011. Eosinophils in innate immunity: an evolving story. Cell Tissue Res 343:57-83.

203. Tyczka J, Eberling S, Baljer G. 2005. Immunization experiments with recombinant Coxiella burnetii proteins in a murine infection model. Ann $\mathrm{N} \mathrm{Y}$ Acad Sci 1063:143-148. 
204. Baeten LA, Podell BK, Sluder AE, Garritsen A, Bowen RA, Poznansky MC. 2018. Standardized guinea pig model for $Q$ fever vaccine reactogenicity. PLoS One 13:e0205882.

205. Sandoz KM, Beare PA, Cockrell DC, Heinzen RA. 2016. Complementation of arginine auxotrophy for genetic transformation of Coxiella burnetii by use of a defined axenic medium. Appl Environ Microbiol 82:3042-3051.

206. Shapiro RA, Siskind V, Schofield FD, Stallman N, Worswick DA, Marmion BP. 1990. A randomized, controlled, double-blind, cross-over, clinical trial of $Q$ fever vaccine in selected Queensland abattoirs. Epidemiol Infect 104:267-273.

207. Fries LF, Waag DM, Williams JC. 1993. Safety and immunogenicity in human volunteers of a chloroform-methanol residue vaccine for $Q$ fever. Infect Immun 61:1251-1258.

208. Parker N, Robson J, Bell M. 2010. A serosurvey of Coxiella burnetii infection in children and young adults in South West Queensland. Aust N Z J Public Health 34:79-82.

209. Barralet JH, Parker NR. 2004. Q fever in children: an emerging public health issue in Queensland. Med J Aust 180:596-597.

210. Wiedermann U, Garner-Spitzer E, Wagner A. 2016. Primary vaccine failure to routine vaccines: why and what to do? Hum Vaccin Immunother 12:239243. 
211. McBrien CN, Menzies-Gow A. 2017. The biology of eosinophils and their role in asthma. Front Med 4:93.

212. O'Sullivan JA, Bochner BS. 2018. Eosinophils and eosinophil-associated diseases: an update. J Allergy Clin Immunol 141:505-517.

213. Weller PF, Spencer LA. 2017. Functions of tissue-resident eosinophils. Nat Rev Immunol 17:746-760.

214. Rosenberg HF, Dyer KD, Foster PS. 2013. Eosinophils: changing perspectives in health and disease. Nat Rev Immunol 13:9-22.

215. Haskell MD, Moy JN, Gleich GJ, Thomas LL. 1995. Analysis of signaling events associated with activation of neutrophil superoxide anion production by eosinophil granule major basic protein. Blood 86:4627-4637.

216. Elliott A, Schoenlaub L, Freches D, Mitchell W, Zhang G. 2015. Neutrophils play an important role in protective immunity against Coxiella burnetii infection. Infect Immun 83:3104-3113.

217. Yang D, Rosenberg HF, Chen Q, Dyer KD, Kurosaka K, Oppenheim JJ. 2003. Eosinophil-derived neurotoxin (EDN), an antimicrobial protein with chemotactic activities for dendritic cells. Blood 102:3396-3403.

218. Yang D, Chen Q, Su SB, Zhang P, Kurosaka K, Caspi RR, Michalek SM, Rosenberg HF, Zhang N, Oppenheim JJ. 2008. Eosinophil-derived neurotoxin acts as an alarmin to activate the TLR2-MyD88 signal pathway in dendritic cells and enhances Th2 immune responses. J Exp Med 205:7990. 
219. Weller PF, Rand TH, Barrett T, Elovic A, Wong DT, Finberg RW. 1993. Accessory cell function of human eosinophils. HLA-DR-dependent, MHCrestricted antigen-presentation and IL-1 alpha expression. J Immunol 150:2554-2562.

220. Wang HB, Weller PF. 2008. Pivotal advance: eosinophils mediate early alum adjuvant-elicited B cell priming and IgM production. J Leukoc Biol 83:817-821.

221. Rankin JA, Harris P, Ackerman SJ. 1992. The effects of eosinophil-granule major basic protein on lung-macrophage superoxide anion generation. $\mathrm{J}$ Allergy Clin Immunol 89:746-752.

222. Akporiaye ET, Baca OG. 1983. Superoxide anion production and superoxide dismutase and catalase activities in Coxiella burnetii. J Bacteriol 154:520-523.

223. Akporiaye ET, Stefanovich D, Tsosie V, Baca G. 1990. Coxiella burnetii fails to stimulate human neutrophil superoxide anion production. Acta Virol 34:64-70.

224. Baca OG, Roman MJ, Glew RH, Christner RF, Buhler JE, Aragon AS. 1993. Acid phosphatase activity in Coxiella burnetii: a possible virulence factor. Infect Immun 61:4232-4239.

225. Hill J, Samuel JE. 2011. Coxiella burnetii acid phosphatase inhibits the release of reactive oxygen intermediates in polymorphonuclear leukocytes. Infect Immun 79:414-420. 
226. Domachowske JB, Dyer KD, Bonville CA, Rosenberg HF. 1998. Recombinant human eosinophil-derived neurotoxin/RNase 2 functions as an effective antiviral agent against respiratory syncytial virus. J Infect Dis $177: 1458-1464$.

227. Rosenberg HF, Domachowske JB. 2001. Eosinophils, eosinophil ribonucleases, and their role in host defense against respiratory virus pathogens. J Leukoc Biol 70:691-698.

228. Phipps S, Lam CE, Mahalingam S, Newhouse M, Ramirez R, Rosenberg HF, Foster PS, Matthaei KI. 2007. Eosinophils contribute to innate antiviral immunity and promote clearance of respiratory syncytial virus. Blood 110:1578-1586.

229. Davoine F, Cao M, Wu Y, Ajamian F, Ilarraza R, Kokaji Al, Moqbel R, Adamko DJ. 2008. Virus-induced eosinophil mediator release requires antigen-presenting and CD4+ T cells. J Allergy Clin Immunol 122:69-77.e2.

230. Percopo CM, Dyer KD, Ochkur SI, Luo JL, Fischer ER, Lee JJ, Lee NA, Domachowske JB, Rosenberg HF. 2014. Activated mouse eosinophils protect against lethal respiratory virus infection. Blood 123:743-752.

231. Flores-Torres AS, Salinas-Carmona MC, Salinas E, Rosas-Taraco AG. 2019. Eosinophils and respiratory viruses. Viral Immunol 32:198-207.

232. Borelli V, Vita F, Shankar S, Soranzo MR, Banfi E, Scialino G, Brochetta C, Zabucchi G. 2003. Human eosinophil peroxidase induces surface 
alteration, killing, and lysis of Mycobacterium tuberculosis. Infect Immun $71: 605-613$.

233. Driss V, Legrand F, Hermann E, Loiseau S, Guerardel Y, Kremer L, Adam E, Woerly G, Dombrowicz D, Capron M. 2009. TLR2-dependent eosinophil interactions with mycobacteria: role of alpha-defensins. Blood 113:32353244.

234. Shi HZ, Humbles A, Gerard C, Jin Z, Weller PF. 2000. Lymph node trafficking and antigen presentation by endobronchial eosinophils. J Clin Invest 105:945-953.

235. Spencer LA, Szela CT, Perez SA, Kirchhoffer CL, Neves JS, Radke AL, Weller PF. 2009. Human eosinophils constitutively express multiple Th1, Th2, and immunoregulatory cytokines that are secreted rapidly and differentially. J Leukoc Biol 85:117-123.

236. Wong TW, Doyle AD, Lee JJ, Jelinek DF. 2014. Eosinophils regulate peripheral B cell numbers in both mice and humans. J Immunol 192:35483558.

237. Chu VT, Frohlich A, Steinhauser G, Scheel T, Roch T, Fillatreau S, Lee JJ, Lohning M, Berek C. 2011. Eosinophils are required for the maintenance of plasma cells in the bone marrow. Nat Immunol 12:151-159.

238. Chu VT, Berek C. 2012. Immunization induces activation of bone marrow eosinophils required for plasma cell survival. Eur J Immunol 42:130-137. 
239. Chu VT, Berek C. 2013. The establishment of the plasma cell survival niche in the bone marrow. Immunol Rev 251:177-188.

240. Banchereau J, Briere F, Caux C, Davoust J, Lebecque S, Liu YJ, Pulendran B, Palucka K. 2000. Immunobiology of dendritic cells. Annu Rev Immunol 18:767-811.

241. Mosser DM. 2003. The many faces of macrophage activation. J Leukoc Biol 73:209-212.

242. Igietseme JU, Eko FO, He Q, Black CM. 2003. Antibody regulation of T-cell immunity: implications for vaccine strategies against intracellular pathogens. Expert Rev Vaccines 3:23-34.

243. Tangye SG, Hodgkin PD. 2004. Divide and conquer: the importance of cell division in regulating B-cell responses. Immunology 112:509-520.

244. Schroder K, Hertzog PJ, Ravasi T, Hume DA. 2004. Interferon-gamma: an overview of signals, mechanisms and functions. J Leukoc Biol 75:163-189.

245. Turco J, Thompson HA, Winkler HH. 1984. Interferon-gamma inhibits growth of Coxiella burnetii in mouse fibroblasts. Infect Immun 45:781-783.

246. Dellacasagrande J, Capo C, Raoult D, Mege JL. 1999. IFN-gammamediated control of Coxiella burnetii survival in monocytes: the role of cell apoptosis and TNF. J Immunol 162:2259-2265.

247. Howe D, Barrows LF, Lindstrom NM, Heinzen RA. 2002. Nitric oxide inhibits Coxiella burnetii replication and parasitophorous vacuole maturation. Infect Immun 70:5140-5147. 
248. Andoh M, Russell-Lodrigue KE, Zhang G, Samuel JE. 2005. Comparative virulence of phase I and II Coxiella burnetii in immunodeficient mice. Ann N Y Acad Sci 1063:167-170.

249. Crotty S, Johnston RJ, Schoenberger SP. 2010. Effectors and memories: Bcl-6 and Blimp-1 in T and B lymphocyte differentiation. Nat Immunol $11: 114-120$.

250. Ghiasi H, Cai S, Nesburn AB, Wechsler SL. 1997. MHC-II but not MHC-I responses are required for vaccine-induced protection against ocular challenge with HSV-1. Curr Eye Res 16:1152-1158.

251. Ghiasi H, Roopenian DC, Slanina S, Cai S, Nesburn AB, Wechsler SL. 1997. The importance of MHC-I and MHC-II responses in vaccine efficacy against lethal herpes simplex virus type 1 challenge. Immunology 91:430435.

252. O E, Lee YT, Ko EJ, Kim KH, Lee YN, Song JM, Kwon YM, Kim MC, Perez DR, Kang SM. 2014. Roles of major histocompatibility complex class II in inducing protective immune responses to influenza vaccination. J Virol 88:7764-7775.

253. Ladel CH, Daugelat S, Kaufmann SHE. 1995. Immune response to Mycobacterium bovis bacille Calmette Guérin infection in major histocompatibility complex class $\mathrm{I}$ - and II-deficient knock-out mice: contribution of CD4 and CD8 T cells to acquired resistance. Eur J Immunol doi:10.1002/eji.1830250211:377-384. 
254. Praetor A, Hunziker W. 2002. $\beta 2$-microglobulin is important for cell surface expression and $\mathrm{pH}$-dependent IgG binding of human FcRn. J Cell Sci 115:2389-2397.

255. Ghetie V, Hubbard JG, Kim J-K, Tsen M-F, Lee Y, Ward ES. 1996. Abnormally short serum half-lives of IgG in $\beta 2$-microglobulin-deficient mice. Eur J Immunol 26:690-696.

256. Israel EJ, Wilsker DF, Hayes KC, Schoenfeld D, Simister NE. 1996. Increased clearance of $\lg G$ in mice that lack $\beta 2$-microglobulin: possible protective role of FcRn. Immunology 89:573-578.

257. Christianson GJ, Brooks W, Vekasi S, Manolfi EA, Niles J, Roopenian SL, Roths JB, Rothlein R, Roopenian DC. 1997. ß2-microglobulin-deficient mice are protected from hypergammaglobulinemia and have defective antibody responses because of increased IgG catabolism. J Immunol 159:4781-4792.

258. Spriggs MK, Koller BH, Sato T, Morrissey PJ, Fanslow WC, Smithies O, Voice RF, Widmer MB, Maliszewski CR. 1992. ß2-microglobulin-, CD8+ Tcell-deficient mice survive inoculation with high doses of vaccinia virus and exhibit altered IgG responses. Proc Natl Acad Sci 89:6070-6074.

259. Yang R, Masters AR, Fortner KA, Champagne DP, Yanguas-Casás N, Silberger DJ, Weaver CT, Haynes L, Rincon M. 2016. IL-6 promotes the differentiation of a subset of naive CD8+ T cells into IL-21-producing $B$ helper CD8+ T cells. J Exp Med 213:2281-2291. 
260. Locksley RM, Reiner SL, Hatam F, Littman DR, Killeen N. 1993. Helper T cells without CD4: control of Leishmaniasis in CD4-deficient mice. Science 261:1448-1451.

261. Rahemtulla A, Kundig TM, Narendran A, Bachmann MF, Julius M, Paige CJ, Ohashi PS, Zinkernagel RM, Mak TW. 1994. Class II major histocompatibility complex-restricted T cell function in CD4-deficient mice. Eur J Immunol 24:2213-2218.

262. Matechak EO, Killeen N, Hedrick SM, Fowlkes BJ. 1996. MHC class IIspecific $\mathrm{T}$ cells can develop in the CD8 lineage when CD4 is absent. Immunity 4:337-347.

263. Pearce EL, Shedlock DJ, Shen H. 2004. Functional characterization of MHC class II-restricted CD8+CD4- and CD8-CD4- T cell responses to infection in CD4-/- mice. J Immunol 173:2494-2499.

264. Tyznik AJ, Sun JC, Bevan MJ. 2004. The CD8 population in CD4-deficient mice is heavily contaminated with MHC class II-restricted T cells. J Exp Med 199:559-565

265. Zheng B, Ozen ZZ, Cao S, Zhang Y, Han S. 2002. CD4-deficient T helper cells are capable of supporting somatic hypermutation and affinity maturation of germinal center B cells. Eur J Immunol 32:3315-3325.

266. Schneider T, Jahn HU, Liesenfeld O, Steinhoff D, Riecken EO, Zeitz M, Ullrich R. 1997. The number and proportion of Vgamma9 Vdelta2 T cells 
rise significantly in the peripheral blood of patients after the onset of acute Coxiella burnetii infection. Clin Infect Dis 24:261-264.

267. Szabo SJ, Kim ST, Costa GL, Zhang X, Fathman CG, Glimcher LH. 2000. A novel transcription factor, T-bet, directs Th1 lineage commitment. Cell 100:655-669.

268. Zheng W, Flavell RA. 1997. The transcription factor GATA-3 is necessary and sufficient for Th2 cytokine gene expression in CD4 T cells. Cell 89:587596.

269. Zhu J, Guo L, Watson CJ, Hu-Li J, Paul WE. 2001. Stat6 is necessary and sufficient for IL-4's role in Th2 differentiation and cell expansion. J Immunol 166:7276-7281.

270. Harrington LE, Hatton RD, Mangan PR, Turner H, Murphy TL, Murphy KM, Weaver CT. 2005. Interleukin 17-producing CD4+ effector T cells develop via a lineage distinct from the T helper type 1 and 2 lineages. Nat Immunol 6:1123-1132.

271. Lugo-Villarino G, Maldonado-Lopez R, Possemato R, Penaranda C, Glimcher LH. 2003. T-bet is required for optimal production of IFN-gamma and antigen-specific T cell activation by dendritic cells. Proc Natl Acad Sci 100:7749-7754.

272. Peng SL, Szabo SJ, Glimcher LH. 2002. T-bet regulates IgG class switching and pathogenic autoantibody production. Proc Natl Acad Sci 99:5545-5550. 
273. Gerth AJ, Lin L, Peng SL. 2003. T-bet regulates T-independent IgG2a class switching. Int Immunol 15:937-944.

274. Sullivan BM, Juedes A, Szabo SJ, von Herrath M, Glimcher LH. 2003. Antigen-driven effector CD8 T cell function regulated by T-bet. Proc Natl Acad Sci 100:15818-15823.

275. Intlekofer AM, Takemoto N, Wherry EJ, Longworth SA, Northrup JT, Palanivel VR, Mullen AC, Gasink CR, Kaech SM, Miller JD, Gapin L, Ryan K, Russ AP, Lindsten T, Orange JS, Goldrath AW, Ahmed R, Reiner SL. 2005. Effector and memory CD8+ $\mathrm{T}$ cell fate coupled by $\mathrm{T}$-bet and eomesodermin. Nat Immunol 6:1236-1244.

276. Joshi NS, Cui W, Chandele A, Lee HK, Urso DR, Hagman J, Gapin L, Kaech SM. 2007. Inflammation directs memory precursor and short-lived effector CD8(+) T cell fates via the graded expression of T-bet transcription factor. Immunity 27:281-295.

277. Sullivan BM, Jobe O, Lazarevic V, Vasquez K, Bronson R, Glimcher LH, Kramnik I. 2005. Increased susceptibility of mice lacking T-bet to infection with Mycobacterium tuberculosis correlates with increased $\mathrm{IL}-10$ and decreased IFN-gamma production. J Immunol 175:4593-4602.

278. Ravindran R, Foley J, Stoklasek T, Glimcher LH, McSorley SJ. 2005. Expression of T-bet by CD4 T cells is essential for resistance to Salmonella infection. J Immunol 175:4603-4610. 
279. Usui T, Preiss JC, Kanno Y, Yao ZJ, Bream JH, O'Shea JJ, Strober W. 2006. T-bet regulates Th1 responses through essential effects on GATA-3 function rather than on IFNG gene acetylation and transcription. J Exp Med 203:755-766.

280. Gidding HF, Wallace C, Lawrence GL, McIntyre PB. 2009. Australia's national Q fever vaccination program. Vaccine 27:2037-2041.

281. Ghigo E, Capo C, Tung CH, Raoult D, Gorvel JP, Mege JL. 2002. Coxiella burnetii survival in THP-1 monocytes involves the impairment of phagosome maturation: IFN-gamma mediates its restoration and bacterial killing. J Immunol 169:4488-4495.

282. Dellacasagrande J, Ghigo E, Raoult D, Capo C, Mege JL. 2002. IFNgamma-induced apoptosis and microbicidal activity in monocytes harboring the intracellular bacterium Coxiella burnetii require membrane TNF and homotypic cell adherence. J Immunol 169:6309-6315.

283. Thakur A, Mikkelsen H, Jungersen G. 2019. Intracellular pathogens: host immunity and microbial persistence strategies. J Immunol Res 2019:1-24.

284. Jerrells TR, Mallavia LP, Hinrichs DJ. 1975. Detection of long-term cellular immunity to Coxiella burnetii as assayed by lymphocyte transformation. Infect Immun 11:280-286.

285. Ascher MS, Berman MA, Parker D, Turk JL. 1983. Experimental model for dermal granulomatous hypersensitivity in $Q$ fever. Infect Immun 39:388393. 
286. Kazar J, Votruba D, Propper P, Schramek S. 1986. Onset and duration of immunity in guinea pigs and mice induced with different $Q$ fever vaccines. Acta Virol 30:499-506.

287. Ruble DL, Elliott JJ, Waag DM, Jaax GP. 1994. A refined guinea pig model for evaluating delayed-type hypersensitivity reactions caused by $Q$ fever vaccines. Lab Anim Sci 44:608-612.

288. Wilhelmsen CL, Waag DM. 2000. Guinea pig abscess/hypersensitivity model for study of adverse vaccination reactions induced by use of $Q$ fever vaccines. Comp Med 50:374-378.

289. Izzo AA, Marmion BP, Hackstadt T. 1991. Analysis of the cells involved in the lymphoproliferative response to Coxiella burnetii antigens. Clin Exp Immunol 85:98-108.

290. Mou Z, Liu D, Okwor I, Jia P, Orihara K, Uzonna JE. 2014. MHC class II restricted innate-like double negative $\mathrm{T}$ cells contribute to optimal primary and secondary immunity to Leishmania major. PLoS Pathog 10:e1004396.

291. Mata-Haro V, Cekic C, Martin M, Chilton PM, Casella CR, Mitchell TC. 2007. The vaccine adjuvant monophosphoryl lipid A as a TRIF-biased agonist of TLR4. Science 316:1628-1632.

292. Coffman RL, Sher A, Seder RA. 2010. Vaccine adjuvants: putting innate immunity to work. Immunity 33:492-503.

293. Pulendran B. 2004. Modulating vaccine responses with dendritic cells and Toll-like receptors. Immunol Rev 199:227-250. 
294. Pulendran B, Smith JL, Caspary G, Brasel K, Pettit D, Maraskovsky E, Maliszewski CR. 1999. Distinct dendritic cell subsets differentially regulate the class of immune response in vivo. Proc Natl Acad Sci 96:1036-1041.

295. Maldonado-Lopez R, De Smedt T, Michel P, Godfroid J, Pajak B, Heirman C, Thielemans K, Leo O, Urbain J, Moser M. 1999. CD8alphat and CD8alpha- subclasses of dendritic cells direct the development of distinct T helper cells in vivo. J Exp Med 189:587-592.

296. Rissoan MC, Soumelis V, Kadowaki N, Grouard G, Briere F, de Waal Malefyt R, Liu YJ. 1999. Reciprocal control of T helper cell and dendritic cell differentiation. Science 283:1183-1186.

297. Agrawal S, Agrawal A, Doughty B, Gerwitz A, Blenis J, Van Dyke T, Pulendran B. 2003. Cutting edge: different Toll-like receptor agonists instruct dendritic cells to induce distinct Th responses via differential modulation of extracellular signal-regulated kinase-mitogen-activated protein kinase and c-Fos. J Immunol 171:4984-9.

298. Dillon S, Agrawal A, Van Dyke T, Landreth G, McCauley L, Koh A, Maliszewski C, Akira S, Pulendran B. 2004. A Toll-like receptor 2 ligand stimulates Th2 responses in vivo, via induction of extracellular signalregulated kinase mitogen-activated protein kinase and c-Fos in dendritic cells. J Immunol 172:4733-4743.

299. Zamboni DS, Campos MA, Torrecilhas AC, Kiss K, Samuel JE, Golenbock DT, Lauw FN, Roy CR, Almeida IC, Gazzinelli RT. 2004. Stimulation of Toll- 
like receptor 2 by Coxiella burnetii is required for macrophage production of pro-inflammatory cytokines and resistance to infection. J Biol Chem 279:54405-54415.

300. Meghari S, Honstettre A, Lepidi H, Ryffel B, Raoult D, Mege JL. 2005. TLR2 is necessary to inflammatory response in Coxiella burnetii infection. Ann N Y Acad Sci 1063:161-166.

301. Ammerdorffer A, Schoffelen T, Gresnigt MS, Oosting M, den Brok MH, Abdollahi-Roodsaz S, Kanneganti TD, de Jong DJ, van Deuren M, Roest HJ, Rebel JM, Netea MG, Joosten LA, Sprong T. 2015. Recognition of Coxiella burnetii by toll-like receptors and nucleotide-binding oligomerization domain-like receptors. J Infect Dis 211:978-87.

302. Ramstead AG, Robison A, Blackwell A, Jerome M, Freedman B, Lubick KJ, Hedges JF, Jutila MA. 2016. Roles of Toll-like receptor 2 (TLR2), TLR4, and MyD88 during pulmonary Coxiella burnetii infection. Infect Immun 84:940949.

303. Mbow ML, De Gregorio E, Valiante NM, Rappuoli R. 2010. New adjuvants for human vaccines. Curr Opin Immunol 22:411-416.

304. Dupuis M, Murphy TJ, Higgins D, Ugozzoli M, van Nest G, Ott G, McDonald DM. 1998. Dendritic cells internalize vaccine adjuvant after intramuscular injection. Cell Immunol 186:18-27. 
305. Mosca F, Tritto E, Muzzi A, Monaci E, Bagnoli F, lavarone C, O'Hagan D, Rappuoli R, De Gregorio E. 2008. Molecular and cellular signatures of human vaccine adjuvants. Proc Natl Acad Sci 105:10501-10506.

306. Banzhoff A, Gasparini R, Laghi-Pasini F, Staniscia T, Durando P, Montomoli E, Capecchi PL, di Giovanni P, Sticchi L, Gentile C, Hilbert A, Brauer V, Tilman S, Podda A. 2009. MF59-adjuvanted H5N1 vaccine induces immunologic memory and heterotypic antibody responses in non-elderly and elderly adults. PLoS One 4:e4384.

307. Vesikari T, Groth N, Karvonen A, Borkowski A, Pellegrini M. 2009. MF59adjuvanted influenza vaccine (FLUAD) in children: safety and immunogenicity following a second year seasonal vaccination. Vaccine 27:6291-6295. 


\section{VITA}

Lindsey E. Ledbetter was born to Alan and Jeri Pritchett on November 16, 1992, in Little Rock, Arkansas. Lindsey is the younger half-sister of Laura Keeter and the late James Pritchett, and the older sister of Katie Pritchett. She spent her early childhood in Benton, Arkansas and moved to Harrison, Arkansas in 2002. Lindsey graduated from Bergman High School in 2011 as valedictorian with distinguished highest honors. She earned her Bachelor of Science in Agricultural, Food, and Life Sciences in 2014 from the University of Arkansas, where she graduated Magna Cum Laude with honors. Lindsey joined the Molecular Pathogenesis and Therapeutics program at the University of Missouri in June 2014 under the supervision of Dr. Guoquan Zhang. She earned her Doctor of Philosophy in Molecular Microbiology and Immunology in December 2019. 